\title{
Distances in random graphs with finite mean and infinite variance degrees
}

\author{
Remco van der Hofstad* \\ Gerard Hooghiemstra ${ }^{\dagger}$ and Dmitri Znamenski ${ }^{\ddagger}$
}

June 23, 2021

\begin{abstract}
In this paper we study random graphs with independent and identically distributed degrees of which the tail of the distribution function is regularly varying with exponent $\tau \in(2,3)$.

The number of edges between two arbitrary nodes, also called the graph distance or hopcount, in a graph with $N$ nodes is investigated when $N \rightarrow \infty$. When $\tau \in(2,3)$, this graph distance grows like $2 \frac{\log \log N}{|\log (\tau-2)|}$. In different papers, the cases $\tau>3$ and $\tau \in(1,2)$ have been studied. We also study the fluctuations around these asymptotic means, and describe their distributions. The results presented here improve upon results of Reittu and Norros, who prove an upper bound only.
\end{abstract}

AMS 1991 subject classifications. Primary 05C80; secondary 60J80.

Key words and phrases. Configuration model, graph distance.

\section{Introduction}

The study of complex networks plays an increasingly important role in science. Examples of complex networks are electrical power grids and telephony networks, social relations, the World-Wide Web and Internet, co-authorship and citation networks of scientists, etc. The structure of networks affects their performance and function. For instance, the topology of social networks affects the spread of information and infections. Measurements on complex networks have shown that many networks have similar properties. A first key example of such a fundamental network property is the fact that typical distances between nodes are small, which is called the 'small world' phenomenon. A second key example shared by many networks is that the number of nodes with degree $k$ falls off as an inverse power of $k$, which is called a power law degree sequence. See [4, 29, 36] and the references therein for an introduction to complex networks and many examples where the above two properties hold.

The current paper presents a rigorous derivation for the random fluctuations of the graph distance between two arbitrary nodes (also called the geodesic, and in Internet called the hopcount) in a graph with infinite variance degrees. The model studied here is a variant of the configuration model. The infinite variance degrees include power laws with exponent $\tau \in(2,3)$. In practice, power exponents are observed ranging between $\tau=1.5$ and $\tau=3.2$ (see [29]).

In a previous paper of the first two authors with Van Mieghem [21], we investigated the finite variance case $\tau>3$. In [22], we study the case where $\tau \in(1,2)$. Apart from the critical cases $\tau=2$

\footnotetext{
* Department of Mathematics and Computer Science, Eindhoven University of Technology, P.O. Box 513, 5600 MB Eindhoven, The Netherlands. E-mail: rhofstad@win.tue.nl

${ }^{\dagger}$ Delft University of Technology, Electrical Engineering, Mathematics and Computer Science, P.O. Box 5031, 2600 GA Delft, The Netherlands. E-mail: G.Hooghiemstra@ewi.tudelft.nl

${ }^{\ddagger}$ EURANDOM, P.O. Box 513, 5600 MB Eindhoven, The Netherlands. E-mail: znamenski@eurandom.nl
} 
and $\tau=3$, we have thus investigated all possible values of $\tau$. The paper 23] serves as a survey to the results and, in particular, describes how our results can be applied to Internet data, describes related work on random graphs that are similar, though not identical to ours, and gives further open problems. Finally, in [23], we also investigate the structure of the connected components in the random graphs under consideration. See [5, 6, 25] for an introduction to classical random graphs.

This section is organised as follows. In Section 1.1 we start by introducing the model, in Section 1.2 we state our main results. Section 1.3 is devoted to related work, and in Section 1.4 we describe some simulations for a better understanding of the results.

\section{$1.1 \quad$ Model definition}

Fix an integer $N$. Consider an i.i.d. sequence $D_{1}, D_{2}, \ldots, D_{N}$. We will construct an undirected graph with $N$ nodes where node $j$ has degree $D_{j}$. We will assume that $L_{N}=\sum_{j=1}^{N} D_{j}$ is even. If $L_{N}$ is odd, then we increase $D_{N}$ is by 1 . This change will make hardly any difference in what follows, and we will ignore this effect. We will later specify the distribution of $D_{1}$.

To construct the graph, we have $N$ separate nodes and incident to node $j$, we have $D_{j}$ stubs. All stubs need to be connected to another stub to build the graph. The stubs are numbered in an arbitrary order from 1 to $L_{N}$. We start by connecting at random the first stub with one of the $L_{N}-1$ remaining stubs. Once paired, two stubs form a single edge of the graph. We continue the procedure of randomly choosing and pairing the stubs until all stubs are connected. Unfortunately, nodes having self-loops may occur. However, self-loops are scarce when $N \rightarrow \infty$.

The above model is a variant of the configuration model, which, given a degree sequence, is the random graph with that given degree sequence. For a graph, the degree sequence of that graph is the vectors of which the $k^{\text {th }}$ coordinate equals the frequency of nodes with degree $k$. In our model, the degree sequence is very close to the distribution of the nodal degree $D$ of which $D_{1}, \ldots, D_{N}$ are i.i.d. copies. The probability mass function and the distribution function of the nodal degree law are denoted by

$$
\mathbb{P}\left(D_{1}=j\right)=f_{j}, \quad j=1,2, \ldots, \quad \text { and } \quad F(x)=\sum_{j=1}^{\lfloor x\rfloor} f_{j},
$$

where $\lfloor x\rfloor$ is the largest integer smaller than or equal to $x$. Our main assumption is that we take

$$
1-F(x)=x^{-\tau+1} L(x),
$$

where $\tau \in(2,3)$ and $L$ is slowly varying at infinity. This means that the random variables $D_{i}$ obey

a power law, and the factor $L$ is meant to generalize the model. We work under a slightly more restrictive assumption:

Assumption 1.1 There exists $\gamma \in[0,1)$ and $C>0$ such that

$$
x^{-\tau+1-C(\log x)^{\gamma-1}} \leq 1-F(x) \leq x^{-\tau+1+C(\log x)^{\gamma-1}}, \quad \text { for large } x .
$$

Comparing with (1.2), we see that the slowly varying function $L$ in (1.2) should satisfy

$$
e^{-C(\log x)^{\gamma}} \leq L(x) \leq e^{C(\log x)^{\gamma}} .
$$

\subsection{Main results}

We define the graph distance $H_{N}$ between the nodes 1 and 2 as the minimum number of edges that form a path from 1 to 2 . By convention, the distance equals $\infty$ if 1 and 2 are not connected. Observe that the distance between two randomly chosen nodes is equal in distribution to $H_{N}$, because the nodes are exchangeable. We now describe our main result. 
Theorem 1.2 (Fluctuations of the Graph Distance) Assume that Assumption [1.1 holds and fix $\tau \in(2,3)$ in (1.2). Then there exist random variables $\left(R_{a}\right)_{a \in(-1,0]}$ such that, as $N \rightarrow \infty$,

$$
\mathbb{P}\left(H_{N}=2\left\lfloor\frac{\log \log N}{|\log (\tau-2)|}\right\rfloor+l \mid H_{N}<\infty\right)=\mathbb{P}\left(R_{a_{N}}=l\right)+o(1), \quad l \in \mathbb{Z},
$$

where $a_{N}=\left\lfloor\frac{\log \log N}{|\log (\tau-2)|}\right\rfloor-\frac{\log \log N}{|\log (\tau-2)|} \in(-1,0]$.

In words, Theorem 1.2 states that for $\tau \in(2,3)$, the graph distance $H_{N}$ between two randomly chosen connected nodes grows proportional to log log of the size of the graph, and that the fluctuations around this mean remain uniformly bounded in $N$.

We identify the laws of $\left(R_{a}\right)_{a \in(-1,0]}$ below. Before doing so, we state two consequences of the above theorem:

Corollary 1.3 (Convergence in Distribution along Subsequences) Along the sequence $N_{k}=$ $\left\lfloor N_{1}^{(\tau-2)^{-(k-1)}}\right\rfloor$, where $k=1,2, \ldots$, and conditionally on 1 and 2 being connected, the random variables

$$
H_{N_{k}}-2\left\lfloor\frac{\log \log N_{k}}{|\log (\tau-2)|}\right\rfloor
$$

converge in distribution to $R_{a_{N_{1}}}$, as $k \rightarrow \infty$.

Simulations illustrating the weak convergence in Corollary 1.3 are discussed in Section 1.4. In the corollary below, we write that an event $E$ occurs whp for the statement that $\mathbb{P}(E)=1-o(1)$.

\section{Corollary 1.4 (Concentration of the Graph Distance)}

(i) Conditionally on 1 and 2 being connected, the random variable $H_{N}$ is, whp, in between $2 \frac{\log \log N}{|\log (\tau-2)|}(1 \pm \varepsilon)$, for any $\varepsilon>0$.

(ii) Conditionally on 1 and 2 being connected, the random variables $H_{N}-\frac{\log \log N}{|\log (\tau-2)|}$ form a tight sequence, i.e.,

$$
\lim _{K \rightarrow \infty} \limsup _{N \rightarrow \infty} \mathbb{P}\left(\left|H_{N}-2 \frac{\log \log N}{|\log (\tau-2)|}\right| \leq K \mid H_{N}<\infty\right)=1 .
$$

We need a limit result from branching processes theory before we can identify the limiting random variables $\left(R_{a}\right)_{a \in(-1,0]}$. In Section 2 we introduce a delayed branching process $\left\{\mathcal{Z}_{k}\right\}_{k \geq 1}$, where in the first generation the offspring distribution is chosen according to (1.1) and in the second and further generations the offspring is chosen in accordance to $g$ given by

$$
g_{j}=\frac{(j+1) f_{j+1}}{\mu}, \quad j=0,1, \ldots,
$$

where $\mu=\sum_{j=1}^{\infty} j f_{j}$. The branching process $\left\{\mathcal{Z}_{k}\right\}$ has infinite expectation. Branching processes with infinite expectation have been investigated in [16, 34, 33. Assumption 1.1, using the results in [16, implies that

$$
(\tau-2)^{n} \cdot \log \left(\mathcal{Z}_{n} \vee 1\right) \rightarrow Y, \quad \text { a.s., }
$$

where $x \vee y=\max \{x, y\}$. See Section 2 and the references there for more details. Then, we can identify the law of the random variables $\left(R_{a}\right)_{a \in(-1,0]}$ as follows:

Theorem 1.5 (The Limit Laws) For $a \in(-1,0]$,

$$
\mathbb{P}\left(R_{a}>l\right)=\mathbb{P}\left(\min _{s \in \mathbb{Z}}\left[(\tau-2)^{-s} Y^{(1)}+(\tau-2)^{s-c_{l}} Y^{(2)}\right] \leq(\tau-2)^{\lceil l / 2\rceil+a} \mid Y^{(1)} Y^{(2)}>0\right),
$$

where $c_{l}=1$ if $l$ is even, and zero otherwise, and $Y^{(1)}, Y^{(2)}$ are two independent copies of the limit random variable in (1.9). 
In Remarks 4.1 and A.1.5 below, we will explain that our results also apply to the usual configuration model, where the number of nodes with a given degree is fixed, when we study the graph distance between two uniformly chosen nodes, and the degree distribution satisfied certain conditions. For the precise conditions, see Remark A.1.5.

\subsection{Related work}

There is a wealth of related work which we now summarize. The model investigated here was also studied in [32, with $1-F(x)=x^{-\tau+1} L(x)$, where $\tau \in(2,3)$ and $L$ denotes a slowly varying function. It was shown in 32 that whp the graph distance is bounded from above by $2 \frac{\log \log N}{|\log (\tau-2)|}(1+o(1))$. We improve the results in 32 by deriving the asymptotic distribution of the random fluctuations of the graph distance around $2\left\lfloor\frac{\log \log N}{\log (\tau-2)\rfloor}\right\rfloor$. Note that these results are in contrast to [30, Section II.F, below (56)], where it was suggested that if $\tau<3$, then an exponential cut-off is necessary to make the graph distance between an arbitrary pair of nodes well-defined. The problem of the graph distance between an arbitrary pair of nodes was also studied non-rigorously in [14, where also the behavior when $\tau=3$ and $x \mapsto L(x)$ is the constant function, is included. In the latter case, the graph distance scales $\operatorname{like} \frac{\log N}{\log \log N}$. A related model to the one studied here can also be found in [31, where a graph process is defined by adding and removing edges. In [31], the authors prove similar results as in 32 for this related model.

The graph distance for $\tau>3, \tau \in(1,2)$, respectively was treated in two previous publications 21] and 22, respectively. We survey these results together with results on the connected components in [23]. In [23], we also show that when $\tau>2$, the diameter is bounded from below by a constant times $\log N$, which, when $\tau \in(2,3)$ should be contrasted with the average graph distance, which is or order $\log \log N$. Finally, in [23] also the connected components are studied under the condition that $\mu=\mathbb{E}\left[D_{1}\right]>2$, and the results in this paper are used to show that whp there exists a largest connected component of size $q N[1+o(1)]$, where $q$ is the survival probability of the delayed branching process, while all other connected components are of order at most $\log N$.

There is substantial work on random graphs that are, although different from ours, still similar in spirit. In [1, random graphs were considered with a degree sequence that is precisely equal to a power law, meaning that the number of nodes with degree $k$ is precisely proportional to $k^{-\tau}$. Aiello et al. 1] show that the largest connected component is of the order of the size of the graph when $\tau<\tau_{0}=3.47875 \ldots$, where $\tau_{0}$ is the solution of $\zeta(\tau-2)-2 \zeta(\tau-1)=0$, and where $\zeta$ is the Riemann zeta function. When $\tau>\tau_{0}$, the largest connected component is of smaller order than the size of the graph and more precise bounds are given for the largest connected component. When $\tau \in(1,2)$, the graph is whp connected. The proofs of these facts use couplings with branching processes and strengthen previous results due to Molloy and Reed [27, 28. For this same model, Dorogovtsev et al. [17, 18 investigate the leading asymptotics and the fluctuations around the mean of the graph distance between arbitrary nodes from a theoretical physics point of view, using mainly generating functions.

A second related model can be found in [12, 13], where edges between nodes $i$ and $j$ are present with probability equal to $w_{i} w_{j} / \sum_{l} w_{l}$ for some 'expected degree vector' $w=\left(w_{1}, \ldots, w_{N}\right)$. It is assumed that $\max _{i} w_{i}^{2}<\sum_{i} w_{i}$, so that $w_{i} w_{j} / \sum_{l} w_{l}$ are probabilities. In [12], $w_{i}$ is often taken as $w_{i}=c i^{-\frac{1}{\tau-1}}$, where $c$ is a function of $N$ proportional to $N^{\frac{1}{\tau-1}}$. In this case, the degrees obey a power law with exponent $\tau$. Chung and Lu [12] show that in this case, the graph distance between two uniformly chosen nodes is whp proportional to $\log N(1+o(1))$ when $\tau>3$, and $2 \frac{\log \log N}{|\log (\tau-2)|}(1+o(1))$ when $\tau \in(2,3)$. The difference between this model and ours is that the nodes are not exchangeable in [12, but the observed phenomena are similar. This result can be heuristically understood as follows. Firstly, the actual degree vector in [12] should be close to the expected degree vector. Secondly, for the expected degree vector, we can compute that the number of nodes for which the 


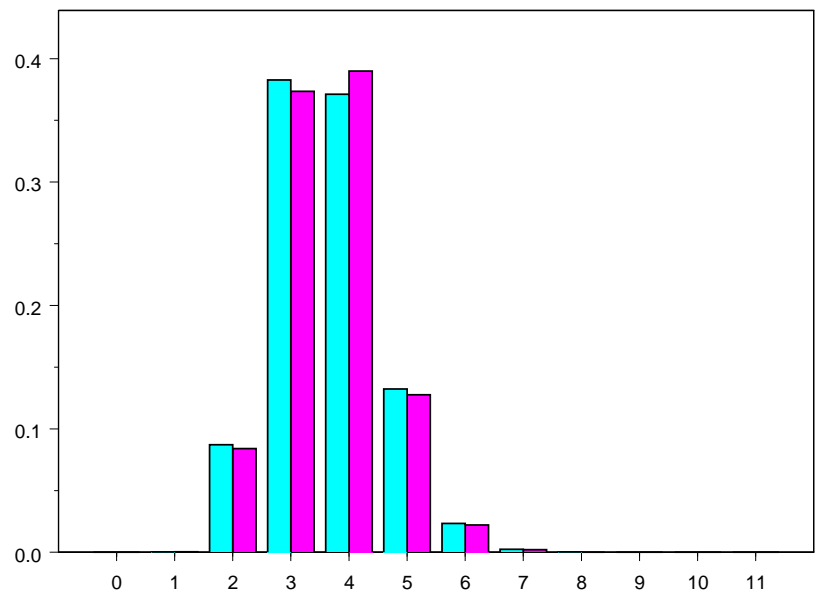

Figure 1: Histograms of the AS-count and graph distance in the configuration model with $N=$ 10, 940, where the degrees have generating function $f_{\tau}(s)$ in (1.11), for which the power law exponent $\tau$ takes the value $\tau=2.25$. The AS-data is lightly shaded, the simulation is darkly shaded.

degree is at least $k$ equals

$$
\left|\left\{i: w_{i} \geq k\right\}\right|=\left|\left\{i: c i^{-\frac{1}{\tau-1}} \geq k\right\}\right| \propto k^{-\tau+1} .
$$

Thus, one expects that the number of nodes with degree at least $k$ decreases as $k^{-\tau+1}$, similarly as in our model. In [13], Chung and Lu study the sizes of the connected components in the above model. The advantage of this model is that the edges are independently present, which makes the resulting graph closer to a traditional random graph.

All the models described above are static, i.e., the size of the graph is fixed, and we have not modeled the growth of the graph. There is a large body of work investigating dynamical models for complex networks, often in the context of the World-Wide Web. In various forms, preferential attachment has been shown to lead to power law degree sequences. Therefore, such models intend to explain the occurrence of power law degree sequences in random graphs. See [2, 3, 4, 7, 8, 9, 10, 11, 15, 26] and the references therein. In the preferential attachment model, nodes with a fixed degree $m$ are added sequentially. Their stubs are attached to a receiving node with a probability proportional to the degree of the receiving node, thus favoring nodes with large degrees. For this model, it is shown that the number of nodes with degree $k$ decays proportionally to $k^{-3}$ [1], the diameter is of order $\frac{\log N}{\log \log N}$ when $m \geq 2$ [8], and couplings to a classical random graph $G(N, p)$ are given for an appropriately chosen $p$ in [10. See also [9] for a survey.

Possibly, the configuration model is a snapshot of the above models, i.e., a realization of the graph growth processes at the time instant that the graph has a certain prescribed size. Thus, rather than to describe the growth of the model, we investigate the properties of the model at a given time instant. This is suggested in [4, Section VII.D], and it would be very interesting indeed to investigate this further mathematically, i.e., to investigate the relation between the configuration and the preferential attachment models.

We study the above version of the configuration model to describe the topology of the Internet at a fixed time instant. In a seminal paper [19], Faloutsos et al. have shown that the degree distribution in Internet follows a power law with exponent $\tau \approx 2.16-2.25$. Thus, the power law random graph with this value of $\tau$ can possibly lead to a good Internet model. In 35, and inspired by the observed power law degree sequence in [19], the power law random graph is proposed as 
a model for the network of autonomous systems. In this graph, the nodes are the autonomous systems in the Internet, i.e., the parts of the Internet controlled by a single party (such as a university, company or provider), and the edges represent the physical connections between the different autonomous systems. The work of Faloutsos et al. in [19] was among others on this graph which at that time had size approximately 10,000.

In [35], it is argued on a qualitative basis that the power law random graph serves as a better model for the Internet topology than the currently used topology generators. Our results can be seen as a step towards the quantitative understanding of whether the AS-count in Internet is described well by the average graph distance in the configuration model. The AS-count gives the number of physical links connecting the various autonomous domains between two randomly chosen nodes in the graph.

To validate the model, we compare a simulation of the distribution of the distance between pairs of nodes in the power law random graph with the same value of $N$ and $\tau$ to extensive measurements of the AS-count in Internet. In Figure 1, we see that AS-count in the model with the predicted value of $\tau=2.25$ and the value of $N$ from the data set fits the data remarkably well.

In [29, Table II], many other examples are given of real networks that have power law degree sequences. Interestingly, there are many examples where the power law exponent is in $(2,3)$, and it would be of interest to compare the average graph distance between an arbitrary pair of nodes in such examples.

\subsection{Demonstration of Corollary 1.3}

By a simulation we explain the relevance of Theorem 1.2 and especially the relevance of Corollary 1.3. We have chosen to simulate the distribution (1.8) from the generating function:

$$
g_{\tau}(s)=1-(1-s)^{\tau-2}, \quad \text { for which } \quad g_{j}=(-1)^{j-1}\left(\begin{array}{c}
\tau-2 \\
j
\end{array}\right) \sim \frac{c}{j^{\tau-1}}, \quad j \rightarrow \infty .
$$

Defining

$$
f_{\tau}(s)=\frac{\tau-1}{\tau-2} s-\frac{1-(1-s)^{\tau-1}}{\tau-2}, \quad \tau \in(2,3)
$$

it is immediate that

$$
g_{\tau}(s)=\frac{f_{\tau}^{\prime}(s)}{f_{\tau}^{\prime}(1)}, \quad \text { so that } \quad g_{j}=\frac{(j+1) f_{j+1}}{\mu} .
$$

For fixed $\tau$, we can pick different values of the size of the simulated graph, so that for each two simulated values $N$ and $M$ we have $a_{N}=a_{M}$, i.e., $N=M^{(\tau-2)^{-k}}$, for some integer $k$. For $\tau=2.8$, we have taken the values

$$
N=1,000, \quad N=5,623, \quad N=48,697, \quad N=723,394 .
$$

According to Theorem 1.2, the survival functions of the hopcount $H_{N}$, satisfying $N=M^{(\tau-2)^{-k}}$, run parallel on distance 2 in the limit for $N \rightarrow \infty$. In Section 2 below we will show that the distribution with generating function (1.11) satisfies Assumption 1.1

\subsection{Organization of the paper}

The paper is organized as follows. We first review the relevant literature on branching processes in Section 2. We then describe the growth of shortest path graphs in Section 3, and we state coupling results needed to prove our main results, Theorems 1.2 1.5 in Section 4. In Section [5 we prove three technical lemmas used in Section 4. We finally prove the coupling results in the Appendix. 


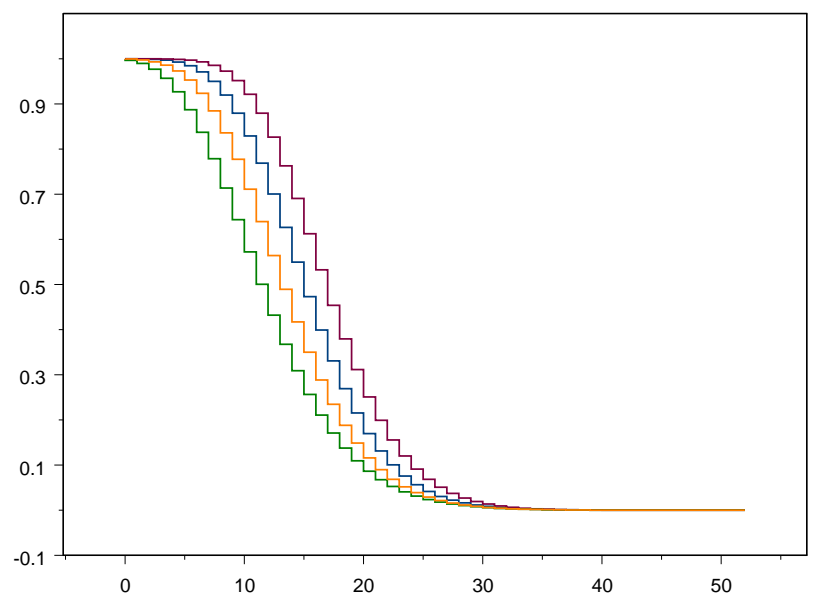

Figure 2: Empirical survival functions of the graph distance for $\tau=2.8$ and for the four values of $N$.

\section{Review of branching process theory with infinite mean}

Since we heavily rely on the theory of branching processes (BP's), we now briefly review this theory in the case where the expected value (mean) of the offspring distribution is infinite. We follow in particular [16, and also refer the readers to related work in [33, 34], and the references therein.

For the formal definition of the BP we define a double sequence $\left\{X_{n, i}\right\}_{n \geq 0, i \geq 1}$ of i.i.d. random variables each with distribution equal to the offspring distribution $\left\{g_{j}\right\}$ given in (1.8) with distribution function $G(x)=\sum_{j=0}^{\lfloor x\rfloor} g_{j}$. The $\mathrm{BP}\left\{\mathcal{Z}_{n}\right\}$ is now defined by $\mathcal{Z}_{0}=1$ and

$$
\mathcal{Z}_{n+1}=\sum_{i=1}^{\mathcal{Z}_{n}} X_{n, i}, \quad n \geq 0 .
$$

In case of a delayed BP, we let $X_{0,1}$ have probability mass function $\left\{f_{j}\right\}$, independently of $\left\{X_{n, i}\right\}_{n \geq 1, i \geq 1}$. In this section we restrict to the non-delayed case for simplicity.

We follow Davies in [16, who gives the following sufficient conditions for convergence of $(\tau-2)^{n} \log \left(1+\mathcal{Z}_{n}\right)$. Davies' main theorem states that if for some non-negative, non-increasing function $\gamma(x)$ :

(i) $x^{-\alpha-\gamma(x)} \leq 1-G(x) \leq x^{-\alpha+\gamma(x)}, \quad$ for large $x \quad$ and $\quad 0<\alpha<1$,

(ii) $x^{\gamma(x)}$ is non-decreasing,

(iii) $\int_{0}^{\infty} \gamma\left(e^{e^{x}}\right) d x<\infty$ or equivalently $\int_{e}^{\infty} \frac{\gamma(y)}{y \log y} d y<\infty$,

then $\alpha^{n} \log \left(1+\mathcal{Z}_{n}\right)$ converges almost surely to a non-degenerate finite random variable $Y$ with $\mathbb{P}(Y=0)$ equal to the extinction probability of $\left\{\mathcal{Z}_{n}\right\}$, whereas $Y$ admits a density on $(0, \infty)$. Therefore, also $\alpha^{n} \log \left(\mathcal{Z}_{n} \vee 1\right)$ converges to $Y$ almost surely.

The conditions of Davies quoted as (i-iii) simplify earlier work by Seneta [34. For example, for $\{g\}$ in (1.10), the above is valid with $\alpha=\tau-2$ and $\gamma(x)=C(\log x)^{-1}$, where $C$ is sufficiently large. We prove in Lemma A.1.1 below that for $F$ as in Assumption 1.1 and $G$ the distribution function of $g$ in (1.8), the conditions (i-iii) are satisfied with $\alpha=\tau-2$ and $\gamma(x)=C(\log x)^{\gamma-1}$. In particular, for (iii), we need that $\gamma<1$. 
Let $Y^{(1)}$ and $Y^{(2)}$ be two independent copies of the limit random variable $Y$. In the course of the proof, we will encounter the random variable $M=\min _{t \in \mathbb{Z}}\left(\kappa^{t} Y^{(1)}+\kappa^{c-t} Y^{(2)}\right)$, for some $c \in\{0,1\}$, and where $\kappa=(\tau-2)^{-1}$. The proof relies on the fact that, conditionally on $Y^{(1)} Y^{(2)}>0, M$ has a density. The proof of this fact is as follows. The function $\left(y_{1}, y_{2}\right) \mapsto \min _{t \in \mathbb{Z}}\left(\kappa^{t} y_{1}+\kappa^{c-t} y_{2}\right)$ is discontinuous precisely in the points $\left(y_{1}, y_{2}\right)$ satisfying $\sqrt{y_{2} / y_{1}}=\kappa^{n+\frac{1}{2} c}, n \in \mathbb{Z}$, and, conditionally on $Y^{(1)} Y^{(2)}>0$, the random variables $Y^{(1)}$ and $Y^{(2)}$ are independent continuous random variables. Therefore, conditionally on $Y^{(1)} Y^{(2)}>0$, the random variable $M=\min _{t \in \mathbb{Z}}\left(\kappa^{t} Y^{(1)}+\kappa^{c-t} Y^{(2)}\right)$ has a density.

\section{The growth of the shortest path graph}

In this section, we describe the growth of the shortest path graph (SPG). As a result, we will see that this growth is closely related to a $\mathrm{BP}\left\{\hat{Z}_{k}^{(1, N)}\right\}$ with the random offspring distribution $\left\{g_{j}^{(N)}\right\}$ given by

$$
\begin{aligned}
g_{j}^{(N)} & =\sum_{i=1}^{N} \mathbf{1}_{\left\{D_{i}=j+1\right\}} \mathbb{P}\left(\text { a stub from node } i \text { is sampled } \mid D_{1}, \ldots, D_{N}\right) \\
& =\sum_{i=1}^{N} \mathbf{1}_{\left\{D_{i}=j+1\right\}} \frac{D_{i}}{L_{N}}=\frac{j+1}{L_{N}} \sum_{i=1}^{N} \mathbf{1}_{\left\{D_{i}=j+1\right\}},
\end{aligned}
$$

where, for an event $A, \mathbf{1}_{A}$ denotes the indicator function of the event $A$. By the strong law of large numbers for $N \rightarrow \infty$, almost surely,

$$
\frac{L_{N}}{N} \rightarrow \mathbb{E}[D], \quad \text { and } \quad \frac{1}{N} \sum_{i=1}^{N} \mathbf{1}_{\left\{D_{i}=j+1\right\}} \rightarrow \mathbb{P}(D=j+1),
$$

so that a.s.,

$$
g_{j}^{(N)} \rightarrow(j+1) \mathbb{P}(D=j+1) / \mathbb{E}[D]=g_{j}, \quad N \rightarrow \infty .
$$

Therefore, the BP $\left\{\hat{Z}_{k}^{(1, N)}\right\}$, with offspring distribution $\left\{g_{j}^{(N)}\right\}$, is expected to be close to a BP with offspring distribution $\left\{g_{j}\right\}$ given in (1.8). Consequently, in Section 3.1, we state bounds on the coupling of the BP $\left\{\hat{Z}_{k}^{(1, N)}\right\}$ to a BP $\left\{\mathcal{Z}_{k}^{(1)}\right\}$ with offspring distribution $\left\{g_{j}\right\}$. This allows us to prove Theorems 1.2 and 1.5 in Section 4 .

The shortest path graph (SPG) from node 1 is the power law random graph as observed from node 1 , and consists of the shortest paths between node 1 and all other nodes $\{2, \ldots, N\}$. As will be shown below, the SPG is not necessarily a tree because cycles may occur. Recall that two stubs together form an edge. We define $Z_{1}^{(1, N)}=D_{1}$ and, for $k \geq 2$, we denote by $Z_{k}^{(1, N)}$ the number of stubs attached to nodes at distance $k-1$ from node 1 , but are not part of an edge connected to a node at distance $k-2$. We refer to such stubs as 'free stubs'. Thus, $Z_{k}^{(1, N)}$ is the number of outgoing stubs from nodes at distance $k-1$. By $\mathrm{SPG}_{k-1}$ we denote the SPG up to level $k-1$, i.e., up to the moment we have $Z_{k}^{(1, N)}$ free stubs attached to nodes on distance $k-1$, and no stubs to nodes on distance $k$. Since we compare $Z_{k}^{(1, N)}$ to the $k^{\text {th }}$ generation of the $\mathrm{BP} \hat{Z}_{k}^{(1)}$, we call $Z_{k}^{(1, N)}$ the stubs of level $k$.

The first stages of a realization of the generation of the SPG, with $N=9$ and $L_{N}=24$, are drawn in Figure 3. The first line shows the $N$ different nodes with their attached stubs. Initially, all stubs have label 1 . The growth process starts by choosing the first stub of node 1 whose stubs are labeled by 2 as illustrated in the second line, while all the other stubs maintain the label 1 . Next, we uniformly choose a stub with label 1 or 2 . In the example in line 3 , this is the second stub from node 3 , whose stubs are labeled by 2 and the second stub by label 3 . The left hand side column visualizes the growth of the SPG by the attachment of stub 2 of node 3 to the first stub 


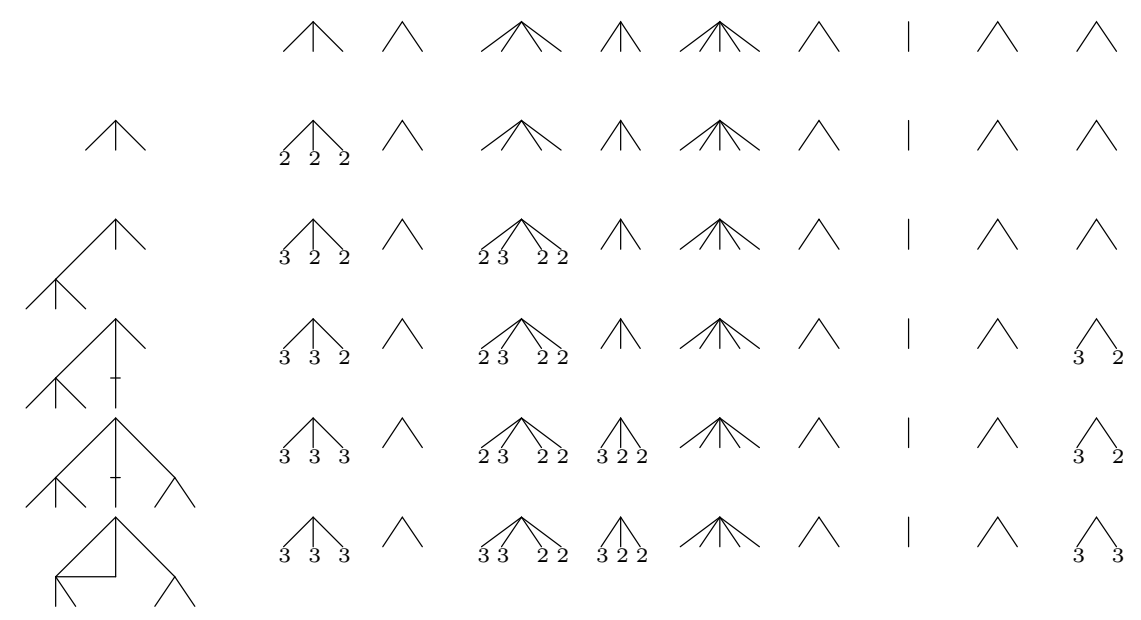

Figure 3: Schematic drawing of the growth of the SPG from the node 1 with $N=9$ and the updating of the labels.

of node 1. Once an edge is established the pairing stubs are labeled 3. In the next step, again a stub is chosen uniformly out of those with label 1 or 2 . In the example in line 4 , it is the first stub of the last node that will be attached to the second stub of node 1 , the next in sequence to be paired. The last line exhibits the result of creating a cycle when the second stub of the last node is chosen to be attached to the first stub of node 3, which is the next stub in the sequence to be paired. This process is continued until there are no more stubs with labels 1 or 2 . In this example, we have $Z_{1}^{(1,9)}=3$ and $Z_{2}^{(1,9)}=6$.

We now describe the meaning of the labels. Initially, all stubs are labeled 1. At each stage of the growth of the SPG, we draw uniformly at random from all stubs with labels 1 and 2 . After each draw we will update the realization of the SPG, and classify the stubs according to three categories, which will be labeled 1,2 and 3. These labels will be updated as the growth of the SPG proceeds. At any stage of the generation of the SPG, the labels have the following meaning:

1. Stubs with label 1 are stubs belonging to a node that is not yet attached to the SPG.

2. Stubs with label 2 are attached to the shortest path graph (because the corresponding node has been chosen), but not yet paired with another stub. These are called 'free stubs'.

3. Stubs with label 3 in the SPG are paired with another stub to form an edge in the SPG.

The growth process as depicted in Figure 3 starts by labelling all stubs by 1 . Then, because we construct the SPG starting from node 1 we relabel the $D_{1}$ stubs of node 1 with the label 2 . We note that $Z_{1}^{(1, N)}$ is equal to the number of stubs connected to node 1 , and thus $Z_{1}^{(1, N)}=D_{1}$. We next identify $Z_{j}^{(1, N)}$ for $j>1 . Z_{j}^{(1, N)}$ is obtained by sequentially growing the SPG from the free stubs in generation $Z_{j-1}^{(1, N)}$. When all free stubs in generation $j-1$ have chosen their connecting stub, $Z_{j}^{(1, N)}$ is equal to the number of stubs labeled 2 (i.e., free stubs) attached to the SPG. Note that not necessarily each stub of $Z_{j-1}^{(1, N)}$ contributes to stubs of $Z_{j}^{(1, N)}$, because a cycle may 'swallow' two free stubs. This is the case when a stub with label 2 is chosen. 
When a stub is chosen, we update the labels as follows:

1. If the chosen stub has label 1 , in the SPG we connect the present stub to the chosen stub to form an edge and attach the remaining stubs of the chosen node as children. We update the labels as follows. The present and chosen stub melt together to form an edge and both are assigned label 3. All 'brother' stubs (except for the chosen stub) belonging to the same node of the chosen stub receive label 2 .

2. In this case we choose a stub with label 2, which is already connected to the SPG. For the SPG, a self-loop is created if the chosen stub and present stub are 'brother' stubs which belong to the same node. If they are not 'brother' stubs, then a cycle is formed. Neither a self-loop nor a cycle changes the distances to the root in the SPG.

The updating of the labels solely consists of changing the label of the present and the chosen stub from 2 to 3 .

The above process stops in the $j^{\text {th }}$ generation when there are no more free stubs in generation $j-1$ for the SPG.

We continue the above process of drawing stubs until there are no more stubs having label 1 or 2 , so that all stubs have label 3. Then, the SPG from node 1 is finalized, and we have generated the shortest path graph as seen from node 1 . We have thus obtained the structure of the shortest path graph, and know how many nodes there are at a given distance from node 1 .

The above construction will be performed identically from node 2 , and we denote the number of free stubs in the SPG of node 2 in generation $k$ by $Z_{k}^{(2, N)}$. This construction is close to being independent. In particular, it is possible to couple the two SPG growth processes with two independent BP's. This is described in detail in [21, Section 3]. We make essential use of the coupling between the SPG's and the BP's, in particular, of [21, Proposition A.3.1] in the appendix. This completes the construction of the SPG's from both node 1 and 2.

\subsection{Bounds on the coupling}

We now investigate the growth of the SPG, and its relationship to the BP with law $g$. In its statement, we write, for $i=1,2$,

$$
Y_{n}^{(i, N)}=(\tau-2)^{n} \log \left(Z_{n}^{(i, N)} \vee 1\right) \quad \text { and } \quad Y_{n}^{(i)}=(\tau-2)^{n} \log \left(\mathcal{Z}_{n}^{(i)} \vee 1\right),
$$

where $\left\{\mathcal{Z}_{j}^{(1)}\right\}_{j \geq 1}$ and $\left\{\mathcal{Z}_{j}^{(2)}\right\}_{j \geq 1}$ are two independent delayed BP's with offspring distribution $\{g\}$ and where $\mathcal{Z}_{1}^{(i)}$ has law $\{f\}$. Then the following proposition shows that the first levels of the SPG are close to those of the BP's:

Proposition 3.1 (Coupling at fixed time) For every $m$ fixed, and for $i=1,2$, there exist independent delayed $B P^{\prime}$ 's $\mathcal{Z}^{(1)}, \mathcal{Z}^{(2)}$, such that

$$
\lim _{N \rightarrow \infty} \mathbb{P}\left(Y_{m}^{(i, N)}=Y_{m}^{(i)}\right)=1 .
$$

In words, Proposition 3.1 states that at any fixed time, the SPG's from 1 and 2 can be coupled to two independent BP's with offspring $g$, in such a way that the probability that the SPG differs from the BP vanishes when $N \rightarrow \infty$.

In the statement of the next proposition, we write, for $i=1,2$,

$$
\begin{aligned}
\mathcal{T}_{m}^{(i, N)}=\mathcal{T}_{m}^{(i, N)}(\varepsilon) & =\left\{k>m:\left(Z_{m}^{(i, N)}\right)^{\kappa^{k-m}} \leq N^{\frac{1-\varepsilon^{2}}{\tau-1}}\right\} \\
& =\left\{k>m: \kappa^{k} Y_{m}^{(i, N)} \leq \frac{1-\varepsilon^{2}}{\tau-1} \log N\right\},
\end{aligned}
$$


where we recall that $\kappa=(\tau-2)^{-1}$.

We will see that $Z_{k}^{(i, N)}$ grows super-exponentially with $k$ as long as $k \in \mathcal{T}_{m}^{(i, N)}$. More precisely, $\left(Z_{m}^{(i, N)}\right)^{\kappa^{k-m}}$ is close to $Z_{k}^{(i, N)}$, and thus, $\mathcal{T}_{m}^{(i, N)}$ can be thought of as the generations for which the generation size is bounded by $N^{\frac{1-\varepsilon^{2}}{\tau-1}}$. The second main result of the coupling is the following proposition:

Proposition 3.2 (Super-exponential growth with base $Y_{m}^{(i, N)}$ for large times) If $F$ satisfies Assumption 1.1, then for $i=1,2$,

$$
\begin{aligned}
\text { (a) } \quad & \mathbb{P}\left(\varepsilon \leq Y_{m}^{(i, N)} \leq \varepsilon^{-1}, \max _{k \in \mathcal{T}_{m}^{(i, N)}(\varepsilon)}\left|Y_{k}^{(i, N)}-Y_{m}^{(i, N)}\right|>\varepsilon^{3}\right)=o_{N, m \varepsilon}(1), \\
\text { (b) } \quad & \mathbb{P}\left(\varepsilon \leq Y_{m}^{(i, N)} \leq \varepsilon^{-1}, \exists k \in \mathcal{T}_{m}^{(i, N)}(\varepsilon): Z_{k-1}^{(i, N)}>Z_{k}^{(i, N)}\right)=o_{N, m \varepsilon}(1), \\
& \mathbb{P}\left(\varepsilon \leq Y_{m}^{(i, N)} \leq \varepsilon^{-1}, \exists k \in \mathcal{T}_{m}^{(i, N)}(\varepsilon): Z_{k}^{(i, N)}>N^{\frac{1-\varepsilon^{4}}{\tau-1}}\right)=o_{N, m \varepsilon}(1),
\end{aligned}
$$

where $o_{N, m \varepsilon}(1)$ denotes a quantity $\gamma_{N, m, \varepsilon}$ that converges to zero when first $N \rightarrow \infty$, then $m \rightarrow \infty$ and finally $\varepsilon \downarrow 0$.

Proposition 3.2 (a), i.e., (3.6), is the main coupling result used in this paper, and says that as long as $k \in \mathcal{T}_{m}^{(i, N)}(\varepsilon)$, we have that $Y_{k}^{(i, N)}$ is close to $Y_{m}^{(i, N)}$, which, in turn, by Proposition 3.1 is close to $Y_{m}^{(i)}$. This establishes the coupling between the SPG and the BP. Part (b) is a technical result used in the proof. Equation (3.7) is a convenient result, as it shows that, with high probability, $k \mapsto Z_{k}^{(i, N)}$ is monotone increasing. Equation (3.8) shows that with high probability $Z_{k}^{(i, N)} \leq N^{\frac{1-\varepsilon^{4}}{\tau-1}}$ for all $k \in \mathcal{T}_{m}^{(i, N)}(\varepsilon)$, which allows us to bound the number of free stubs in generation sizes that are in $\mathcal{T}_{m}^{(i, N)}(\varepsilon)$.

We complete this section with a final coupling result, which shows that for the first $k$ which is not in $\mathcal{T}_{m}^{(i, N)}(\varepsilon)$, the SPG has many free stubs:

Proposition 3.3 (Lower bound on $Z_{k+1}^{(i, N)}$ for $k+1 \notin \mathcal{T}_{m}^{(i, N)}(\varepsilon)$ ) Let F satisfy Assumption [1.1. Then,

$$
\mathbb{P}\left(k \in \mathcal{T}_{m}^{(i, N)}(\varepsilon), k+1 \notin \mathcal{T}_{m}^{(i, N)}(\varepsilon), \varepsilon \leq Y_{m}^{(i, N)} \leq \varepsilon^{-1}, Z_{k+1}^{(i, N)} \leq N^{\frac{1-\varepsilon}{\tau-1}}\right)=o_{N, m, \varepsilon}(1) .
$$

Propositions 3.13 .2 and 3.3 will be proved in the appendix. We now prove the main results in Theorems 1.2 and 1.5 subject to Propositions 3.1, 3.2 and 3.3 in Section 4

\section{Proof of Theorems 1.2 and 1.5}

In this section we prove Theorem 1.2 and identify the limit in Theorem 1.5 using the coupling theory of the previous section. For $i=1,2$, we recall that $Z_{j}^{(i, N)}$ is the number of free stubs connected to nodes on distance $j-1$ from root $i$. As we show in this section, the hopcount $H_{N}$ is closely related to the SPG's $\left\{Z_{j}^{(i, N)}\right\}_{j \geq 0}, i=1,2$.

\subsection{Outline of the proof}

We start by describing the outline of the proof. The proof is divided into several key steps proved in 5 subsections.

In the first key step of the proof, in Section 4.2, we split the probability $\mathbb{P}\left(H_{N}>k\right)$ into separate parts depending on the values of $Y_{m}^{(i, N)}=(\tau-2)^{m} \log \left(Z_{m}^{(i, N)} \vee 1\right)$. We prove that

$$
\mathbb{P}\left(H_{N}>k, Y_{m}^{(1, N)} Y_{m}^{(2, N)}=0\right)=1-q_{m}^{2}+o(1), \quad N \rightarrow \infty,
$$


where $1-q_{m}$ is the probability that the delayed $\mathrm{BP}\left\{\mathcal{Z}_{j}^{(1)}\right\}_{j \geq 1}$ dies at or before the $m^{\text {th }}$ generation. When $m$ becomes large, then $q_{m} \rightarrow q$, where $q$ equals the survival probability of the $\operatorname{BP}\left\{\mathcal{Z}_{j}^{(1)}\right\}_{j \geq 1}$. This leaves us to determine the contribution to $\mathbb{P}\left(H_{N}>k\right)$ for the cases where $Y_{m}^{(1, N)} Y_{m}^{(2, N)}>0$. We further show that for $m$ large enough, and on the event that $Y_{m}^{(i, N)}>0$, whp, $Y_{m}^{(i, N)} \in\left[\varepsilon, \varepsilon^{-1}\right]$, for $i=1,2$, where $\varepsilon>0$ is small. This provides us with a priori bounds on the shortest path graph exploration processes $\left\{Z_{j}^{(i, N)}\right\}$. We denote the event where $Y_{m}^{(i, N)} \in\left[\varepsilon, \varepsilon^{-1}\right]$, for $i=1,2$, by $E_{m, N}(\varepsilon)$.

The second key step in the proof, in Section 4.3, is to obtain an asymptotic formula for $\mathbb{P}\left(\left\{H_{N}>\right.\right.$ $\left.k\} \cap E_{m, N}(\varepsilon)\right)$. Indeed, we prove the existence of $\lambda=\lambda_{N}(k)>0$ such that

$$
\mathbb{P}\left(\left\{H_{N}>k\right\} \cap E_{m, N}(\varepsilon)\right)=\mathbb{E}\left[\mathbf{1}_{E_{m, N}(\varepsilon)} \exp \left\{-\lambda \frac{Z_{k_{1}+1}^{(1, N)} Z_{k-k_{1}}^{(2, N)}}{L_{N}}\right\}\right],
$$

where the right-hand side is valid for any $k_{1}$ with $0 \leq 2 k_{1} \leq k-1$, and where $\lambda=\lambda_{N}(k)$ satisfies $\frac{1}{2} \leq \lambda_{N}(k) \leq 4 k$. It is even allowed that $k_{1}$ is random, as long as it is measurable w.r.t. $\left\{Z_{j}^{(i, N)}\right\}_{j=1}^{m}$. Even though the estimate on $\lambda_{N}$ is not sharp, it turns out that it gives us enough information to complete the proof. The bounds $\frac{1}{2} \leq \lambda_{N}(k) \leq 4 k$ play a crucial role in the remainder of the proof.

In the third key step, in Section 4.4 we show that, for $k=k_{N} \rightarrow \infty$, the main contribution of (4.2) stems from the term

$$
\mathbb{E}\left[\mathbf{1}_{E_{m, N}(\varepsilon)} \exp \left\{-\lambda \min _{k_{1} \in \mathcal{B}_{N}} \frac{Z_{k_{1}+1}^{(1, N)} Z_{k_{N}-k_{1}}^{(2, N)}}{L_{N}}\right\}\right],
$$

with $\mathcal{B}_{N}=\mathcal{B}_{N}\left(\varepsilon, k_{N}\right)$ defined in (4.50) and is such that $k_{1} \in \mathcal{B}_{N}\left(\varepsilon, k_{N}\right)$ precisely when $k_{1}+1 \in$ $\mathcal{T}_{m}^{(1, N)}(\varepsilon)$ and $k_{N}-k_{1} \in \mathcal{T}_{m}^{(2, N)}(\varepsilon)$. Thus, by Proposition 3.2, it implies that whp

$$
Z_{k_{1}+1}^{(1, N)} \leq N^{\frac{1-\varepsilon^{4}}{\tau-1}} \quad \text { and } \quad Z_{k_{N}-k_{1}}^{(2, N)} \leq N^{\frac{1-\varepsilon^{4}}{\tau-1}} .
$$

In turn, these bounds allow us to use Proposition 3.2 (a).

In the fourth key step, in Section 4.5 we proceed by choosing

$$
k_{N}=2\left\lfloor\frac{\log \log N}{|\log (\tau-2)|}\right\rfloor+l,
$$

and we show that with probability converging to 1 as $\varepsilon \downarrow 0$, the results of the coupling in Proposition 3.2 apply, which implies that $Y_{k_{1}+1}^{(1, N)} \approx Y_{m}^{(1, N)}$ and $Y_{k_{N}-k_{1}}^{(2, N)} \approx Y_{m}^{(2, N)}$.

In the final key step, in Section 4.6. the minimum occurring in (4.3), with the approximations $Y_{k_{1}+1}^{(1, N)} \approx Y_{m}^{(1, N)}$ and $Y_{k_{N}-k_{1}}^{(2, N)} \approx Y_{m}^{(2, N)}$, is analyzed. The main idea in this analysis is as follows. With the above approximations, the expression in (4.3) can be rewritten as

$$
\mathbb{E}\left[\mathbf{1}_{E_{m, N}(\varepsilon)} \exp \left\{-\lambda \exp \left[\min _{k_{1} \in \mathcal{B}_{N}\left(\varepsilon, k_{N}\right)}\left(\kappa^{k_{1}+1} Y_{m}^{(1, N)}+\kappa^{k_{N}-k_{1}} Y_{m}^{(2, N)}\right)-\log L_{N}\right]\right\}\right]+o_{N, m, \varepsilon}(1)
$$

where $\kappa=(\tau-2)^{-1}>1$. The minimum appearing in the exponent of (4.5) is then rewritten (see (4.72) and (4.74) ) as

$$
\kappa^{\left\lceil k_{N} / 2\right\rceil}\left\{\min _{t \in \mathbb{Z}}\left(\kappa^{t} Y_{m}^{(1, N)}+\kappa^{c_{l}-t} Y_{m}^{(2, N)}\right)-\kappa^{-\left\lceil k_{N} / 2\right\rceil} \log L_{N}\right\} .
$$

Since $\kappa^{\left\lceil k_{N} / 2\right\rceil} \rightarrow \infty$, the latter expression only contributes to 4.5] when

$$
\min _{t \in \mathbb{Z}}\left(\kappa^{t} Y_{m}^{(1, N)}+\kappa^{c_{l}-t} Y_{m}^{(2, N)}\right)-\kappa^{-\left\lceil k_{N} / 2\right\rceil} \log L_{N} \leq 0 .
$$

Here it will become apparent that the bounds on $\lambda_{N}(k)$ are sufficient. The expectation of the indicator of this event leads to the peculiar limit

$$
\mathbb{P}\left(\min _{t \in \mathbb{Z}}\left(\kappa^{t} Y^{(1)}+\kappa^{c_{l}-t} Y^{(2)}\right) \leq \kappa^{a_{N}-\lceil l / 2\rceil}, Y^{(1)} Y^{(2)}>0\right),
$$

with $a_{N}$ and $c_{l}$ as defined in Theorem 1.2. We complete the proof by showing that conditioning on the event that 1 and 2 are connected is asymptotically equivalent to conditioning on $Y^{(1)} Y^{(2)}>0$. 
Remark 4.1 In the course of the proof, we will see that it is not necessary that the degrees of the nodes are i.i.d. In fact, in the proof below, we need that Propositions 3.1 3.3 are valid, as well as that $L_{N}$ is concentrated around its mean $\mu N$. In Remark A.1.5 in the appendix, we will investigate what is needed in the proof of Propositions 3.1-3.3. In particular, the proof applies also to some instances of the configuration model where the number of nodes with degree $k$ is fixed, when we investigate the distance between two uniformly chosen nodes.

We now go through the details of the proof.

\subsection{A priory bounds on $Y_{m}^{(i, N)}$}

We wish to compute the probability $\mathbb{P}\left(H_{N}>k\right)$. To do so, we split $\mathbb{P}\left(H_{N}>k\right)$ as

$$
\mathbb{P}\left(H_{N}>k\right)=\mathbb{P}\left(H_{N}>k, Y_{m}^{(1, N)} Y_{m}^{(2, N)}=0\right)+\mathbb{P}\left(H_{N}>k, Y_{m}^{(1, N)} Y_{m}^{(2, N)}>0\right),
$$

where we take $m$ to be sufficiently large. We will now prove two lemmas, and use these to compute the first term in the right-hand side of (4.6).

\section{Lemma 4.2}

$$
\lim _{N \rightarrow \infty} \mathbb{P}\left(Y_{m}^{(1, N)} Y_{m}^{(2, N)}=0\right)=1-q_{m}^{2}
$$

where

$$
q_{m}=\mathbb{P}\left(Y_{m}^{(1)}>0\right)
$$

Proof. By Proposition 3.1 for $N \rightarrow \infty$, and because $Y_{m}^{(1)}$ and $Y_{m}^{(2)}$ are independent,

$$
\begin{aligned}
\mathbb{P}\left(Y_{m}^{(1, N)} Y_{m}^{(2, N)}=0\right) & =\mathbb{P}\left(Y_{m}^{(1)} Y_{m}^{(2)}=0\right)+o(1)=1-\mathbb{P}\left(Y_{m}^{(1)} Y_{m}^{(2)}>0\right)+o(1) \\
& =1-\mathbb{P}\left(Y_{m}^{(1)}>0\right) \mathbb{P}\left(Y_{m}^{(2)}>0\right)+o(1)=1-q_{m}^{2}+o(1) .
\end{aligned}
$$

The following lemma shows that the probability that $H_{N} \leq m$ converges to zero for any fixed $m:$

Lemma 4.3 For any $m$ fixed,

$$
\lim _{N \rightarrow \infty} \mathbb{P}\left(H_{N} \leq m\right)=0 .
$$

Proof. As observed above Theorem 1.2 by exchangeability of the nodes $\{1,2, \ldots, N\}$,

$$
\mathbb{P}\left(H_{N} \leq m\right)=\mathbb{P}\left(\widetilde{H}_{N} \leq m\right),
$$

where $\widetilde{H}_{N}$ is the hopcount between node 1 and a uniformly chosen node unequal to 1 . We split, for any $0<\delta<1$,

$$
\mathbb{P}\left(\widetilde{H}_{N} \leq m\right)=\mathbb{P}\left(\widetilde{H}_{N} \leq m, \sum_{j \leq m} Z_{j}^{(1, N)} \leq N^{\delta}\right)+\mathbb{P}\left(\widetilde{H}_{N} \leq m, \sum_{j \leq m} Z_{j}^{(1, N)}>N^{\delta}\right) .
$$

The number of nodes at distance at most $m$ from node 1 is bounded from above by $\sum_{j \leq m} Z_{j}^{(1, N)}$. The event $\left\{\widetilde{H}_{N} \leq m\right\}$ can only occur when the end node, which is uniformly chosen in $\{2, \ldots, N\}$, is in the SPG of node 1, so that

$$
\mathbb{P}\left(\widetilde{H}_{N} \leq m, \sum_{j \leq m} Z_{j}^{(1, N)} \leq N^{\delta}\right) \leq \frac{N^{\delta}}{N-1}=o(1), \quad N \rightarrow \infty .
$$


Therefore, the first term in (4.9) is $o(1)$, as required. We will proceed with the second term in (4.9). By Proposition 3.1 whp, we have that $Y_{j}^{(1, N)}=Y_{j}^{(1)}$ for all $j \leq m$. Therefore, we obtain, because $Y_{j}^{(1, N)}=Y_{j}^{(1)}$ implies $Z_{j}^{(1, N)}=\mathcal{Z}_{j}^{(1)}$,

$$
\mathbb{P}\left(\widetilde{H}_{N} \leq m, \sum_{j \leq m} Z_{j}^{(1, N)}>N^{\delta}\right) \leq \mathbb{P}\left(\sum_{j \leq m} Z_{j}^{(1, N)}>N^{\delta}\right)=\mathbb{P}\left(\sum_{j \leq m} \mathcal{Z}_{j}^{(1)}>N^{\delta}\right)+o(1) .
$$

However, when $m$ is fixed, the random variable $\sum_{j \leq m} \mathcal{Z}_{j}^{(1)}$ is finite with probability 1 , and therefore,

$$
\lim _{N \rightarrow \infty} \mathbb{P}\left(\widetilde{H}_{N} \leq m, \sum_{j \leq m} Z_{j}^{(1, N)}>N^{\delta}\right)=0 .
$$

This completes the proof of Lemma 4.3 .

We now use Lemmas 4.2 and 4.3 to compute the first term in (4.6). We split

$$
\mathbb{P}\left(H_{N}>k, Y_{m}^{(1, N)} Y_{m}^{(2, N)}=0\right)=\mathbb{P}\left(Y_{m}^{(1, N)} Y_{m}^{(2, N)}=0\right)-\mathbb{P}\left(H_{N} \leq k, Y_{m}^{(1, N)} Y_{m}^{(2, N)}=0\right) .
$$

By Lemma 4.2, the first term is equal to $1-q_{m}^{2}+o(1)$. For the second term, we note that when $Y_{m}^{(1, N)}=0$ and $H_{N}<\infty$, then $H_{N} \leq m-1$, so that

$$
\mathbb{P}\left(H_{N} \leq k, Y_{m}^{(1, N)} Y_{m}^{(2, N)}=0\right) \leq \mathbb{P}\left(H_{N} \leq m-1\right) .
$$

Using Lemma 4.3. we conclude that

Corollary 4.4 For every $m$ fixed, and each $k \in \mathbb{N}$,

$$
\lim _{N \rightarrow \infty} \mathbb{P}\left(H_{N}>k, Y_{m}^{(1, N)} Y_{m}^{(2, N)}=0\right)=1-q_{m}^{2} .
$$

By Corollary 4.4 and (4.6), we are left to compute $\mathbb{P}\left(H_{N}>k, Y_{m}^{(1, N)} Y_{m}^{(2, N)}>0\right)$. We first prove a lemma that shows that if $Y_{m}^{(1, N)}>0$, then whp $Y_{m}^{(1, N)} \in\left[\varepsilon, \varepsilon^{-1}\right]$ :

Lemma 4.5 For $i=1,2$,

$$
\limsup _{\varepsilon \downarrow 0} \limsup _{m \rightarrow \infty} \limsup _{N \rightarrow \infty} \mathbb{P}\left(0<Y_{m}^{(i, N)}<\varepsilon\right)=\limsup _{\epsilon \downarrow 0} \limsup _{m \rightarrow \infty} \limsup _{N \rightarrow \infty} \mathbb{P}\left(Y_{m}^{(i, N)}>\varepsilon^{-1}\right)=0 .
$$

Proof. Fix $m$, when $N \rightarrow \infty$ it follows from Proposition 3.1 that $Y_{m}^{(i, N)}=Y_{m}^{(i)}$, whp. Thus, we obtain that

$$
\limsup _{\varepsilon \downarrow 0} \limsup _{m \rightarrow \infty} \limsup _{N \rightarrow \infty} \mathbb{P}\left(0<Y_{m}^{(i, N)}<\varepsilon\right)=\limsup _{\epsilon \downarrow 0} \limsup _{m \rightarrow \infty} \mathbb{P}\left(0<Y_{m}^{(i)}<\varepsilon\right),
$$

and similarly for the second probability. The remainder of the proof of the lemma follows because $Y_{m}^{(i)} \stackrel{d}{\rightarrow} Y^{(i)}$ as $m \rightarrow \infty$ and is hence a tight sequence.

Write

$$
\begin{aligned}
E_{m, N} & =E_{m, N}(\varepsilon)=\left\{Y_{m}^{(i, N)} \in\left[\varepsilon, \varepsilon^{-1}\right], i=1,2\right\}, \\
F_{m, N} & =F_{m, N}(\varepsilon)=\left\{\max _{k \in \mathcal{T}_{m}^{(N)}(\varepsilon)}\left|Y_{k}^{(i, N)}-Y_{m}^{(i, N)}\right| \leq \varepsilon^{3}, i=1,2\right\} .
\end{aligned}
$$

As a consequence of Lemma 4.5. we obtain that

$$
\mathbb{P}\left(E_{m, N}^{c} \cap\left\{Y_{m}^{(1, N)} Y_{m}^{(2, N)}>0\right\}\right)=o_{N, m, \varepsilon}(1) .
$$

In the sequel, we compute

$$
\mathbb{P}\left(\left\{H_{N}>k\right\} \cap E_{m, N}\right),
$$

and often we make use of the fact that by Proposition 3.2

$$
\mathbb{P}\left(E_{m, N} \cap F_{m, N}^{c}\right)=o_{N, m, \varepsilon}(1) .
$$




\subsection{Asymptotics of $\mathbb{P}\left(\left\{H_{N}>k\right\} \cap E_{m, N}\right)$}

We next give a representation of $\mathbb{P}\left(\left\{H_{N}>k\right\} \cap E_{m, N}\right)$. In order to do so, we write $\mathbb{Q}_{Z}^{(i, j)}$, where $i, j \geq 0$, for the conditional probability given $\left\{Z_{s}^{(1, N)}\right\}_{s=1}^{i}$ and $\left\{Z_{s}^{(2, N)}\right\}_{s=1}^{j}$ (where, for $j=0$, we condition only on $\left\{Z_{s}^{(1, N)}\right\}_{s=1}^{i}$ ), and $\mathbb{E}_{Z}^{(i, j)}$ for its conditional expectation. Furthermore, we say that a random variable $k_{1}$ is $Z_{m}$-measurable if $k_{1}$ is measurable with respect to the $\sigma$-algebra generated by $\left\{Z_{s}^{(1, N)}\right\}_{s=1}^{m}$ and $\left\{Z_{s}^{(2, N)}\right\}_{s=1}^{m}$. The main rewrite is now in the following lemma:

Lemma 4.6 For $k \geq 2 m-1$,

$$
\mathbb{P}\left(\left\{H_{N}>k\right\} \cap E_{m, N}\right)=\mathbb{E}\left[\mathbf{1}_{E_{m, N}} \mathbb{Q}_{Z}^{(m, m)}\left(H_{N}>2 m-1\right) P_{m}\left(k, k_{1}\right)\right],
$$

where, for any $Z_{m}$-measurable $k_{1}$, with $m \leq k_{1} \leq(k-1) / 2$,

$$
\begin{aligned}
P_{m}\left(k, k_{1}\right)= & \prod_{i=2 m}^{2 k_{1}} \mathbb{Q}_{Z}^{(\lfloor i / 2\rfloor+1,\lceil i / 2\rceil)}\left(H_{N}>i \mid H_{N}>i-1\right) \\
& \times \prod_{i=1}^{k-2 k_{1}} \mathbb{Q}_{Z}^{\left(k_{1}+1, k_{1}+i\right)}\left(H_{N}>2 k_{1}+i \mid H_{N}>2 k_{1}+i-1\right) .
\end{aligned}
$$

Proof. We start by conditioning on $\left\{Z_{s}^{(1, N)}\right\}_{s=1}^{m}$ and $\left\{Z_{s}^{(2, N)}\right\}_{s=1}^{m}$, and note that $E_{m, N}$ is measurable w.r.t. $\left\{Z_{s}^{(1, N)}\right\}_{s=1}^{m}$ and $\left\{Z_{s}^{(2, N)}\right\}_{s=1}^{m}$, so that we obtain, for $k \geq 2 m-1$,

$$
\begin{aligned}
\mathbb{P}\left(\left\{H_{N}>k\right\} \cap E_{m, N}\right) & =\mathbb{E}\left[\mathbf{1}_{E_{m, N}} \mathbb{Q}_{Z}^{(m, m)}\left(H_{N}>k\right)\right] \\
& =\mathbb{E}\left[\mathbf{1}_{E_{m, N}} \mathbb{Q}_{Z}^{(m, m)}\left(H_{N}>2 m-1\right) \mathbb{Q}_{Z}^{(m, m)}\left(H_{N}>k \mid H_{N}>2 m-1\right)\right] .
\end{aligned}
$$

Moreover, for $i, j$ such that $i+j \leq k$,

$$
\begin{aligned}
& \mathbb{Q}_{Z}^{(i, j)}\left(H_{N}>k \mid H_{N}>i+j-1\right) \\
& =\mathbb{E}_{Z}^{(i, j)}\left[\mathbb{Q}_{Z}^{(i, j+1)}\left(H_{N}>k \mid H_{N}>i+j-1\right)\right] \\
& =\mathbb{E}_{Z}^{(i, j)}\left[\mathbb{Q}_{Z}^{(i, j+1)}\left(H_{N}>i+j \mid H_{N}>i+j-1\right) \mathbb{Q}_{Z}^{(i, j+1)}\left(H_{N}>k \mid H_{N}>i+j\right)\right],
\end{aligned}
$$

and, similarly,

$$
\begin{aligned}
& \mathbb{Q}_{Z}^{(i, j)}\left(H_{N}>k \mid H_{N}>i+j-1\right) \\
& =\mathbb{E}_{Z}^{(i, j)}\left[\mathbb{Q}_{Z}^{(i+1, j)}\left(H_{N}>i+j \mid H_{N}>i+j-1\right) \mathbb{Q}_{Z}^{(i+1, j)}\left(H_{N}>k \mid H_{N}>i+j\right)\right] .
\end{aligned}
$$

When we apply the above formulas, we can choose to increase $i$ or $j$ by one depending on $\left\{Z_{s}^{(1, N)}\right\}_{s=1}^{i}$ and $\left\{Z_{s}^{(2, N)}\right\}_{s=1}^{j}$. We iterate the above recursions until $i+j=k-1$. In particular, we obtain, for $k>2 m-1$,

$$
\begin{aligned}
\mathbb{Q}_{Z}^{(m, m)}\left(H_{N}>k \mid H_{N}>2 m-1\right)=\mathbb{E}_{Z}^{(m, m)} & {\left[\mathbb{Q}_{Z}^{(m+1, m)}\left(H_{N}>2 m \mid H_{N}>2 m-1\right)\right.} \\
& \left.\times \mathbb{Q}_{Z}^{(m+1, m)}\left(H_{N}>k \mid H_{N}>2 m\right)\right],
\end{aligned}
$$

so that, using that $E_{m, N}$ is $\mathbb{Q}_{Z}^{(m, m)}$-measurable and that $\mathbb{E}\left[\mathbb{E}_{Z}^{(m, m)}[X]\right]=\mathbb{E}[X]$ for any random variable $X$,

$$
\begin{aligned}
& \mathbb{P}\left(\left\{H_{N}>k\right\} \cap E_{m, N}\right) \\
& =\mathbb{E}\left[\mathbf{1}_{E_{m, N}} \mathbb{Q}_{Z}^{(m, m)}\left(H_{N}>2 m-1\right) \mathbb{Q}_{Z}^{(m+1, m)}\left(H_{N}>2 m \mid H_{N}>2 m-1\right) \mathbb{Q}_{Z}^{(m+1, m)}\left(H_{N}>k \mid H_{N}>2 m\right)\right] .
\end{aligned}
$$


We now compute the conditional probability by increasing $i$ or $j$ as follows. For $i+j \leq 2 k_{1}$, we will increase $i$ and $j$ in turn by 1 , and for $i+j>2 k_{1}$, we will only increase the second component $j$. This leads to

$$
\begin{aligned}
\mathbb{Q}_{Z}^{(m, m)}\left(H_{N}>k \mid H_{N}>2 m-1\right)= & \mathbb{E}_{Z}^{(m, m)}\left[\prod_{i=2 m}^{2 k_{1}} \mathbb{Q}_{Z}^{(\lfloor i / 2\rfloor+1,\lceil i / 2\rceil)}\left(H_{N}>i \mid H_{N}>i-1\right)\right. \\
& \left.\times \prod_{j=1}^{k-2 k_{1}} \mathbb{Q}_{Z}^{\left(k_{1}+1, k_{1}+j\right)}\left(H_{N}>2 k_{1}+j \mid H_{N}>2 k_{1}+j-1\right)\right] \\
= & \mathbb{E}_{Z}^{(m, m)}\left[P_{m}\left(k, k_{1}\right)\right] .
\end{aligned}
$$

Here, we use that we can move the expectations $\mathbb{E}_{z}^{(i, j)}$ outside, as in (4.25), so that these do not appear in the final formula. Therefore, from (4.21) and (4.26),

$$
\begin{aligned}
\mathbb{P}\left(\left\{H_{N}>k\right\} \cap E_{m, N}\right) & =\mathbb{E}\left[\mathbf{1}_{E_{m, N}} \mathbb{Q}_{Z}^{(m, m)}\left(H_{N}>2 m-1\right) \mathbb{E}_{Z}^{(m, m)}\left[P_{m}\left(k, k_{1}\right)\right]\right] \\
& =\mathbb{E}\left[\mathbb{E}_{Z}^{(m, m)}\left[\mathbf{1}_{E_{m, N}} \mathbb{Q}_{Z}^{(m, m)}\left(H_{N}>2 m-1\right) P_{m}\left(k, k_{1}\right)\right]\right] \\
& =\mathbb{E}\left[\mathbf{1}_{E_{m, N}} \mathbb{Q}_{Z}^{(m, m)}\left(H_{N}>2 m-1\right) P_{m}\left(k, k_{1}\right)\right] .
\end{aligned}
$$

This proves (4.20).

We note that we can omit the term $\mathbb{Q}_{Z}^{(m, m)}\left(H_{N}>2 m-1\right)$ in (4.19) by introducing a small error term. Indeed, we can write

$$
\mathbb{Q}_{Z}^{(m, m)}\left(H_{N}>2 m-1\right)=1-\mathbb{Q}_{Z}^{(m, m)}\left(H_{N} \leq 2 m-1\right) .
$$

For the contribution to (4.19) due to the second term in (4.28), we bound $\mathbf{1}_{E_{m, N}} P_{m}\left(k, k_{1}\right) \leq 1$. Therefore, the contribution to (4.19) due to the second term in (4.28) is bounded by

$$
\mathbb{E}\left[\mathbb{Q}_{Z}^{(m, m)}\left(H_{N} \leq 2 m-1\right)\right]=\mathbb{P}\left(H_{N} \leq 2 m-1\right)=o_{N}(1),
$$

by Lemma 4.3 .

We conclude that by (4.29), (4.18) and (4.19),

$$
\mathbb{P}\left(\left\{H_{N}>k\right\} \cap E_{m, N}\right)=\mathbb{E}\left[\mathbf{1}_{E_{m, N} \cap F_{m, N}} P_{m}\left(k, k_{1}\right)\right]+o_{N, m, \varepsilon}(1),
$$

where we recall (4.20) for the conditional probability $P_{m}\left(k, k_{1}\right)$ appearing in (4.30).

We continue with (4.30) by investigating the conditional probabilities in $P_{m}\left(k, k_{1}\right)$ defined in (4.20). We have the following bounds for $\mathbb{Q}_{Z}^{(i+1, j)}\left(H_{N}>i+j \mid H_{N}>i+j-1\right)$ :

Lemma 4.7 For all integers $i, j \geq 0$,

$$
\exp \left\{-\frac{4 Z_{i+1}^{(1, N)} Z_{j}^{(2, N)}}{L_{N}}\right\} \leq \mathbb{Q}_{Z}^{(i+1, j)}\left(H_{N}>i+j \mid H_{N}>i+j-1\right) \leq \exp \left\{-\frac{Z_{i+1}^{(1, N)} Z_{j}^{(2, N)}}{2 L_{N}}\right\} .
$$

The upper bound is always valid, the lower bound is valid whenever

$$
\sum_{s=1}^{i+1} Z_{s}^{(1, N)}+\sum_{s=1}^{j} Z_{s}^{(2, N)} \leq \frac{L_{N}}{4}
$$


Proof. We start with the upper bound. We fix two sets of $n_{1}$ and $n_{2}$ stubs, and will be interested in the probability that none of the $n_{1}$ stubs are connected to the $n_{2}$ stubs. We order the $n_{1}$ stubs in an arbitrary way, and connect the stubs iteratively to other stubs. Note that we must connect at least $\left\lceil n_{1} / 2\right\rceil$ stubs, since any stub that is being connected removes at most 2 stubs from the total of $n_{1}$ stubs. The number $n_{1} / 2$ is reached for $n_{1}$ even precisely when all the $n_{1}$ stubs are connected with each other. Therefore, we obtain that the probability that the $n_{1}$ stubs are not connected to the $n_{2}$ stubs is bounded from above by

$$
\prod_{i=1}^{\left\lceil n_{1} / 2\right\rceil}\left(1-\frac{n_{2}}{L_{N}-2 i+1}\right)
$$

To complete the upper bound, we note that

$$
1-\frac{n_{2}}{L_{N}-2 i+1} \leq 1-\frac{n_{2}}{L_{N}} \leq e^{-\frac{n_{2}}{L_{N}}}
$$

to obtain that the probability that the $n_{1}$ stubs are not connected to the $n_{2}$ stubs is bounded from above by

$$
e^{-\left\lceil n_{1} / 2\right\rceil \frac{n_{2}}{L_{N}}} \leq e^{-\frac{n_{1} n_{2}}{2 L_{N}}}
$$

Applying the above bound to $n_{1}=Z_{i+1}^{(1, N)}$ and $n_{2}=Z_{j}^{(2, N)}$, and noting that the probability that $H_{N}>i+j$ given that $H_{N}>i+j-1$ is bounded from above by the probability that the stubs in $Z_{i+1}^{(1, N)}$ are not connected to the stubs in $Z_{j}^{(2, N)}$ leads to

$$
\mathbb{Q}_{Z}^{(i+1, j)}\left(H_{N}>i+j \mid H_{N}>i+j-1\right) \leq \exp \left\{-\frac{Z_{i+1}^{(1, N)} Z_{j}^{(2, N)}}{2 L_{N}}\right\},
$$

which completes the proof of the upper bound.

We again fix two sets of $n_{1}$ and $n_{2}$ stubs, and are again interested in the probability that none of the $n_{1}$ stubs are connected to the $n_{2}$ stubs. However, now we use these bounds repeatedly, and we assume that in each step there remain to be at least $M$ stubs available. We order the $n_{1}$ stubs in an arbitrary way, and connect the stubs iteratively to other stubs. We obtain a lower bound by further requiring that the $n_{1}$ stubs do not connect to each other. Therefore, the probability that the $n_{1}$ stubs are not connected to the $n_{2}$ stubs is bounded below by

$$
\prod_{i=1}^{n_{1}}\left(1-\frac{n_{2}}{M-2 i+1}\right)
$$

When $M-2 n_{1} \geq \frac{L_{N}}{2}$, we obtain that $1-\frac{n_{2}}{M-2 i+1} \geq 1-\frac{2 n_{2}}{L_{N}}$. Moreover, when $x \leq \frac{1}{2}$, we have that $1-x \geq e^{-2 x}$. Therefore, we obtain that when $M-2 n_{1} \geq \frac{L_{N}}{2}$ and $n_{2} \leq \frac{L_{N}}{4}$, then the probability that the $n_{1}$ stubs are not connected to the $n_{2}$ stubs when there are still at least $M$ stubs available is bounded below by

$$
\prod_{i=1}^{n_{1}}\left(1-\frac{n_{2}}{M-2 i+1}\right) \geq \prod_{i=1}^{n_{1}} e^{-\frac{4 n_{2}}{L_{N}}}=e^{-\frac{4 n_{1} n_{2}}{L_{N}}} .
$$

The event $H_{N}>i+j$ conditionally on $H_{N}>i+j-1$ precisely occurs when the stubs $Z_{i+1}^{(1, N)}$ are not connected to the stubs in $Z_{j}^{(2, N)}$. We will assume that (4.31) holds. We have that $M=L_{N}-2 \sum_{s=1}^{i+1} Z_{s}^{(1, N)}-2 \sum_{s=1}^{j} Z_{s}^{(2, N)}$, and $n_{1}=Z_{i+1}^{(1, N)}, n_{2}=Z_{j}^{(2, N)}$. Thus, $M-2 n_{1} \geq \frac{L_{N}}{2}$ happens precisely when

$$
M-2 n_{1} \geq L_{N}-2 \sum_{s=1}^{i+1} Z_{s}^{(1, N)}-2 \sum_{s=1}^{j} Z_{s}^{(2, N)} \geq \frac{L_{N}}{2} .
$$


This follows from the assumed bound in (4.31). Also, when $n_{2}=Z_{j}^{(2, N)}, n_{2} \leq \frac{L_{N}}{4}$ is implied by (4.31). Thus, we are allowed to use the bound in (4.37). This leads to

$$
\mathbb{Q}_{Z}^{(i+1, j)}\left(H_{N}>i+j \mid H_{N}>i+j-1\right) \geq \exp \left\{-\frac{4 Z_{i+1}^{(1, N)} Z_{j}^{(2, N)}}{L_{N}}\right\},
$$

which completes the proof of Lemma 4.7

\subsection{The main contribution to $\mathbb{P}\left(\left\{H_{N}>k\right\} \cap E_{m, N}\right)$}

We rewrite the expression in (4.30) in a more convenient form, using Lemma 4.7 We derive an upper and a lower bound. For the upper bound, we bound all terms appearing on the right hand side of (4.20) by 1 , except for the term $\mathbb{Q}_{Z}^{\left(k_{1}+1, k-k_{1}\right)}\left(H_{N}>k \mid H_{N}>k-1\right)$, which arises when $i=k-2 k_{1}$. Using the upper bound in Lemma 4.7, we thus obtain that

$$
P_{m}\left(k, k_{1}\right) \leq \exp \left\{-\frac{Z_{k_{1}+1}^{(1, N)} Z_{k-k_{1}}^{(2, N)}}{2 L_{N}}\right\}
$$

The latter inequality is true for any $Z_{m}$-measurable $k_{1}$ with $m \leq k_{1} \leq(k-1) / 2$.

To derive the lower bound, we next assume that

$$
\sum_{s=1}^{k_{1}+1} Z_{s}^{(1, N)}+\sum_{s=1}^{k-k_{1}} Z_{s}^{(2, N)} \leq \frac{L_{N}}{4}
$$

so that (4.31) is satisfied for all $i$ in (4.20). We write, recalling (3.5),

$$
\mathcal{B}_{N}^{(1)}(\varepsilon, k)=\left\{m \leq l \leq(k-1) / 2: l+1 \in \mathcal{T}_{m}^{(1, N)}(\varepsilon), k-l \in \mathcal{T}_{m}^{(2, N)}(\varepsilon)\right\} .
$$

We restrict ourselves to $k_{1} \in \mathcal{B}_{N}^{(1)}(\varepsilon, k)$, if $\mathcal{B}_{N}^{(1)}(\varepsilon, k) \neq \varnothing$. When $k_{1} \in \mathcal{B}_{N}^{(1)}(\varepsilon, k)$, we are allowed to use the bounds in Proposition 3.2. Note that $\left\{k_{1} \in \mathcal{B}_{N}^{(1)}(\varepsilon, k)\right\}$ is $Z_{m}$-measurable. Moreover, it follows from Proposition 3.2 that if $k_{1} \in \mathcal{B}_{N}^{(1)}(\varepsilon, k)$, that then, with probability converging to 1 as first $N \rightarrow \infty$ and then $m \rightarrow \infty$,

$$
Z_{s}^{(1, N)} \leq N^{\frac{1-\varepsilon^{4}}{\tau-1}}, \quad \forall m<s \leq k_{1}+1, \quad \text { while } \quad Z_{s}^{(2, N)} \leq N^{\frac{1-\varepsilon^{4}}{\tau-1}}, \quad \forall m<s \leq k-k_{1} .
$$

Therefore, when $k_{1} \in \mathcal{B}_{N}^{(1)}(\varepsilon, k)$, the assumption in (4.41) is satisfied with probability $1-o_{N, m}(1)$, as long as $k=O\left(N^{\frac{\tau-2}{\tau-1}}\right)$. The latter restriction is not serious, as we always have $k$ in mind for which $k=O(\log \log N)$ (see e.g. Theorem 1.2).

Thus, on the event $E_{m, N} \cap\left\{k_{1} \in \mathcal{B}_{N}^{(1)}(\varepsilon, k)\right\}$, using (3.7) in Proposition 3.2 and the lower bound in Lemma 4.7 with probability $1-o_{N, m, \varepsilon}(1)$, and for all $i \in\{2 m, \ldots, 2 k-1\}$,

$$
\mathbb{Q}_{Z}^{(\lfloor i / 2\rfloor+1,\lceil i / 2\rceil)}\left(H_{N}>i \mid H_{N}>i-1\right) \geq \exp \left\{-\frac{4 Z_{\lfloor i / 2\rfloor+1}^{(1, N)} Z_{\lceil i / 2\rceil}^{(2, N)}}{L_{N}}\right\} \geq \exp \left\{-\frac{4 Z_{k_{1}+1}^{(1, N)} Z_{k-k_{1}}^{(2, N)}}{L_{N}}\right\},
$$

and, for $1 \leq i \leq k-2 k_{1}$,

$$
\mathbb{Q}_{Z}^{\left(k_{1}+1, k_{1}+i\right)}\left(H_{N}>2 k_{1}+i \mid H_{N}>2 k_{1}+i-1\right) \geq \exp \left\{-\frac{4 Z_{k_{1}+1}^{(1, N)} Z_{k_{1}+i}^{(2, N)}}{L_{N}}\right\} \geq \exp \left\{-\frac{4 Z_{k_{1}+1}^{(1, N)} Z_{k-k_{1}}^{(2, N)}}{L_{N}}\right\} .
$$

Therefore, by Lemma 4.6] and using the above bounds for each of the in total $k$ terms, we obtain that when $k_{1} \in \mathcal{B}_{N}^{(1)}(\varepsilon, k) \neq \varnothing$, and with probability $1-o_{N, m, \varepsilon}(1)$,

$$
P_{m}\left(k, k_{1}\right) \geq \exp \left\{-4 k \frac{Z_{k_{1}+1}^{(1, N)} Z_{k-k_{1}}^{(2, N)}}{L_{N}}\right\} .
$$


We next use the symmetry for the nodes 1 and 2. Denote

$$
\mathcal{B}_{N}^{(2)}(\varepsilon, k)=\left\{m \leq l \leq(k-1) / 2: l+1 \in \mathcal{T}_{m}^{(2, N)}(\varepsilon), k-l \in \mathcal{T}_{m}^{(1, N)}(\varepsilon)\right\} .
$$

Take $\tilde{l}=k-l-1$, so that $(k-1) / 2 \leq \tilde{l} \leq k-1-m$, and thus

$$
\mathcal{B}_{N}^{(2)}(\varepsilon, k)=\left\{(k-1) / 2 \leq \tilde{l} \leq k-1-m: \tilde{l}+1 \in \mathcal{T}_{m}^{(1, N)}(\varepsilon), k-\tilde{l} \in \mathcal{T}_{m}^{(2, N)}(\varepsilon)\right\} .
$$

Then, since the nodes 1 and 2 are exchangeable, we obtain from (4.46), when $k_{1} \in \mathcal{B}_{N}^{(2)}(\varepsilon, k) \neq \varnothing$, and with probability $1-o_{N, m, \varepsilon}(1)$,

$$
P_{m}\left(k, k_{1}\right) \geq \exp \left\{-4 k \frac{Z_{k_{1}+1}^{(1, N)} Z_{k-k_{1}}^{(2, N)}}{L_{N}}\right\} .
$$

We define $\mathcal{B}_{N}(\varepsilon, k)=\mathcal{B}_{N}^{(1)}(\varepsilon, k) \cup \mathcal{B}_{N}^{(2)}(\varepsilon, k)$, which is equal to

$$
\mathcal{B}_{N}(\varepsilon, k)=\left\{m \leq l \leq k-1-m: l+1 \in \mathcal{T}_{m}^{(1, N)}(\varepsilon), k-l \in \mathcal{T}_{m}^{(2, N)}(\varepsilon)\right\} .
$$

We can summarize the obtained results by writing that with probability $1-o_{N, m, \varepsilon}(1)$, and when $\mathcal{B}_{N}(\varepsilon, k) \neq \varnothing$, we have

$$
P_{m}\left(k, k_{1}\right)=\exp \left\{-\lambda_{N} \frac{Z_{k_{1}+1}^{(1, N)} Z_{k-k_{1}}^{(2, N)}}{L_{N}}\right\}
$$

for all $k_{1} \in \mathcal{B}_{N}(\varepsilon, k)$, where $\lambda_{N}=\lambda_{N}(k)$ satisfies

$$
\frac{1}{2} \leq \lambda_{N}(k) \leq 4 k
$$

Relation (4.51) is true for any $k_{1} \in \mathcal{B}_{N}(\varepsilon, k)$. However, our coupling fails when $Z_{k_{1}+1}^{(1, N)}$ or $Z_{k-k_{1}}^{(2, N)}$ grows too large, since we can only couple $Z_{j}^{(i, N)}$ with $\hat{Z}_{j}^{(i, N)}$ up to the point where $Z_{j}^{(i, N)} \leq N^{\frac{1-\varepsilon^{2}}{\tau-1}}$. Therefore, we next take the maximal value over $k_{1} \in \mathcal{B}_{N}(\varepsilon, k)$ to arrive at the fact that, with probability $1-o_{N, m, \varepsilon}(1)$, on the event that $\mathcal{B}_{N}(\varepsilon, k) \neq \varnothing$,

$$
P_{m}\left(k, k_{1}\right)=\max _{k_{1} \in \mathcal{B}_{N}(\varepsilon, k)} \exp \left\{-\lambda_{N} \frac{Z_{k_{1}+1}^{(1, N)} Z_{k-k_{1}}^{(2, N)}}{L_{N}}\right\}=\exp \left\{-\lambda_{N} \min _{k_{1} \in \mathcal{B}_{N}(\varepsilon, k)} \frac{Z_{k_{1}+1}^{(1, N)} Z_{k-k_{1}}^{(2, N)}}{L_{N}}\right\} .
$$

We conclude that

$$
\begin{aligned}
& \mathbb{P}\left(\left\{H_{N}>k\right\} \cap E_{m, N} \cap\left\{\mathcal{B}_{N}(\varepsilon, k) \neq \varnothing\right\}\right) \\
& \quad=\mathbb{E}\left[\mathbf{1}_{E_{m, N}} \exp \left\{-\lambda_{N} \min _{k_{1} \in \mathcal{B}_{N}(\varepsilon, k)} \frac{Z_{k_{1}+1}^{(1, N)} Z_{k-k_{1}}^{(2, N)}}{L_{N}}\right\}\right]+o_{N, m, \varepsilon}(1) .
\end{aligned}
$$

From here on we take $k=k_{N}$ as in (4.4) with $l$ a fixed integer.

In Section 5, we prove the following lemma that shows that, apart from an event of probability $1-o_{N, m, \varepsilon}(1)$, we may assume that $\mathcal{B}_{N}\left(\varepsilon, k_{N}\right) \neq \varnothing$ :

Lemma 4.8 For all $l$, with $k_{N}$ as in 4.4,

$$
\underset{\varepsilon \downarrow 0}{\limsup } \limsup _{m \rightarrow \infty} \limsup _{N \rightarrow \infty} \mathbb{P}\left(\left\{H_{N}>k_{N}\right\} \cap E_{m, N} \cap\left\{\mathcal{B}_{N}\left(\varepsilon, k_{N}\right)=\varnothing\right\}\right)=0 .
$$

From now on, we will abbreviate $\mathcal{B}_{N}=\mathcal{B}_{N}\left(\varepsilon, k_{N}\right)$. Using (4.54) and Lemma 4.8] we conclude Corollary 4.9 For all $l$, with $k_{N}$ as in (4.4),

$$
\mathbb{P}\left(\left\{H_{N}>k_{N}\right\} \cap E_{m, N}\right)=\mathbb{E}\left[\mathbf{1}_{E_{m, N}} \exp \left\{-\lambda_{N} \min _{k_{1} \in \mathcal{B}_{N}} \frac{Z_{k_{1}+1}^{(1, N)} Z_{k-k_{1}}^{(2, N)}}{L_{N}}\right\}\right]+o_{N, m, \varepsilon}(1) .
$$




\subsection{Application of the coupling results}

In this section, we use the coupling results in Section 3.1 Before doing so, we investigate the minimum of the function $t \mapsto \kappa^{t} y_{1}+\kappa^{n-t} y_{2}$, where the minimum is taken over the discrete set $\{0,1, \ldots, n\}$, and we recall that $\kappa=(\tau-2)^{-1}$.

Lemma 4.10 Suppose that $y_{1}>y_{2}>0$, and $\kappa=(\tau-2)^{-1}>1$. For the integer $n>\frac{-\log \left(y_{2} / y_{1}\right)}{\log \kappa}$,

$$
t^{*}=\operatorname{argmin}_{t \in\{0,1, \ldots, n\}}\left(\kappa^{t} y_{1}+\kappa^{n-t} y_{2}\right)=\operatorname{round}\left(\frac{\mathrm{n}}{2}+\frac{\log \left(\mathrm{y}_{2} / \mathrm{y}_{1}\right)}{2 \log \kappa}\right)
$$

where round $(x)$ is $x$ rounded off to the nearest integer. In particular,

$$
\max \left\{\frac{\kappa^{t^{*}} y_{1}}{\kappa^{n-t^{*}} y_{2}}, \frac{\kappa^{n-t^{*}} y_{2}}{\kappa^{t^{*}} y_{1}}\right\} \leq \kappa
$$

Proof. Consider, for real valued $t \in[0, n]$, the function

$$
\psi(t)=\kappa^{t} y_{1}+\kappa^{n-t} y_{2} .
$$

Then,

$$
\psi^{\prime}(t)=\left(\kappa^{t} y_{1}-\kappa^{n-t} y_{2}\right) \log \kappa, \quad \psi^{\prime \prime}(t)=\left(\kappa^{t} y_{1}+\kappa^{n-t} y_{2}\right) \log ^{2} \kappa .
$$

In particular, $\psi^{\prime \prime}(t)>0$, so that the function $\psi$ is strictly convex. The unique minimum of $\psi$ is attained at $\hat{t}$, satisfying $\psi^{\prime}(\hat{t})=0$, i.e.,

$$
\hat{t}=\frac{n}{2}+\frac{\log \left(y_{2} / y_{1}\right)}{2 \log \kappa} \in(0, n)
$$

because $n>-\log \left(y_{2} / y_{1}\right) / \log \kappa$. By convexity $t^{*}=\lfloor\hat{t}\rfloor$ or $t^{*}=\lceil\hat{t}\rceil$. We will show that $\left|t^{*}-\hat{t}\right| \leq \frac{1}{2}$. Put $t_{1}^{*}=\lfloor\hat{t}\rfloor$ and $t_{2}^{*}=\lceil\hat{t}\rceil$. We have

$$
\kappa^{\hat{t}} y_{1}=\kappa^{n-\hat{t}} y_{2}=\kappa^{\frac{n}{2}} \sqrt{y_{1} y_{2}} .
$$

Writing $t_{i}^{*}=\hat{t}+t_{i}^{*}-\hat{t}$, we obtain for $i=1,2$,

$$
\psi\left(t_{i}^{*}\right)=\kappa^{\frac{n}{2}} \sqrt{y_{1} y_{2}}\left\{\kappa^{t_{i}^{*}-\hat{t}}+\kappa^{\hat{t}-t_{i}^{*}}\right\} .
$$

For $0<x<1$, the function $x \mapsto \kappa^{x}+\kappa^{-x}$ is increasing so $\psi\left(t_{1}^{*}\right) \leq \psi\left(t_{2}^{*}\right)$ if and only if $\hat{t}-t_{1}^{*} \leq t_{2}^{*}-\hat{t}$, or $\hat{t}-t_{1}^{*} \leq \frac{1}{2}$, i.e., if $\psi\left(t_{1}^{*}\right) \leq \psi\left(t_{2}^{*}\right)$ and hence the minimum over the discrete set $\{0,1, \ldots, n\}$ is attained at $t_{1}^{*}$, then $\hat{t}-t_{1}^{*} \leq \frac{1}{2}$. On the other hand, if $\psi\left(t_{2}^{*}\right) \leq \psi\left(t_{1}^{*}\right)$, then by the 'only if' statement we find $t_{2}^{*}-\hat{t} \leq \frac{1}{2}$. In both cases we have $\left|t^{*}-\hat{t}\right| \leq \frac{1}{2}$. Finally, if $t^{*}=t_{1}^{*}$, then we obtain, using (4.55),

$$
1 \leq \frac{\kappa^{n-t^{*}} y_{2}}{\kappa^{t^{*}} y_{1}}=\frac{\kappa^{\hat{t}-t_{1}^{*}}}{\kappa^{t_{1}^{*}-\hat{t}}}=\kappa^{2\left(\hat{t}-t_{1}^{*}\right)} \leq \kappa,
$$

while for $t^{*}=t_{2}^{*}$, we obtain $1 \leq \frac{\kappa^{t^{*}} y_{1}}{\kappa^{n-t^{*}} y_{2}} \leq \kappa$.

We continue with our investigation of $\mathbb{P}\left(\left\{H_{N}>k_{N}\right\} \cap E_{m, N}\right)$. We start from Corollary 4.9, substituting (3.3),

$$
\begin{aligned}
& \mathbb{P}\left(\left\{H_{N}>k_{N}\right\} \cap E_{m, N}\right) \\
& =\mathbb{E}\left[\mathbf{1}_{E_{m, N}} \exp \left\{-\lambda_{N} \exp \left[\min _{k_{1} \in \mathcal{B}_{N}}\left(\kappa^{k_{1}+1} Y_{k_{1}+1}^{(1, N)}+\kappa^{k_{N}-k_{1}} Y_{k_{N}-k_{1}}^{(2, N)}\right)-\log L_{N}\right]\right\}\right]+o_{N, m, \varepsilon}(1),
\end{aligned}
$$


where we rewrite, using (4.50) and (3.5),

$$
\mathcal{B}_{N}=\left\{m \leq k_{1} \leq k_{N}-1-m: \kappa^{k_{1}+1} Y_{m}^{(1, N)} \leq \frac{1-\varepsilon^{2}}{\tau-1} \log N, \kappa^{k_{N}-k_{1}} Y_{m}^{(2, N)} \leq \frac{1-\varepsilon^{2}}{\tau-1} \log N\right\} .
$$

Moreover, according to Proposition [3.2, and with probability at least $1-o_{N, m, \varepsilon}(1)$, we have that $\min _{k_{1} \in \mathcal{B}_{N}}\left(\kappa^{k_{1}+1} Y_{k_{1}+1}^{(1, N)}+\kappa^{k_{N}-k_{1}} Y_{k_{N}-k_{1}}^{(2, N)}\right)$ is between

$$
\min _{k_{1} \in \mathcal{B}_{N}}\left(\kappa^{k_{1}+1}\left(Y_{m}^{(1, N)}-\varepsilon^{3}\right)+\kappa^{k_{N}-k_{1}}\left(Y_{m}^{(2, N)}-\varepsilon^{3}\right)\right)
$$

and

$$
\min _{k_{1} \in \mathcal{B}_{N}}\left(\kappa^{k_{1}+1}\left(Y_{m}^{(1, N)}+\varepsilon^{3}\right)+\kappa^{k_{N}-k_{1}}\left(Y_{m}^{(2, N)}+\varepsilon^{3}\right)\right) .
$$

To abbreviate the notation, we will write, for $i=1,2$,

$$
Y_{m,+}^{(i, N)}=Y_{m}^{(i, N)}+\varepsilon^{3}, \quad Y_{m,-}^{(i, N)}=Y_{m}^{(i, N)}-\varepsilon^{3} .
$$

Define for $\varepsilon>0$,

$$
H_{m, N}=H_{m, N}(\varepsilon)=\left\{\min _{0 \leq k_{1} \leq k_{N}-1}\left(\kappa^{k_{1}+1} Y_{m,-}^{(1, N)}+\kappa^{k_{N}-k_{1}} Y_{m,-}^{(2, N)}\right) \leq\left(1+\varepsilon^{2}\right) \log N\right\} .
$$

On the complement $H_{m, N}^{c}$, the minimum over $0 \leq k_{1} \leq k_{N}-1$ of $\kappa^{k_{1}+1} Y_{m,-}^{(1, N)}+\kappa^{k_{N}-k_{1}} Y_{m,-}^{(2, N)}$ exceeds $\left(1+\varepsilon^{2}\right) \log N$. Therefore, also the minimum over the set $\mathcal{B}_{N}$ of $\kappa^{k_{1}+1} Y_{m,-}^{(1, N)}+\kappa^{k_{N}-k_{1}} Y_{m,-}^{(2, N)}$ exceeds $\left(1+\varepsilon^{2}\right) \log N$, so that, using Lemma 4.7 and with error at most $o_{N, m, \varepsilon}(1)$,

$$
\begin{aligned}
\mathbb{P}\left(\left\{H_{N}>k_{N}\right\} \cap E_{m, N} \cap H_{m, N}^{c}\right) \\
\leq \mathbb{E}\left[\mathbf{1}_{H_{m, N}^{c}} \exp \left\{-\frac{1}{2} \exp \left[\min _{k_{1} \in \mathcal{B}_{N}}\left(\kappa^{k_{1}+1} Y_{k_{1}+1}^{(1, N)}+\kappa^{k_{N}-k_{1}} Y_{k_{N}-k_{1}}^{(2, N)}\right)-\log L_{N}\right]\right\}\right] \\
\leq \mathbb{E}\left[\mathbf{1}_{H_{m, N}^{c}} \exp \left\{-\frac{1}{2} \exp \left[\min _{k_{1} \in \mathcal{B}_{N}}\left(\kappa^{k_{1}+1} Y_{m,-}^{(1, N)}+\kappa^{k_{N}-k_{1}} Y_{m,-}^{(2, N)}\right)-\log L_{N}\right]\right\}\right] \\
\leq \mathbb{E}\left[\exp \left\{-\frac{1}{2} \exp \left(\left(1+\varepsilon^{2}\right) \log N-\log L_{N}\right)\right\}\right] \leq e^{-\frac{1}{2 c} N^{\varepsilon^{2}}},
\end{aligned}
$$

because $L_{N} \leq c N$, whp, as $N \rightarrow \infty$. Therefore, in the remainder of the proof, we assume that $H_{m, N}$ holds.

We next show that whp ,

$$
\min _{k_{1} \in \mathcal{B}_{N}}\left(\kappa^{k_{1}+1} Y_{m,+}^{(1, N)}+\kappa^{k_{N}-k_{1}} Y_{m,+}^{(2, N)}\right)=\min _{0 \leq k_{1}<k_{N}}\left(\kappa^{k_{1}+1} Y_{m,+}^{(1, N)}+\kappa^{k_{N}-k_{1}} Y_{m,+}^{(2, N)}\right)
$$

and

$$
\min _{k_{1} \in \mathcal{B}_{N}}\left(\kappa^{k_{1}+1} Y_{m,-}^{(1, N)}+\kappa^{k_{N}-k_{1}} Y_{m,-}^{(2, N)}\right)=\min _{0 \leq k_{1}<k_{N}}\left(\kappa^{k_{1}+1} Y_{m,-}^{(1, N)}+\kappa^{k_{N}-k_{1}} Y_{m,-}^{(2, N)}\right)
$$

We start with (4.60), the proof of (4.61) is similar, and, in fact, slightly simpler, and is therefore omitted. To prove (4.60), we use Lemma 4.10, with $n=k_{N}+1, t=k_{1}+1, y_{1}=Y_{m,+}^{(1, N)}$ and $y_{2}=Y_{m,+}^{(2, N)}$. Let

$$
t^{*}=\operatorname{argmin}_{t \in\{0,1, \ldots, n\}}\left(\kappa^{t} y_{1}+\kappa^{n-t} y_{2}\right),
$$

and assume (without restriction) that $\kappa^{t^{*}} y_{1} \geq \kappa^{n-t^{*}} y_{2}$. We have to show that $t^{*}-1 \in \mathcal{B}_{N}$. According to Lemma 4.10.

$$
1 \leq \frac{\kappa^{t^{*}} Y_{m,+}^{(1, N)}}{\kappa^{n-t^{*}} Y_{m,+}^{(2, N)}}=\frac{\kappa^{t^{*}} y_{1}}{\kappa^{n-t^{*}} y_{2}} \leq \kappa
$$


We define $x=\kappa^{t^{*}} Y_{m,+}^{(1, N)}$ and $y=\kappa^{n-t^{*}} Y_{m,+}^{(2, N)}$, so that $x \geq y$. By definition, on $H_{m, N}(\varepsilon)$,

$$
\kappa^{t^{*}} Y_{m,-}^{(1, N)}+\kappa^{n-t^{*}} Y_{m,-}^{(2, N)} \leq\left(1+\varepsilon^{2}\right) \log N
$$

Since, on $E_{m, N}$, we have that $Y_{m}^{(1, N)} \geq \varepsilon$,

$$
Y_{m,+}^{(1, N)} \leq \frac{\varepsilon+\varepsilon^{3}}{\varepsilon-\varepsilon^{3}} Y_{m,-}^{(1, N)}=\frac{1+\varepsilon^{2}}{1-\varepsilon^{2}} Y_{m,-}^{(1, N)}
$$

and likewise for $Y_{m,+}^{(2, N)}$. Therefore, we obtain that on $E_{m, N} \cap F_{m, N} \cap H_{m, N}$, and with $\varepsilon$ sufficiently small,

$$
x+y \leq \frac{1+\varepsilon^{2}}{1-\varepsilon^{2}}\left[\kappa^{t^{*}} Y_{m,-}^{(1, N)}+\kappa^{n-t^{*}} Y_{m,-}^{(2, N)}\right] \leq \frac{\left(1+\varepsilon^{2}\right)^{2}}{1-\varepsilon^{2}} \log N \leq(1+\varepsilon) \log N .
$$

Moreover, by (4.62), we have that

$$
1 \leq \frac{x}{y} \leq \kappa
$$

Hence, on $E_{m, N} \cap F_{m, N} \cap H_{m, N}$, we have

$$
x=\frac{x+y}{1+\frac{y}{x}} \leq(1+\varepsilon) \frac{1}{1+\kappa^{-1}} \log N=\frac{1+\varepsilon}{\tau-1} \log N,
$$

when $\varepsilon>0$ is sufficiently small. We claim that if

$$
x=\kappa^{t^{*}} Y_{m,+}^{(1, N)} \leq \frac{1-\varepsilon}{\tau-1} \log N,
$$

then $k^{*}=t^{*}-1 \in \mathcal{B}_{N}\left(\varepsilon, k_{N}\right)$, so that (4.60) follows. Indeed, we use (4.67) to see that

$$
\kappa^{k^{*}+1} Y_{m}^{(1, N)}=\kappa^{t^{*}} Y_{m}^{(1, N)} \leq \kappa^{t^{*}} Y_{m,+}^{(1, N)} \leq \frac{1-\varepsilon}{\tau-1} \log N
$$

so that the first bound in (4.57) is satisfied. The second bound is satisfied, since

$$
\kappa^{k_{N}-k^{*}} Y_{m}^{(2, N)}=\kappa^{n-t^{*}} Y_{m}^{(2, N)} \leq \kappa^{n-t^{*}} Y_{m,+}^{(2, N)}=y \leq x \leq \frac{1-\varepsilon}{\tau-1} \log N,
$$

where we have used $n=k_{N}+1$, and (4.67).

Thus, in order to show that (4.60) holds with probability close to 1 , we have to show that the probability of the intersection of the events $\left\{H_{N}>k_{N}\right\}$ and

$$
\begin{array}{r}
\mathcal{E}_{m, N}=\mathcal{E}_{m, N}(\varepsilon)=\left\{\exists t: \frac{1-\varepsilon}{\tau-1} \log N<\kappa^{t} Y_{m,+}^{(1, N)} \leq \frac{1+\varepsilon}{\tau-1} \log N,\right. \\
\left.\kappa^{t} Y_{m,+}^{(1, N)}+\kappa^{n-t} Y_{m,+}^{(2, N)} \leq(1+\varepsilon) \log N\right\},
\end{array}
$$

can be made arbitrarily small by choosing $\varepsilon$ close to 0 , when first $N \rightarrow \infty$ and then $m \rightarrow \infty$. That is the content of the following lemma, whose proof is deferred to Section 5

Lemma 4.11 For $k_{N}$ as in 4.4,

$$
\limsup _{\varepsilon \downarrow 0} \limsup _{m \rightarrow \infty} \limsup _{N \rightarrow \infty} \mathbb{P}\left(E_{m, N}(\varepsilon) \cap \mathcal{E}_{m, N}(\varepsilon) \cap\left\{H_{N}>k_{N}\right\}\right)=0 .
$$

Therefore, we finally arrive at

$$
\begin{aligned}
& \mathbb{P}\left(\left\{H_{N}>k_{N}\right\} \cap E_{m, N}\right) \\
& \quad \leq \mathbb{E}\left[\mathbf{1}_{E_{m, N}} \exp \left\{-\lambda_{N} \exp \left[\min _{0 \leq k_{1}<k_{N}}\left(\kappa^{k_{1}+1} Y_{m,+}^{(1, N)}+\kappa^{k_{N}-k_{1}} Y_{m,+}^{(2, N)}\right)-\log L_{N}\right]\right\}\right]+o_{N, m, \varepsilon}(1)
\end{aligned}
$$

and at a similar lower bound where $Y_{m,+}^{(i, N)}$ is replaced by $Y_{m,-}^{(i, N)}$. 


\subsection{Evaluating the limit}

The final argument consists of letting $N \rightarrow \infty$ and then $m \rightarrow \infty$. The argument has to be performed with $Y_{m,+}^{(i, N)}$ and $Y_{m,-}^{(1, N)}$ separately, after which we let $\varepsilon \downarrow 0$. Since the precise value of $\varepsilon$ plays no role in the derivation, we only give the derivation for $\varepsilon=0$. Observe that

$$
\begin{aligned}
& \min _{0 \leq k_{1}<k_{N}}\left(\kappa^{k_{1}+1} Y_{m}^{(1, N)}+\kappa^{k_{N}-k_{1}} Y_{m}^{(2, N)}\right)-\log L_{N} \\
& =\kappa^{\left\lceil k_{N} / 2\right\rceil} \min _{0 \leq k_{1}<k_{N}}\left(\kappa^{k_{1}+1-\left\lceil k_{N} / 2\right\rceil} Y_{m}^{(1, N)}+\kappa^{\left\lfloor k_{N} / 2\right\rfloor-k_{1}} Y_{m}^{(2, N)}-\kappa^{-\left\lceil k_{N} / 2\right\rceil} \log L_{N}\right) \\
& \left.=\kappa^{\left\lceil k_{N} / 2\right\rceil} \quad-\quad \min _{N} / 2\right\rceil+1 \leq t<\left\lfloor k_{N} / 2\right\rfloor+1 \\
&
\end{aligned}
$$

where $t=k_{1}+1-\left\lceil k_{N} / 2\right\rceil, c_{k_{N}}=c_{l}=\lfloor l / 2\rfloor-\lceil l / 2\rceil+1=1_{\{l \text { is even }\}}$. We further rewrite, using (4.4) and the definition of $a_{N}$ in Theorem 1.2.

$$
\kappa^{-\left\lceil k_{N} / 2\right\rceil} \log L_{N}=\kappa^{\frac{\log \log N}{\log \kappa}-\left\lfloor\frac{\log \log N}{\log \kappa}\right\rfloor-\lceil l / 2\rceil} \frac{\log L_{N}}{\log N}=\kappa^{-a_{N}-\lceil l / 2\rceil} \frac{\log L_{N}}{\log N} .
$$

From Lemma 4.10, for $N \rightarrow \infty$ and on the event $E_{m, N}$,

$$
\min _{-\left\lceil k_{N} / 2\right\rceil+1 \leq t \leq\left\lfloor k_{N} / 2\right\rfloor}\left(\kappa^{t} Y_{m}^{(1, N)}+\kappa^{c_{l}-t} Y_{m}^{(2, N)}\right)=\min _{t \in \mathbb{Z}}\left(\kappa^{t} Y_{m}^{(1, N)}+\kappa^{c_{l}-t} Y_{m}^{(2, N)}\right),
$$

because $Y_{m}^{(i, N)} \in\left[\varepsilon, \varepsilon^{-1}\right]$ on $E_{m, N}$. We define

$$
W_{m, N}\left(k_{N}\right)=\min _{t \in \mathbb{Z}}\left(\kappa^{t} Y_{m}^{(1, N)}+\kappa^{c_{l}-t} Y_{m}^{(2, N)}\right)-\kappa^{-a_{N}-\lceil l / 2\rceil} \frac{\log L_{N}}{\log N},
$$

and, for $\varepsilon>0$,

$$
\widetilde{F}_{N}=\widetilde{F}_{N}(l, \varepsilon)=\left\{W_{m, N}\left(k_{N}\right)>\varepsilon\right\}, \quad \widetilde{G}_{N}=\widetilde{G}_{N}(l, \varepsilon)=\left\{W_{m, N}\left(k_{N}\right)<-\varepsilon\right\} .
$$

Observe that

$$
\kappa^{\left\lceil k_{N} / 2\right\rceil} W_{m, N}\left(k_{N}\right) \cdot \mathbf{1}_{\widetilde{F}_{N}} \geq \kappa^{\left\lceil k_{N} / 2\right\rceil} \varepsilon, \quad \quad \kappa^{\left\lceil k_{N} / 2\right\rceil} W_{m, N}\left(k_{N}\right) \cdot \mathbf{1}_{\widetilde{G}_{N}} \leq \kappa^{\left\lceil k_{N} / 2\right\rceil}(-\varepsilon) .
$$

We split

$$
\begin{aligned}
\mathbb{P}\left(\left\{H_{N}>k_{N}\right\} \cap E_{m, N}\right) & =\mathbb{E}\left[\mathbf{1}_{E_{m, N}} \exp \left[-\lambda_{N} e^{\left.\left.\kappa \kappa_{N} / 2\right\rceil W_{m, N}\left(k_{N}\right)\right]}\right]+o_{N, m, \varepsilon}(1)\right. \\
& =\mathbb{P}\left(\widetilde{G}_{N} \cap E_{m, N}\right)+I_{N}+J_{N}+K_{N}+o_{N, m, \varepsilon}(1),
\end{aligned}
$$

where we define

$$
\begin{aligned}
I_{N} & =\mathbb{E}\left[\exp \left[-\lambda_{N} e^{\kappa^{\left\lceil k_{N} / 2\right\rceil} W_{m, N}\left(k_{N}\right)}\right] \mathbf{1}_{\widetilde{F}_{N} \cap E_{m, N}}\right], \\
J_{N} & =\mathbb{E}\left[\left(\exp \left[-\lambda_{N} e^{\kappa^{\left\lceil k_{N} / 2\right\rceil} W_{m, N}\left(k_{N}\right)}\right]-1\right) \mathbf{1}_{\widetilde{G}_{N} \cap E_{m, N}}\right], \\
K_{N} & =\mathbb{E}\left[\exp \left[-\lambda_{N} e^{\kappa^{\left\lceil k_{N} / 2\right\rceil} W_{m, N}\left(k_{N}\right)}\right] \mathbf{1}_{\widetilde{F}_{N}^{c} \cap \widetilde{G}_{N}^{c} \cap E_{m, N}}\right] .
\end{aligned}
$$

We first show that $I_{N}, J_{N}$ and $K_{N}$ are error terms, and then prove convergence of $\mathbb{P}\left(\widetilde{G}_{N} \cap E_{m, N}\right)$.

We start by bounding $I_{N}$. By the first bound in (4.77), for every $\varepsilon>0$, and since $\lambda_{N} \geq \frac{1}{2}$,

$$
\limsup _{N \rightarrow \infty} I_{N} \leq \limsup _{N \rightarrow \infty} \exp \left\{-\frac{1}{2} \exp \left\{\kappa^{\left\lceil k_{N} / 2\right\rceil} \varepsilon\right\}\right\}=0 .
$$


Similarly, by the second bound in (4.77), for every $\varepsilon>0$, and since $\lambda_{N} \leq 4 k_{N}$, we can bound $J_{N}$ as

$$
\limsup _{N \rightarrow \infty}\left|J_{N}\right| \leq \limsup _{N \rightarrow \infty} \mathbb{E}\left[1-\exp \left\{-4 k_{N} \exp \left\{-\kappa^{\left\lceil k_{N} / 2\right\rceil} \varepsilon\right\}\right\}\right]=0 .
$$

Finally, we bound $K_{N}$ by

$$
K_{N} \leq \mathbb{P}\left(\widetilde{F}_{N}^{c} \cap \widetilde{G}_{N}^{c} \cap E_{m, N}\right)
$$

We will next show that

$$
\mathbb{P}\left(\widetilde{G}_{N} \cap E_{m, N}\right)=\mathbb{P}\left(\min _{t \in \mathbb{Z}}\left(\kappa^{t} Y^{(1)}+\kappa^{c_{l}-t} Y^{(2)}\right)-\kappa^{-a_{N}-\lceil l / 2\rceil} \leq 0, Y^{(1)} Y^{(2)}>0\right)+o_{N, m, \varepsilon}(1),
$$

and

$$
\mathbb{P}\left(\widetilde{F}_{N}^{c} \cap \widetilde{G}_{N}^{c} \cap E_{m, N}\right)=o_{N, m, \varepsilon}(1) .
$$

Equation (4.86) is the content of the following lemma, whose proof is deferred to Section 5 ;

Lemma 4.12 For all $l$,

$$
\limsup _{\varepsilon \downarrow 0} \limsup _{m \rightarrow \infty} \limsup _{N \rightarrow \infty} \mathbb{P}\left(\widetilde{F}_{N}(l, \varepsilon)^{c} \cap \widetilde{G}_{N}(l, \varepsilon)^{c} \cap E_{m, N}(\varepsilon)\right)=0 .
$$

We now prove (4.85). From the definition of $\widetilde{G}_{N}$,

$$
\widetilde{G}_{N} \cap E_{m, N}=\left\{\min _{t \in \mathbb{Z}}\left(\kappa^{t} Y_{m}^{(1, N)}+\kappa^{c_{l}-t} Y_{m}^{(2, N)}\right)-\kappa^{-a_{N}-\lceil l / 2\rceil} \frac{\log L_{N}}{\log N}<-\varepsilon, Y_{m}^{(i, N)} \in\left[\varepsilon, \varepsilon^{-1}\right]\right\} .
$$

By Proposition 3.1 and the fact that $L_{N}=\mu N(1+o(1))$ with probability $1-o_{N}(1)$,

$$
\mathbb{P}\left(\widetilde{G}_{N} \cap E_{m, N}\right)=\mathbb{P}\left(\min _{t \in \mathbb{Z}}\left(\kappa^{t} Y_{m}^{(1)}+\kappa^{c_{l}-t} Y_{m}^{(2)}\right)-\kappa^{-a_{N}-\lceil l / 2\rceil}<-\varepsilon, Y_{m}^{(i)} \in\left[\varepsilon, \varepsilon^{-1}\right]\right)+o_{N}(1) .
$$

Since $Y_{m}^{(i)}$ converges to $Y^{(i)}$ almost surely, $\sup _{s \geq m}\left|Y_{s}^{(i)}-Y^{(i)}\right|$ converges to 0 a.s. as $m \rightarrow \infty$. Therefore,

$$
\mathbb{P}\left(\widetilde{G}_{N} \cap E_{m, N}\right)=\mathbb{P}\left(M_{l}-\kappa^{-a_{N}-\lceil l / 2\rceil}<-\varepsilon, Y^{(i)} \in\left[\varepsilon, \varepsilon^{-1}\right]\right)+o_{N, m, \varepsilon}(1),
$$

where we define

$$
M_{l}=\min _{t \in \mathbb{Z}}\left(\kappa^{t} Y^{(1)}+\kappa^{c_{l}-t} Y^{(2)}\right) .
$$

Moreover, since $Y^{(1)}$ has a density on $(0, \infty)$ and an atom at 0 (see [16]),

$$
\mathbb{P}\left(Y^{(1)} \notin\left[\varepsilon, \varepsilon^{-1}\right], Y^{(1)}>0\right)=o_{\varepsilon}(1),
$$

so that, in turn,

$$
\begin{aligned}
\mathbb{P}\left(\widetilde{G}_{N} \cap E_{m, N}\right) & =\mathbb{P}\left(M_{l}-\kappa^{-a_{N}-\lceil l / 2\rceil}<-\varepsilon, Y^{(1)} Y^{(2)}>0\right)+o_{N, m, \varepsilon}(1) \\
& =q^{2} \mathbb{P}\left(M_{l}-\kappa^{-a_{N}-\lceil l / 2\rceil}<-\varepsilon \mid Y^{(1)} Y^{(2)}>0\right)+o_{N, m, \varepsilon}(1) .
\end{aligned}
$$

Recall from Section 2 that for any $l$ fixed, conditionally on $Y^{(1)} Y^{(2)}>0$, the random variable $M_{l}$ has a density. We denote this density by $f_{2}$ and the distribution function by $F_{2}$. Also, $\kappa^{-a_{N}-\lceil l / 2\rceil} \in$ $I_{l}=\left[\kappa^{-\lceil l / 2\rceil}, \kappa^{-\lceil l / 2\rceil+1}\right]$. Then,

$$
\mathbb{P}\left(-\varepsilon \leq M_{l}-\kappa^{-a_{N}-\lceil l / 2\rceil}<0\right) \leq \sup _{a \in I_{l}}\left[F_{2}(a)-F_{2}(a-\varepsilon)\right] .
$$

The function $F_{2}$ is continuous on $I_{l}$, so that in fact $F_{2}$ is uniformly continuous on $I_{l}$, and we conclude that

$$
\limsup _{\varepsilon \downarrow 0} \sup _{a \in I_{l}}\left[F_{2}(a)-F_{2}(a-\varepsilon)\right]=0 .
$$

We conclude the results of Sections 4.2 4.6 in the following corollary: 
Corollary 4.13 For all $l$, with $k_{N}$ as in 4.4),

$$
\mathbb{P}\left(\left\{H_{N}>k_{N}\right\} \cap\left\{Y^{(1)} Y^{(2)}>0\right\}\right)=q^{2} \mathbb{P}\left(M_{l}-\kappa^{-a_{N}-\lceil l / 2\rceil} \leq 0 \mid Y^{(1)} Y^{(2)}>0\right)+o_{N, m, \varepsilon}(1) .
$$

We now come to the conclusion of the proofs of Theorems 1.2 and 1.5 We combine the results in Corollaries 4.4 and 4.13, together with the fact that $q_{m}=q+o(1)$ as $m \rightarrow \infty$, to obtain that, with $k_{\tau, N}=2\left\lfloor\frac{\log \log N}{|\log (\tau-2)|}\right\rfloor$,

$$
\mathbb{P}\left(H_{N}>k_{\tau, N}+l\right)=1-q^{2}+q^{2} \mathbb{P}\left(M_{l}-\kappa^{-a_{N}-\lceil l / 2\rceil} \leq 0 \mid Y^{(1)} Y^{(2)}>0\right)+o_{N, m, \varepsilon}(1) .
$$

Therefore,

$$
\mathbb{P}\left(H_{N} \leq k_{\tau, N}+l\right)=q^{2} \mathbb{P}\left(\min _{t \in \mathbb{Z}}\left(M_{l}>\kappa^{-a_{N}-\lceil l / 2\rceil} \mid Y^{(1)} Y^{(2)}>0\right)+o_{N, m, \varepsilon}(1) .\right.
$$

When $l \rightarrow \infty$, we claim that 4.95) implies that, when $N \rightarrow \infty$,

$$
\mathbb{P}\left(H_{N}<\infty\right)=q^{2}+o(1) .
$$

Indeed, to see (4.96), we prove upper and lower bounds. For the lower bound, we use that for any $l \in \mathbb{Z}$

$$
\mathbb{P}\left(H_{N}<\infty\right) \geq \mathbb{P}\left(H_{N} \leq k_{\tau, N}+l\right),
$$

and let $l \rightarrow \infty$ in (4.95), together with the fact that $\kappa^{-a_{N}-\lceil l / 2\rceil} \rightarrow 0$ as $l \rightarrow \infty$. For the upper bound, we split

$$
\mathbb{P}\left(H_{N}<\infty\right)=\mathbb{P}\left(\left\{H_{N}<\infty\right\} \cap\left\{Y_{m}^{(1, N)} Y_{m}^{(2, N)}=0\right\}\right)+\mathbb{P}\left(\left\{H_{N}<\infty\right\} \cap\left\{Y_{m}^{(1, N)} Y_{m}^{(2, N)}>0\right\}\right) .
$$

The first term is bounded by $\mathbb{P}\left(H_{N} \leq m-1\right)=o_{N}(1)$, by Lemma 4.3. The second term is bounded from above by, using Proposition 3.1 .

$$
\mathbb{P}\left(\left\{H_{N}<\infty\right\} \cap\left\{Y_{m}^{(1, N)} Y_{m}^{(2, N)}>0\right\}\right) \leq \mathbb{P}\left(Y_{m}^{(1, N)} Y_{m}^{(2, N)}>0\right)=q_{m}^{2}+o_{N}(1),
$$

which converges to $q^{2}$ as $m \rightarrow \infty$. This proves (4.96). We conclude from (4.95) and (4.96) that

$$
\mathbb{P}\left(H_{N} \leq k_{\tau, N}+l \mid H_{N}<\infty\right)=\mathbb{P}\left(M_{l} \geq(\tau-2)^{a_{N}+\lceil l / 2\rceil} \mid Y^{(1)} Y^{(2)}>0\right)+o(1) .
$$

This completes the proofs of Theorems 1.2 and 1.5 ,

\section{Proofs of Lemmas $4.8,4.11$ and 4.12}

In this section, we prove the three lemmas used in Section 4 The proofs are similar in nature. Denote

$$
\left\{k \in \partial \mathcal{T}_{m}^{(N)}\right\}=\left\{k \in \mathcal{T}_{m}^{(N)}\right\} \cap\left\{k+1 \notin \mathcal{T}_{m}^{(N)}\right\} .
$$

We will make essential use of the following consequence of Propositions 3.1 and 3.2 .

Lemma 5.1 Assume that Propositions [3.1 and 3.2 hold. Then, for any $u>0$, and $i=1,2$,

$$
\mathbb{P}\left(\left\{k \in \mathcal{T}_{m}^{(i, N)}\right\} \cap\left\{\varepsilon \leq Y_{m}^{(i, N)} \leq \varepsilon^{-1}\right\} \cap\left\{Z_{k}^{(i, N)} \in\left[N^{u(1-\varepsilon)}, N^{u(1+\varepsilon)}\right]\right\}\right)=o_{N, m, \varepsilon}(1) .
$$

Consequently,

$$
\mathbb{P}\left(\left\{k \in \partial \mathcal{T}_{m}^{(i, N)}\right\} \cap\left\{\varepsilon \leq Y_{m}^{(i, N)} \leq \varepsilon^{-1}\right\} \cap\left\{Z_{k}^{(i, N)} \leq N^{\frac{1}{\kappa(\tau-1)}+\varepsilon}\right\}\right)=o_{N, m, \varepsilon}(1) .
$$




\section{Proof. By Proposition 3.2 whp}

$$
Y_{k}^{(i, N)} \leq Y_{m}^{(i, N)}+\varepsilon^{3} \leq Y_{m}^{(i, N)}\left(1+\varepsilon^{2}\right),
$$

where the last inequality follows from $Y_{m}^{(i, N)} \leq \varepsilon^{-1}$. Therefore, also

$$
Y_{m}^{(i, N)} \geq Y_{k}^{(i, N)}\left(1-2 \varepsilon^{2}\right),
$$

when $\varepsilon$ is so small that $\left(1+\varepsilon^{2}\right)^{-1} \geq 1-2 \varepsilon^{2}$. In a similar way, we conclude that

$$
Y_{m}^{(i, N)} \leq Y_{k}^{(i, N)}\left(1+2 \varepsilon^{2}\right) .
$$

Furthermore, the event $Z_{k}^{(i, N)} \in\left[N^{u(1-\varepsilon)}, N^{u(1+\varepsilon)}\right]$ is equivalent to

$$
(1-\varepsilon) u \kappa^{-k} \log N \leq Y_{k}^{(i, N)} \leq(1+\varepsilon) u \kappa^{-k} \log N .
$$

Therefore, we obtain that, with $u_{k, N}=u \kappa^{-k} \log N$,

$$
Y_{m}^{(i, N)} \leq\left(1+2 \varepsilon^{2}\right)(1+\varepsilon) u \kappa^{-k} \log N \leq(1+2 \varepsilon) u_{k, N} .
$$

Similarly, we obtain

$$
Y_{m}^{(i, N)} \geq\left(1-2 \varepsilon^{2}\right)(1-\varepsilon) u \kappa^{-k} \log N \geq(1-2 \varepsilon) u_{k, N} .
$$

We conclude that the events $k \in \mathcal{T}_{m}^{(i, N)}, \varepsilon \leq Y_{m}^{(i, N)} \leq \varepsilon^{-1}$ and $Z_{k}^{(i, N)} \in\left[N^{u(1-\varepsilon)}, N^{u(1+\varepsilon)}\right]$ imply

$$
Y_{m}^{(i, N)} \in u_{k, N}[1-2 \varepsilon, 1+2 \varepsilon] \equiv\left[u_{k, N}(1-2 \varepsilon), u_{k, N}(1+2 \varepsilon)\right] .
$$

Since $\varepsilon \leq Y_{m}^{(N)} \leq \varepsilon^{-1}$, we therefore must also have (when $\varepsilon$ is so small that $1-2 \varepsilon \geq \frac{1}{2}$ ),

$$
u_{k, N} \in\left[\frac{\varepsilon}{2}, \frac{2}{\varepsilon}\right]
$$

Therefore,

$$
\begin{aligned}
& \limsup \limsup _{\varepsilon \downarrow 0} \limsup _{m \rightarrow \infty} \mathbb{P}\left(\left\{k \in \mathcal{T}_{m}^{(i, N)}\right\} \cap\left\{\varepsilon \leq Y_{m}^{(i, N)} \leq \varepsilon^{-1}\right\} \cap\left\{Z_{k}^{(i, N)} \in\left[N^{u(1-\varepsilon)}, N^{u(1+\varepsilon)}\right]\right\}\right) \\
& \quad \leq \limsup _{\varepsilon \downarrow 0} \limsup _{m \rightarrow \infty} \limsup _{N \rightarrow \infty} \sup _{x \in\left[\frac{\varepsilon}{2}, \frac{2}{\varepsilon}\right]} \mathbb{P}\left(Y_{m}^{(i, N)} \in x[1-2 \varepsilon, 1+2 \varepsilon]\right) .
\end{aligned}
$$

Since $Y_{m}^{(i, N)}=Y_{m}^{(i)}$ whp by Proposition 3.1, we arrive at

$$
\begin{aligned}
& \underset{\varepsilon \downarrow 0}{\limsup } \limsup _{m \rightarrow \infty} \limsup _{N \rightarrow \infty} \mathbb{P}\left(\left\{k \in \mathcal{T}_{m}^{(i, N)}\right\} \cap\left\{\varepsilon \leq Y_{m}^{(i, N)} \leq \varepsilon^{-1}\right\} \cap\left\{Z_{k}^{(i, N)} \in\left[N^{u(1-\varepsilon)}, N^{u(1+\varepsilon)}\right]\right\}\right) \\
& \quad \leq \limsup _{\varepsilon \downarrow 0} \limsup _{m \rightarrow \infty} \sup _{x \in\left[\frac{\varepsilon}{2}, \frac{2}{\varepsilon}\right]} \mathbb{P}\left(Y_{m}^{(i)} \in x[1-2 \varepsilon, 1+2 \varepsilon]\right) .
\end{aligned}
$$

We next use that $Y_{m}^{(i)}$ converges to $Y^{(i)}$ almost surely as $m \rightarrow \infty$ to arrive at

$$
\begin{aligned}
& \limsup \limsup _{\varepsilon \downarrow 0} \limsup _{m \rightarrow \infty} \mathbb{P}\left(\left\{k \in \mathcal{T}_{m}^{(i, N)}\right\} \cap\left\{\varepsilon \leq Y_{m}^{(i, N)} \leq \varepsilon^{-1}\right\} \cap\left\{Z_{k}^{(i, N)} \in\left[N^{u(1-\varepsilon)}, N^{u(1+\varepsilon)}\right]\right\}\right) \\
& \quad \leq \underset{\varepsilon \downarrow 0}{\limsup } \sup _{x \in\left[\frac{\varepsilon}{2}, \frac{2}{\varepsilon}\right]} \mathbb{P}\left(Y^{(i)} \in x[1-2 \varepsilon, 1+2 \varepsilon]\right) \leq \limsup _{\varepsilon \downarrow 0} \sup _{x>0}\left[F_{1}(x(1+2 \varepsilon))-F_{1}(x(1-2 \varepsilon))\right],
\end{aligned}
$$

where $F_{1}$ denotes the distribution function of $Y^{(i)}$, which is continuous for $x>0$. Moreover,

$$
\lim _{x \rightarrow \infty} 1-F_{1}(x)=0 .
$$


Therefore, uniformly in $\varepsilon<1 / 4$,

$$
\sup _{x>K}\left[F_{1}(x(1+2 \varepsilon))-F_{1}(x(1-2 \varepsilon))\right] \leq 2 \sup _{x>K}\left[1-F_{1}(x(1-2 \varepsilon))\right] \rightarrow 0, \quad(K \rightarrow \infty),
$$

so that

$$
\begin{aligned}
\sup _{x>0}[ & \left.F_{1}(x(1+2 \varepsilon))-F_{1}(x(1-2 \varepsilon))\right] \\
& \leq \sup _{0<x \leq K}\left[F_{1}(x(1+2 \varepsilon))-F_{1}(x(1-2 \varepsilon))\right]+\sup _{x>K}\left[F_{1}(x(1+2 \varepsilon))-F_{1}(x(1-2 \varepsilon))\right] \\
& =\sup _{0<x \leq K}\left[F_{1}(x(1+2 \varepsilon))-F_{1}(x(1-2 \varepsilon))\right]+o(1) .
\end{aligned}
$$

Therefore,

$\limsup _{\varepsilon \downarrow 0} \sup _{x>0}\left[F_{1}(x(1+2 \varepsilon))-F_{1}(x(1-2 \varepsilon))\right]=\underset{K \uparrow \infty}{\limsup } \limsup _{\varepsilon \downarrow 0} \sup _{0<x \leq K}\left[F_{1}(x(1+2 \varepsilon))-F_{1}(x(1-2 \varepsilon))\right]=0$,

since $F_{1}$ is uniformly continuous on $(0, K]$. This completes the proof of the first statement in Lemma 5.1 .

We turn to the second statement. The event that $k \in \partial \mathcal{T}_{m}^{(N)}$ implies that

$$
Y_{m}^{(i, N)} \geq \frac{1-\varepsilon^{2}}{\tau-1} \kappa^{-(k+1)} \log N
$$

By (5.6), we therefore conclude that when $\varepsilon$ is sufficiently small

$$
Y_{k}^{(i, N)} \geq \frac{1-\varepsilon}{\tau-1} \kappa^{-(k+1)} \log N
$$

which is equivalent to

$$
Z_{k}^{(i, N)} \geq N^{\frac{1-\varepsilon}{\kappa(\tau-1)}} \geq N^{\frac{1}{\kappa(\tau-1)}-\varepsilon}
$$

Therefore,

$$
\begin{aligned}
& \limsup _{\varepsilon \downarrow 0} \limsup _{m \rightarrow \infty} \limsup _{N \rightarrow \infty} \mathbb{P}\left(\left\{k \in \partial \mathcal{T}_{m}^{(i, N)}\right\} \cap\left\{\varepsilon \leq Y_{m}^{(i, N)} \leq \varepsilon^{-1}\right\} \cap\left\{Z_{k}^{(i, N)} \leq N^{\frac{1}{\kappa(\tau-1)}+\varepsilon}\right\}\right) \\
& \leq \limsup _{\varepsilon \downarrow 0} \limsup _{m \rightarrow \infty} \limsup _{N \rightarrow \infty} \mathbb{P}\left(\left\{k \in \mathcal{T}_{m}^{(i, N)}\right\} \cap\left\{\varepsilon \leq Y_{m}^{(i, N)} \leq \varepsilon^{-1}\right\} \cap\left\{Z_{k}^{(i, N)} \in\left[N^{\frac{1}{\kappa(\tau-1)}}{ }^{-\varepsilon}, N^{\frac{1}{\kappa(\tau-1)}+\varepsilon}\right]\right\}\right) \\
& =0 \text {, }
\end{aligned}
$$

which follows from the first statement in Lemma 5.1 with $u=\frac{1}{\kappa(\tau-1)}$.

Proof of Lemma 4.8, By (4.18), it suffices to prove that

$$
\underset{\varepsilon \downarrow 0}{\limsup } \limsup _{m \rightarrow \infty} \limsup _{N \rightarrow \infty} \mathbb{P}\left(\left\{H_{N}>k_{N}\right\} \cap E_{m, N} \cap F_{m, N} \cap\left\{\mathcal{B}_{N}\left(\varepsilon, k_{N}\right)=\varnothing\right\}\right)=0,
$$

which shows that in considering the event $\left\{H_{N}>k_{N}\right\} \cap E_{m, N} \cap F_{m, N}$, we may assume that $\mathcal{B}_{N}\left(\varepsilon, k_{N}\right) \neq \varnothing$.

We define the random variable $l^{*}$ by

$$
l^{*}=\sup \left\{k: \mathcal{B}_{N}(\varepsilon, k) \neq \varnothing\right\} .
$$

Observe that if $\mathcal{B}_{N}(\varepsilon, k)=\varnothing$, then $\mathcal{B}_{N}(\varepsilon, k+1)=\varnothing$, so that $l^{*}$ is well defined. Indeed, if $l \in$ $\mathcal{B}_{N}(\varepsilon, k+1)$ and $l \neq m$, then $l-1 \in \mathcal{B}_{N}(\varepsilon, k)$. If, on the other hand, $\mathcal{B}_{N}(\varepsilon, k+1)=\{m\}$, then also $m \in \mathcal{B}_{N}(\varepsilon, k)$. Since $\left\{\mathcal{B}_{N}\left(\varepsilon, k_{N}\right)=\varnothing\right\}=\left\{k_{N} \geq l^{*}+1\right\}$, we therefore have

$$
\left\{\mathcal{B}_{N}\left(\varepsilon, k_{N}\right)=\varnothing\right\}=\left\{l^{*}<k_{N}\right\}=\left\{l^{*} \leq k_{N}-2\right\} \dot{\cup}\left\{l^{*}=k_{N}-1\right\} .
$$


We deal with each of the two events separately. We start with the first.

Since the sets $\mathcal{B}_{N}(\varepsilon, k)$ are $Z_{m}$-measurable, we obtain, as in (4.30),

$$
\begin{aligned}
\mathbb{P}\left(\left\{H_{N}>k_{N}\right\} \cap E_{m, N} \cap F_{m, N} \cap\left\{l^{*} \leq k_{N}-2\right\}\right) & \leq \mathbb{P}\left(\left\{H_{N}>l^{*}+2\right\} \cap E_{m, N} \cap F_{m, N}\right) \\
& =\mathbb{E}\left[\mathbf{1}_{E_{m, N} \cap F_{m, N}} P_{m}\left(l^{*}+2, k_{1}\right)\right]+o_{N, m, \varepsilon}(1) .
\end{aligned}
$$

We then use (4.40) to bound

$$
\mathbb{E}\left[\mathbf{1}_{E_{m, N} \cap F_{m, N}} P_{m}\left(l^{*}+2, k_{1}\right)\right] \leq \mathbb{E}\left[\mathbf{1}_{E_{m, N} \cap F_{m, N}} \min _{k_{1} \in \mathcal{B}_{N}\left(\varepsilon, l^{*}\right)} \exp \left\{-\frac{Z_{k_{1}+1}^{(1, N)} Z_{l^{*}+2-k_{1}}^{(2, N)}}{2 L_{N}}\right\}\right] .
$$

Now, since $\mathcal{B}_{N}\left(\varepsilon, l^{*}\right) \neq \varnothing$, we can pick $k_{1}$ such that $k_{1}-1 \in \mathcal{B}_{N}\left(\varepsilon, l^{*}\right)$. Since $\mathcal{B}_{N}\left(\varepsilon, l^{*}+1\right)=\varnothing$, we have $k_{1}-1 \notin \mathcal{B}_{N}\left(\varepsilon, l^{*}+1\right)$, implying $l^{*}+1-k_{1} \in \mathcal{T}_{m}^{(2, N)}$ and $l^{*}+2-k_{1} \notin \mathcal{T}_{m}^{(2, N)}$ so that, by (3.9), $Z_{l^{*}+2-k_{1}}^{(2, N)} \geq N^{\frac{1-\varepsilon}{\tau-1}}$.

Similarly, since $k_{1} \notin \mathcal{B}_{N}\left(\varepsilon, l^{*}+1\right)$ we have that $k_{1} \in \mathcal{T}_{m}^{(1, N)}$ and $k_{1}+1 \notin \mathcal{T}_{m}^{(1, N)}$, so that, again by (3.9), $Z_{k_{1}+1}^{(1, N)} \geq N^{\frac{1-\varepsilon}{\tau-1}}$. Therefore, since $L_{N} \geq N$, whp,

$$
\frac{Z_{k_{1}+1}^{(1, N)} Z_{l^{*}+2-k_{1}}^{(2, N)}}{L_{N}} \geq N^{\frac{2(1-\varepsilon)}{\tau-1}-1}
$$

and the exponent of $N$ is strictly positive for $\tau \in(2,3)$ and $\varepsilon>0$ small enough. This bounds the contribution in (5.27) due to $\left\{l^{*} \leq k_{N}-2\right\}$.

We proceed with the contribution due to $\left\{l^{*}=k_{N}-1\right\}$. In this case, there exists a $k_{1}$ with $k_{1}-1 \in \mathcal{B}_{N}\left(\varepsilon, k_{N}-1\right)$ so that $k_{1} \in \mathcal{T}_{m}^{(1, N)}$ and $k_{N}-k_{1} \in \mathcal{T}_{m}^{(2, N)}$. On the other hand, $\mathcal{B}_{N}\left(\varepsilon, k_{N}\right)=\varnothing$, which together with $k_{1}-1 \in \mathcal{B}_{N}\left(\varepsilon, k_{N}-1\right)$ implies that $k_{N}-k_{1} \in \mathcal{T}_{m}^{(2, N)}$, and $k_{N}-k_{1}+1 \notin \mathcal{T}_{m}^{(2, N)}$. Similarly, we obtain that $k_{1} \in \mathcal{T}_{m}^{(1, N)}$ and $k_{1}+1 \notin \mathcal{T}_{m}^{(1, N)}$. Using Proposition 3.3. we conclude that $Z_{k_{1}+1}^{(1, N)} \geq N^{\frac{1-\varepsilon}{\tau-1}}$.

There are two possible cases that we will treat separately: (a) $Z_{k_{N}-k_{1}}^{(2, N)} \leq N^{\frac{\tau-2}{\tau-1}+\varepsilon}$; and (b) $Z_{k_{N}-k_{1}}^{(2, N)}>N^{\frac{\tau-2}{\tau-1}+\varepsilon}$. By (5.3) and the fact that $k_{N}-k_{1} \in \partial \mathcal{T}_{m}^{(2, N)}$, case (a) has small probability, so we need to investigate case (b) only.

In case (b), we can bound

$$
\begin{aligned}
& \mathbb{P}\left(\left\{H_{N}>k_{N}\right\} \cap E_{m, N} \cap F_{m, N} \cap\left\{l^{*}=k_{N}-1\right\} \cap\left\{Z_{k_{N}-k_{1}}^{(2, N)}>N^{\frac{\tau-2}{\tau-1}+\varepsilon}\right\}\right) \\
& \quad=\mathbb{E}\left[\mathbf{1}_{E_{m, N} \cap F_{m, N} \cap\left\{l^{*}=k_{N}-1\right\}} \mathbf{1}_{\left\{Z_{k_{N}-k_{1}}^{(2, N)}>N^{\frac{\tau-2}{\tau-1}+\varepsilon}\right\} \cap\left\{k_{1}-1 \in \mathcal{B}_{N}\left(\varepsilon, k_{N}-1\right)\right\}} P_{m}\left(k_{N}, k_{1}\right)\right]+o_{N, m, \varepsilon}(1),
\end{aligned}
$$

and again use (4.40) to obtain

$$
\begin{aligned}
& \mathbb{P}\left(\left\{H_{N}>k_{N}\right\} \cap E_{m, N} \cap F_{m, N} \cap\left\{l^{*}=k_{N}-1\right\} \cap\left\{Z_{k_{N}-k_{1}}^{(2, N)}>N^{\frac{\tau-2}{\tau-1}+\varepsilon}\right\}\right) \\
& \leq \mathbb{E}\left[\mathbf { 1 } _ { E _ { m , N } \cap F _ { m , N } ( \varepsilon ) \cap \{ l ^ { * } = k _ { N } - 1 \} } \mathbf { 1 } _ { \{ Z _ { k _ { N } - k _ { 1 } } ^ { ( 2 , N ) } > N ^ { \frac { \tau - 2 } { \tau - 1 } + \varepsilon } \} \cap \{ k _ { 1 } - 1 \in \mathcal { B } _ { N } ( \varepsilon , k _ { N } - 1 ) \} } \operatorname { e x p } \left\{-\frac{\left.\left.Z_{k_{1}+1}^{(1, N)} Z_{k_{N}-k_{1}}^{(2, N)}\right\}\right]+o_{N, m, \varepsilon}}{2 L_{N}}(1)\right.\right.
\end{aligned}
$$

We note that by Proposition 3.3 and similarly to (5.28),

$$
Z_{k_{1}+1}^{(1, N)} Z_{k_{N}-k_{1}}^{(2, N)} \geq N^{\frac{1-\varepsilon}{\tau-1}} N^{\frac{\tau-2}{\tau-1}+\varepsilon}=N^{1+\left(1-\frac{1}{\tau-1}\right) \varepsilon}
$$

and again the exponent is strictly larger than 1, so that, following the arguments in (5.26 5.30), we obtain that also the contribution due to case (b) is small. 
Proof of Lemma 4.11, Recall that $x=\kappa^{t} Y_{m,+}^{(1, N)}$ and $y=\kappa^{n-t} Y_{m,+}^{(2, N)}$, and that $x \geq y$. The event $\mathcal{E}_{m, N}$ in 4.70 is equal to

$$
\frac{1-\varepsilon}{\tau-1} \log N \leq x \leq \frac{1+\varepsilon}{\tau-1} \log N, \quad \text { and } \quad x+y \leq(1+\varepsilon) \log N .
$$

Therefore, by (4.65),

$$
y \geq \frac{x}{\kappa} \geq(1-\varepsilon) \frac{\tau-2}{\tau-1} \log N .
$$

Also, by the bound on $x+y$ in (5.32) and the lower bound on $x$ in (5.32),

$$
y \leq(1+\varepsilon) \log N-x \leq(1+\varepsilon) \log N-\frac{1-\varepsilon}{\tau-1} \log N=\left(1+\varepsilon \frac{\tau}{\tau-2}\right) \frac{\tau-2}{\tau-1} \log N .
$$

Therefore, by multiplying the bounds on $x$ and $y$, we obtain

$$
(1-\varepsilon)^{2} \frac{\tau-2}{(\tau-1)^{2}} \log ^{2} N \leq \kappa^{k_{N}+1} Y_{m,+}^{(1, N)} Y_{m,+}^{(2, N)} \leq\left(1+\varepsilon \frac{\tau}{\tau-2}\right)(1+\varepsilon) \frac{\tau-2}{(\tau-1)^{2}} \log ^{2} N,
$$

and thus

$$
\mathbb{P}\left(E_{m, N} \cap \mathcal{E}_{m, N} \cap\left\{H_{N}>k_{N}\right\}\right) \leq \mathbb{P}\left((1-\varepsilon)^{2} \leq \frac{\kappa^{k_{N}+1}}{c \log ^{2} N} Y_{m,+}^{(1, N)} Y_{m,+}^{(2, N)} \leq\left(1+\varepsilon \frac{\tau}{\tau-2}\right)(1+\varepsilon)\right),
$$

where we abbreviate $c=\frac{\tau-2}{(\tau-1)^{2}}$. We conclude that

$$
\limsup _{\varepsilon \downarrow 0} \limsup _{m \rightarrow \infty} \limsup _{N \rightarrow \infty} \mathbb{P}\left(E_{m, N} \cap \mathcal{E}_{m, N} \cap\left\{H_{N}>k_{N}\right\}\right)=0,
$$

analogously to the final part of the proof of Lemma 5.1.

Proof of Lemma 4.12. We recall that $M_{l}=\min _{t \in \mathbb{Z}}\left(\kappa^{t} Y^{(1)}+\kappa^{c_{l}-t} Y^{(2)}\right)$. We repeat the arguments leading to (4.88 4.91) to see that, as first $N \rightarrow \infty$ and then $m \rightarrow \infty$,

$$
\begin{aligned}
\mathbb{P}\left(\widetilde{F}_{N}^{c} \cap \widetilde{G}_{N}^{c} \cap E_{m, N}\right) & \leq \mathbb{P}\left(-\varepsilon \leq M_{l}-\kappa^{-a_{N}-\lceil l / 2\rceil} \leq \varepsilon, Y^{(1)} Y^{(2)}>0\right)+o_{N, m}(1) \\
& =q^{2} \mathbb{P}\left(-\varepsilon \leq M_{l}-\kappa^{-a_{N}-\lceil l / 2\rceil} \leq \varepsilon \mid Y^{(1)} Y^{(2)}>0\right)+o_{N, m}(1) .
\end{aligned}
$$

Recall from Section 2 that, conditionally on $Y^{(1)} Y^{(2)}>0$, the random variable $M_{l}$ has a density. Recall that we denoted the distribution function of $M_{l}$ given $Y^{(1)} Y^{(2)}>0$ by $F_{2}$. Furthermore, $\kappa^{-a_{N}-\lceil l / 2\rceil} \in I_{l}=\left[\kappa^{-\lceil l / 2\rceil}, \kappa^{-\lceil l / 2\rceil+1}\right]$, so that, uniformly in $N$,

$$
\mathbb{P}\left(-\varepsilon \leq M_{l}-\kappa^{-a_{N}-\lceil l / 2\rceil} \leq \varepsilon \mid Y^{(1)} Y^{(2)}>0\right) \leq \sup _{u \in I_{l}}\left[F_{2}(u+\varepsilon)-F_{2}(u-\varepsilon)\right]=0,
$$

where the conclusion follows by repeating the argument leading to (5.18). This completes the proof of Lemma 4.12

\section{A Appendix: Proof of Propositions 3.13 .3}

The appendix is organised as follows. In Section A.1 we prove three lemmas that are used in Section A.2 to prove Proposition 3.1. In Section A.3 we continue with preparations for the proofs of Proposition 3.2 and 3.3. In this section we formulate key Proposition A.3.2, which will be proved in Section A.4 In Section A.5 we end the appendix with the proofs of Proposition 3.2 and 3.3. 


\section{A.1 Some preparatory lemmas}

In order to prove Proposition 3.1 we make essential use of three lemmas, that also play a key role in Section A.4 below. The first of these three lemmas investigates the tail behaviour of $1-G(x)$ under Assumption [1.1. Recall that $G$ is the distribution function of the probability mass function $\left\{g_{j}\right\}$, defined in (1.8).

Lemma A.1.1 If $F$ satisfies Assumption 1.1 then there exists $K_{\tau}>0$ such that for $x$ large enough

$$
x^{2-\tau-K_{\tau} \gamma(x)} \leq 1-G(x) \leq x^{2-\tau+K_{\tau} \gamma(x)},
$$

where $\gamma(x)=(\log x)^{\gamma-1}, \gamma \in[0,1)$.

Proof. Using definition (1.8) we rewrite $1-G(x)$ as

$$
1-G(x)=\sum_{j=x+1}^{\infty} \frac{(j+1) f_{j+1}}{\mu}=\frac{1}{\mu}\left[(x+2)[1-F(x+1)]+\sum_{j=x+2}^{\infty}[1-F(j)]\right] .
$$

Then we use [20, Theorem 1, p. 281], together with the fact that $1-F(x)$ is regularly varying with exponent $1-\tau \neq 1$ to deduce that there exists a constant $c=c_{\tau}>0$ such that

$$
\sum_{j=x+2}^{\infty}[1-F(j)] \leq c_{\tau}(x+2)[1-F(x+2)] .
$$

Hence, if $F$ satisfies Assumption [1.1 then

$$
\begin{aligned}
1-G(x) & \geq \frac{1}{\mu}(x+2)[1-F(x+1)] \geq x^{2-\tau-K_{\tau} \gamma(x)}, \\
1-G(x) & \leq \frac{1}{\mu}(c+1)(x+2)[1-F(x+1)] \leq x^{2-\tau+K_{\tau} \gamma(x)},
\end{aligned}
$$

for some $K_{\tau}>0$ and large enough $x$.

Remark A.1.2 It follows from Assumption 1.1 and Lemma A.1.1 that for each $\varepsilon>0$ and sufficiently large $x$,

$$
\begin{aligned}
& x^{1-\tau-\varepsilon} \leq 1-F(x) \leq x^{1-\tau+\varepsilon}, \\
& x^{2-\tau-\varepsilon} \leq 1-G(x) \leq x^{2-\tau+\varepsilon} .
\end{aligned}
$$

We will often use (A.1.2) with $\varepsilon$ replaced by $\varepsilon^{6}$.

Let us define for $\varepsilon>0$,

$$
\alpha=\frac{1-\varepsilon^{5}}{\tau-1}, \quad h=\varepsilon^{6},
$$

and the auxiliary event $F_{\varepsilon}$ by

$$
F_{\varepsilon}=\left\{\forall 1 \leq x \leq N^{\alpha}:\left|G(x)-G^{(N)}(x)\right| \leq N^{-h}[1-G(x)]\right\},
$$

where $G^{(N)}$ is the (random) distribution function of $\left\{g_{j}^{(N)}\right\}$, defined in (3.2).

Lemma A.1.3 For $\varepsilon$ small enough, and $N$ sufficiently large,

$$
\mathbb{P}\left(F_{\varepsilon}^{c}\right) \leq N^{-h}
$$


Proof. First, we rewrite $1-G^{(N)}(x)$, for $x \in \mathbb{N}_{0}$, in the following way:

$$
\begin{aligned}
1-G^{(N)}(x) & =\sum_{n=x+1}^{\infty} g_{n}^{(N)}=\frac{1}{L_{N}} \sum_{j=1}^{N} \sum_{n=x+1}^{\infty} D_{j} \mathbf{1}_{\left\{D_{j}=n+1\right\}}=\frac{1}{L_{N}} \sum_{j=1}^{N} D_{j} \mathbf{1}_{\left\{D_{j} \geq x+2\right\}} \\
& =\frac{1}{L_{N}} \sum_{j=1}^{N} \sum_{l=1}^{D_{j}} \mathbf{1}_{\left\{D_{j} \geq x+2\right\}}=\frac{1}{L_{N}} \sum_{l=1}^{\infty} \sum_{j=1}^{N} \mathbf{1}_{\left\{D_{j} \geq(x+2) \vee l\right\}},
\end{aligned}
$$

where $x \vee l$ is the maximum of $x$ and $l$. Writing

$$
B_{y}^{(N)}=\sum_{j=1}^{N} \mathbf{1}_{\left\{D_{j} \geq y\right\}}
$$

we thus end up with

$$
1-G^{(N)}(x)=\frac{1}{L_{N}} \sum_{l=1}^{\infty} B_{(x+2) \vee l}^{(N)} .
$$

We have a similar expression for $1-G(x)$ that reads

$$
1-G(x)=\frac{1}{\mu} \sum_{l=1}^{\infty} \mathbb{P}\left(D_{1} \geq(x+2) \vee l\right) .
$$

Therefore, with

$$
\beta=\frac{1-h}{\tau-1}, \quad \text { and } \quad \chi=\frac{1+2 h}{\tau-1},
$$

we can write

$$
\begin{aligned}
{\left[G(x)-G^{(N)}(x)\right]=} & \left(\frac{N \mu}{L_{N}}-1\right)[1-G(x)] \\
& +\frac{1}{L_{N}} \sum_{l=1}^{N^{\beta}}\left[B_{(x+2) \vee l}^{(N)}-N \mathbb{P}\left(D_{1} \geq(x+2) \vee l\right)\right] \\
& +\frac{1}{L_{N}} \sum_{l=N^{\beta}+1}^{N \chi}\left[B_{(x+2) \vee l}^{(N)}-N \mathbb{P}\left(D_{1} \geq(x+2) \vee l\right)\right] \\
& +\frac{1}{L_{N}} \sum_{l=N \chi}^{\infty}\left[B_{(x+2) \vee l}^{(N)}-N \mathbb{P}\left(D_{1} \geq(x+2) \vee l\right)\right] .
\end{aligned}
$$

Hence, for large enough $N$ and $x \leq N^{\alpha}<N^{\beta}<N^{\chi}$, we can bound

$$
\begin{aligned}
R_{N}(x) \equiv\left|G(x)-G^{(N)}(x)\right| \leq & \left|\frac{N \mu}{L_{N}}-1\right|[1-G(x)] \\
& +\frac{1}{L_{N}} \sum_{l=1}^{N^{\beta}}\left|B_{(x+2) \vee l}^{(N)}-N \mathbb{P}\left(D_{1} \geq(x+2) \vee l\right)\right| \\
& +\frac{1}{L_{N}} \sum_{l=N^{\beta}+1}^{N^{\beta}}\left|B_{l}^{(N)}-N \mathbb{P}\left(D_{1} \geq l\right)\right| \\
& +\frac{1}{L_{N}} \sum_{l=N \chi+1}^{\infty} B_{l}^{(N)} \\
& +\frac{1}{L_{N}} \sum_{l=N \chi+1}^{\infty} N \mathbb{P}\left(D_{1} \geq l\right) .
\end{aligned}
$$

We use A.1.2(b)) to conclude that, in order to prove $\mathbb{P}\left(F_{\varepsilon}^{c}\right) \leq N^{-h}$, it suffices to show that

$$
\mathbb{P}\left(\bigcup_{1 \leq x \leq N^{\alpha}}\left\{\left|R_{N}(x)\right|>C_{g} N^{-h} x^{2-\tau-h}\right\}\right) \leq N^{-h}
$$

for large enough $N$, and for some $C_{g}$, depending on distribution function $G$. We will define an auxiliary event $A_{N, \varepsilon}$, such that $\left|R_{N}(x)\right|$ is more easy to bound on $A_{N, \varepsilon}$ and such that $\mathbb{P}\left(A_{N, \varepsilon}^{c}\right)$ is 
sufficiently small. Indeed, we define, with $A=3(\beta+2 h)$,

$$
\begin{aligned}
& A_{N, \varepsilon}(a)=\left\{\left|\frac{N \mu}{L_{N}}-1\right| \leq N^{-3 h}\right\}, \\
& A_{N, \varepsilon}(b)=\left\{\max _{1 \leq j \leq N} D_{j} \leq N^{\chi}\right\}, \\
& A_{N, \varepsilon}(c)=\bigcap_{1 \leq x \leq N^{\beta}}\left\{\left|B_{x}^{(N)}-N \mathbb{P}\left(D_{1} \geq x\right)\right| \leq \sqrt{A(\log N) N \mathbb{P}\left(D_{1} \geq x\right)}\right\},
\end{aligned}
$$

and

$$
A_{N, \varepsilon}=A_{N, \varepsilon}(a) \cap A_{N, \varepsilon}(b) \cap A_{N, \varepsilon}(c) .
$$

By intersecting with $A_{N, \varepsilon}$ and its complement, we have

$$
\begin{aligned}
\mathbb{P}\left(\bigcup_{1 \leq x \leq N^{\alpha}}\left\{\left|R_{N}(x)\right|>C_{g} N^{-h} x^{2-\tau-h}\right\}\right) \\
\leq \mathbb{P}\left(A_{N, \varepsilon} \cap\left\{\bigcup_{1 \leq x \leq N^{\alpha}}\left\{\left|R_{N}(x)\right|>C_{g} N^{-h} x^{2-\tau-h}\right\}\right\}\right)+\mathbb{P}\left(A_{N, \varepsilon}^{c}\right) .
\end{aligned}
$$

We will prove that $\mathbb{P}\left(A_{N, \varepsilon}^{c}\right) \leq N^{-h}$, and that on the event $A_{N, \varepsilon}$, and for each $1 \leq x \leq N^{\alpha}$, the right hand side of A.1.11 can be bounded by $C_{g} N^{-h} x^{2-\tau-h}$. We start with the latter statement.

Consider the right hand side of (A.1.11). Clearly, on $A_{N, \varepsilon}(a)$, the first term of $\left|R_{N}(x)\right|$ is bounded by $N^{-3 h}[1-G(x)] \leq C_{g} N^{-3 h} x^{2-\tau+h} \leq C_{g} N^{-h} x^{2-\tau-h}$, where the one but last inequality follows from (A.1.2(b)), and the last since $x \leq N^{\alpha}<N$ so that $x^{2 h}<N^{2 h}$. Since for $l>N^{\chi}$ and each $j, 1 \leq j \leq N$, we have $D_{j}>l$ is the empty set on $A_{N, \varepsilon}(b)$, the one but last term of $\left|R_{N}(x)\right|$ vanish on $A_{N, \varepsilon}(b)$. The last term of $\left|R_{N}(x)\right|$ can for $N$ large be bounded, using the inequality $L_{N} \geq N$ and A.1.2(a)),

$$
\frac{1}{L_{N}} \sum_{l=N \chi+1}^{\infty} N \mathbb{P}\left(D_{1} \geq l\right) \leq \sum_{l=N \chi+1}^{\infty} l^{1-\tau+h} \leq \frac{N^{\chi(2-\tau+h)}}{\tau-2}<C_{g} N^{-h+\alpha(2-\tau+h)} \leq C_{g} N^{-h} x^{2-\tau-h},
$$

for all $x \leq N^{\alpha}$, and where we also used that for $\varepsilon$ sufficiently small and $\tau>2$,

$$
\chi(2-\tau+h)<-h+\alpha(2-\tau+h) .
$$

We bound the third term of $\left|R_{N}(x)\right|$ as

$$
\begin{aligned}
\frac{1}{L_{N}} \sum_{l=N^{\beta}+1}^{N^{\chi}}\left|B_{l}^{(N)}-N \mathbb{P}\left(D_{1} \geq l\right)\right| & \leq \frac{1}{N} \sum_{l=N^{\beta}+1}^{N^{\chi}}\left[B_{l}^{(N)}+N \mathbb{P}\left(D_{1} \geq l\right)\right] \\
& \leq N^{\chi}\left[N^{-1} B_{N^{\beta}}^{(N)}+\mathbb{P}\left(D_{1} \geq N^{\beta}\right)\right] .
\end{aligned}
$$

We note that due to A.1.2(a)),

$$
\mathbb{P}\left(D_{1} \geq N^{\beta}\right) \geq N^{\beta(1-\tau-h)},
$$

for large enough $N$, so that

$$
a_{N}=\sqrt{A(\log N) N \mathbb{P}\left(D_{1} \geq N^{\beta}\right)} \leq N \mathbb{P}\left(D_{1} \geq N^{\beta}\right) .
$$

Therefore, on $A_{N, \varepsilon}(c)$, we obtain that

$$
B_{N^{\beta}}^{(N)} \leq 2 N \mathbb{P}\left(D_{1} \geq N^{\beta}\right),
$$

for $\varepsilon$ small enough and large enough $N$. Furthermore as $\varepsilon \downarrow 0$,

$$
N^{\chi+\beta(1-\tau+h)}<C_{g} N^{-h+\alpha(2-\tau-h)} \leq C_{g} N^{-h} x^{2-\tau-h},
$$


for $x \leq N^{\alpha}, 2-\tau-h<0$, because (after multiplying by $\tau-1$ and dividing by $\varepsilon^{5}$ )

$$
\chi+\beta(1-\tau+h)<-h+\alpha(2-\tau-h), \quad \text { or } \quad \varepsilon(2+2 \tau-h)<\tau-2+h,
$$

as $\varepsilon$ is sufficiently small. Thus, the third term of $\left|R_{N}(x)\right|$ satisfies the required bound.

We bound the second term of $\left|R_{N}(x)\right|$ on $A_{N, \varepsilon}(a) \cap A_{N, \varepsilon}(c)$ by

$$
\frac{1}{N} \sum_{l=1}^{N^{\beta}} \sqrt{A(\log N) N \mathbb{P}\left(D_{1} \geq(x+2) \vee l\right)}=\frac{\sqrt{A \log N}}{\sqrt{N}} \sum_{l=1}^{N^{\beta}} \sqrt{\mathbb{P}\left(D_{1} \geq(x+2) \vee l\right)} .
$$

Let $c$ be a constant such that $\left(\mathbb{P}\left(D_{1}>x\right)\right)^{\frac{1}{2}} \leq c x^{(1-\tau+h) / 2}$, then for all $1 \leq x \leq N^{\alpha}$,

$$
\begin{gathered}
\frac{1}{L_{N}} \sum_{l=1}^{N^{\beta}}\left|B_{(x+2) \vee l}^{(N)}-N \mathbb{P}\left(D_{1} \geq(x+2) \vee l\right)\right| \leq \frac{c \sqrt{A \log N}}{\sqrt{N}} \sum_{l=1}^{N^{\beta}}((x+2) \vee l)^{(1-\tau+h) / 2} \\
\leq \frac{c \sqrt{A \log N}}{\sqrt{N}}\left[x^{(3-\tau+h) / 2}+N^{\beta(3-\tau+h) / 2}\right] \leq \frac{2 c \sqrt{A \log N}}{\sqrt{N}} N^{\beta(3-\tau+h) / 2} \\
\leq N^{h-1 / 2} N^{\beta(3-\tau+h) / 2}<C_{g} N^{-h} N^{\alpha(2-\tau-h)} \leq C_{g} N^{-h} x^{2-\tau-h}
\end{gathered}
$$

because

$$
h-1 / 2+\beta(3-\tau+h) / 2<-h+\alpha(2-\tau-h), \quad \text { or } \quad h(5 \tau-4-h)<2 \varepsilon^{5}(\tau-2+h),
$$

for $\varepsilon$ small enough and $\tau \in(2,3)$. We have shown that for $1 \leq x \leq N^{\alpha}, N$ sufficiently large, and on the event $A_{N, \varepsilon}$,

$$
\left|R_{N}(x)\right| \leq C_{g} N^{-h} x^{2-\tau-h} .
$$

It remains to prove that $\mathbb{P}\left(A_{N, \varepsilon}^{c}\right) \leq N^{-h}$. We use that

$$
\mathbb{P}\left(A_{N, \varepsilon}^{c}\right) \leq \mathbb{P}\left(A_{N, \varepsilon}(a)^{c}\right)+\mathbb{P}\left(A_{N, \varepsilon}(b)^{c}\right)+\mathbb{P}\left(A_{N, \varepsilon}(c)^{c}\right),
$$

and we bound each of the three terms separately.

The bound

$$
\mathbb{P}\left(A_{N, \varepsilon}(a)^{c}\right)=\mathbb{P}\left(\left|\frac{1}{N} \sum_{j=1}^{N}\left(D_{j}-\mu\right)\right|>N^{-3 h} \cdot L_{N} / N\right) \leq \frac{1}{3} N^{-h},
$$

follows, since $N^{-\frac{1}{\tau-1}} \sum_{j=1}^{N}\left(D_{j}-\mu\right)$, converges to a stable law, for $2<\tau<3$.

The bound on $\mathbb{P}\left(A_{N, \varepsilon}(b)^{c}\right)$ is a trivial estimate using (A.1.2(a)). Indeed, for $N$ large,

$$
\mathbb{P}\left(A_{N, \varepsilon}(b)^{c}\right)=\mathbb{P}\left(\max _{1 \leq j \leq N} D_{j}>N^{\chi}\right) \leq N \mathbb{P}\left(D_{1} \geq N^{\chi}\right) \leq N^{\chi(1-\tau+h)+1} \leq \frac{1}{3} N^{-h}
$$

for small enough $\varepsilon$, because $\tau>2+h$. For the third term $\mathbb{P}\left(A_{N, \varepsilon}(c)^{c}\right)$, we will use a bound given by Janson [24], which states that for a binomial random variable $X$ with parameters $N$ and $p$, and all $t>0$,

$$
\mathbb{P}(|X-N p| \geq t) \leq 2 \exp \left\{-\frac{t^{2}}{2(N p+t / 3)}\right\} .
$$

We will apply A.1.25) with $t=a_{N}(x)=\sqrt{A(\log N) N \mathbb{P}\left(D_{1} \geq x\right)}$, and obtain that uniformly in $x \leq N^{\alpha}$,

$$
\begin{aligned}
& \mathbb{P}\left(\left|B_{x}^{(N)}-N \mathbb{P}\left(D_{1} \geq x\right)\right|>a_{N}(x)\right) \leq 2 \exp \left\{-\frac{a_{N}(x)^{2}}{2\left(N \mathbb{P}\left(D_{1} \geq x\right)+a_{N}(x) / 3\right)}\right\} \\
& \leq 2 \exp \left\{-\frac{A \log N}{2\left(1+\frac{1}{3} \sqrt{A \log N /\left(N \mathbb{P}\left(D_{1} \geq N^{\alpha}\right)\right.}\right)}\right\} \leq 2 N^{-A / 3}
\end{aligned}
$$


because

$$
\frac{\log N}{N \mathbb{P}\left(D_{1} \geq N^{\alpha}\right)} \leq \frac{\log N}{N^{1+\alpha(\tau-1-h)}} \rightarrow 0,
$$

as $N \rightarrow \infty$. Thus, A.1.26 gives us, using $A=3(\beta+2 h)$,

$$
\mathbb{P}\left(A_{N, \varepsilon}(c)^{c}\right) \leq \sum_{x=1}^{N^{\beta}} \mathbb{P}\left(\left|B_{x}^{(N)}-N \mathbb{P}\left(D_{1} \geq x\right)\right|>a_{N}(x)\right) \leq 2 N^{\beta-A / 3}=2 N^{-2 h} \leq \frac{1}{3} N^{-h} .
$$

This completes the proof of the lemma.

For the third lemma we introduce some further notation. For any $x \in \mathbb{N}$, define

$$
\hat{S}_{x}^{(N)}=\sum_{i=1}^{x} \hat{X}_{i}^{(N)}, \quad \hat{V}_{x}^{(N)}=\max _{1 \leq i \leq x} \hat{X}_{i}^{(N)},
$$

where $\left\{\hat{X}_{i}^{(N)}\right\}_{i=1}^{x}$ have the same law, say $\hat{H}^{(N)}$, but are not necessarily independent.

\section{Lemma A.1.4 (Sums with law $\hat{H}^{(N)}$ on the good event)}

(i) If $\hat{H}^{(N)}$ satisfies

$$
\left[1-\hat{H}^{(N)}(z)\right] \leq\left[1+2 N^{-h}\right][1-G(z)], \quad \forall z \leq y,
$$

then for all $x \geq 1$, there exists a constant $b^{\prime}$, such that:

$$
\mathbb{P}\left(\hat{S}_{x}^{(N)} \geq y\right) \leq b^{\prime} x\left[1+2 N^{-h}\right][1-G(y)]
$$

(ii) If $\hat{H}^{(N)}$ satisfies

$$
\left[1-\hat{H}^{(N)}(y)\right] \geq\left[1-2 N^{-h}\right][1-G(y)],
$$

and $\left\{\hat{X}_{i}^{(N)}\right\}_{i=1}^{x}$ are independent, then for all $x \geq 1$,

$$
\mathbb{P}\left(\hat{V}_{x}^{(N)} \leq y\right) \leq\left(1-\left[1-2 N^{-h}\right][1-G(y)]\right)^{x} .
$$

Proof. We first bound $\mathbb{P}\left(\hat{S}_{x}^{(N)} \geq y\right)$. We write

$$
\mathbb{P}\left(\hat{S}_{x}^{(N)} \geq y\right) \leq \mathbb{P}\left(\hat{S}_{x}^{(N)} \geq y, \hat{V}_{x}^{(N)} \leq y\right)+\mathbb{P}\left(\hat{V}_{x}^{(N)}>y\right) .
$$

The second term is bounded due to A.1.28 by

$$
x \mathbb{P}\left(\hat{X}_{1}^{(N)}>y\right)=x\left[1-\hat{H}^{(N)}(y)\right] \leq x\left[1+2 N^{-h}\right][1-G(y)] .
$$

We use the Markov inequality and (A.1.28) to bound the first term on the right-hand side of (A.1.32) by

$$
\begin{gathered}
\mathbb{P}\left(\hat{S}_{x}^{(N)} \geq y, \hat{V}_{x}^{(N)} \leq y\right) \leq \frac{1}{y} \mathbb{E}\left(\hat{S}_{x}^{(N)} \mathbf{1}_{\left\{\hat{V}_{x}^{(N)} \leq y\right\}}\right) \leq \frac{x}{y} \mathbb{E}\left(\hat{X}_{1}^{(N)} \mathbf{1}_{\left\{\hat{X}_{1}^{(N)} \leq y\right\}}\right) \\
\leq \frac{x}{y} \sum_{i=1}^{y}\left[1-\hat{H}^{(N)}(i)\right] \leq \frac{x}{y}\left[1+2 N^{-h}\right] \sum_{i=1}^{y}[1-G(i)] .
\end{gathered}
$$


For the latter sum, we use [20. Theorem 1(b), p. 281], together with the fact that $1-G(y)$ is regularly varying with exponent $2-\tau \neq 1$, to deduce that there exists a constant $c_{1}$ such that

$$
\sum_{i=1}^{y}[1-G(i)] \leq c_{1} y[1-G(y)] .
$$

Combining (A.1.32), A.1.33), A.1.34 and (A.1.35), we conclude that

$$
\left.\mathbb{P}\left(\hat{S}_{x}^{(N)} \geq y\right) \leq b^{\prime} x\left[1+2 N^{-h}\right][1-G(y))\right]
$$

where $b^{\prime}=c_{1}+1$. This completes the proof of Lemma A.1.4(i).

For the proof of (ii), we use independence of $\left\{\hat{X}_{i}^{(N)}\right\}_{i=1}^{x}$, and condition A.1.30, to conclude that

$$
\mathbb{P}\left(\hat{V}_{x}^{(N)} \leq y\right)=\left(\hat{H}^{(N)}(y)\right)^{x}=\left(1-\left[1-\hat{H}^{(N)}(y)\right]\right)^{x} \leq\left(1-\left[1-2 N^{-h}\right][1-G(y)]\right)^{x} .
$$

Hence, (A.1.31) holds.

Remark A.1.5 In the proofs in the appendix, we will only use that

(i) the event $F_{\varepsilon}$ holds whp;

(ii) that $L_{N}$ is concentrated around its mean;

(iii) that the maximal degree is bounded by $N^{\chi}$ for any $\chi>1 /(\tau-1)$, with whp.

Moreover, the proof of Proposition [3.1 relies on [21, Proposition A.3.1], and in its proof it was further used that

(iv) $p_{N} \leq N^{\alpha_{2}}$, whp, for any $\alpha_{2}>0$, where $p_{N}$ is the total variation distance between $g$ and $g^{(N)}$, i.e.,

$$
p_{N}=\frac{1}{2} \sum_{n}\left|g_{n}-g_{n}^{(N)}\right|
$$

Therefore, if instead of taking the degrees i.i.d. with distribution $F$, we would take the degrees in an exchangeable way such that the above restrictions hold, then the proof carries on verbatim. In particular, this implies that our results also hold for the usual configuration model, where the degrees are fixed, as long as the above restrictions are satisfied.

\section{A.2 Proof of Proposition 3.1}

The proof makes use of [21, Proposition A.3.1], which proves the statement in Proposition 3.1]under an additional condition.

In order to state this condition, let, for $i=1,2,\left\{\hat{Z}_{j}^{(i, N)}\right\}_{j \geq 1}$ be two independent copies of the delayed BP, where $\hat{Z}_{1}^{(i, N)}$ has law $\left\{f_{n}\right\}$ given in (1.1), and where the offspring of any individual in generation $j$ with $j>1$ has law $\left\{g_{n}^{(N)}\right\}$, where $g_{n}^{(N)}$ is defined in (3.1). Then, the conclusion of Proposition 3.1 follows from [21, Proposition A.3.1], for any $m$ such that, for any $\eta>0$, and $i=1,2$,

$$
\mathbb{P}\left(\sum_{j=1}^{m} \hat{Z}_{j}^{(i, N)} \geq N^{\eta}\right)=o(1) .
$$

By exchangeability it suffices to prove A.2.1 for $i=1$ only, we can therefore simplify notation and write further $\hat{Z}_{k}^{(N)}$ instead of $\hat{Z}_{k}^{(i, N)}$. We turn to the proof of (A.2.1). 
By Lemma A.1.3 and A.1.2(b)), respectively, for every $\eta>0$, there exists a $c_{\eta}>0$, such that whp for all $x \leq N^{\alpha}$,

$$
1-G^{(N)}(x) \leq\left[1+2 N^{-h}\right][1-G(x)] \leq c_{\eta} x^{2-\tau+\eta} .
$$

We call a generation $j \geq 1$ good, when

$$
\hat{Z}_{j}^{(N)} \leq\left(\hat{Z}_{j-1}^{(N)} \log N\right)^{\frac{1}{\tau-2-\eta}}
$$

and bad otherwise, where as always $\hat{Z}_{0}^{(N)}=1$. We further write

$$
H_{m}=\{\text { generations } 1, \ldots, m \text { are good }\} \text {. }
$$

We will prove that when $H_{m}$ holds, then $\sum_{j=1}^{m} \hat{Z}_{j}^{(N)} \leq N^{\eta}$. Indeed, when generations $1, \ldots, m$ are all good, then, for all $j \leq m$,

$$
\hat{Z}_{j}^{(N)} \leq(\log N)^{\sum_{i=1}^{j}(\tau-2-\eta)^{-i}} .
$$

Therefore,

$$
\sum_{j=1}^{m} \hat{Z}_{j}^{(N)} \leq m(\log N)^{\sum_{i=1}^{m}(\tau-2-\eta)^{-i}} \leq m(\log N)^{\frac{(\tau-2-\eta)-m-2}{(\tau-2-\eta)-1}-1} \leq N^{\eta},
$$

for any $\eta>0$, when $N$ is sufficiently large. We conclude that

$$
\mathbb{P}\left(\sum_{j=1}^{m} \hat{Z}_{j}^{(N)}>N^{\eta}\right) \leq \mathbb{P}\left(H_{m}^{c}\right)
$$

and Proposition 3.1 follows if we show that $\mathbb{P}\left(H_{m}^{c}\right)=o(1)$. In order to do so, we write

$$
\mathbb{P}\left(H_{m}^{c}\right)=\mathbb{P}\left(H_{1}^{c}\right)+\sum_{j=1}^{m-1} \mathbb{P}\left(H_{j+1}^{c} \cap H_{j}\right) .
$$

For the first term, we use $\mathrm{A.1.2}(\mathrm{a}))$ to deduce that

$$
\mathbb{P}\left(H_{1}^{c}\right)=\mathbb{P}\left(D_{1}>(\log N)^{\frac{1}{\tau-2-\eta}}\right) \leq(\log N)^{-\frac{\tau-1-\eta}{\tau-2-\eta}} \leq(\log N)^{-1} .
$$

For $1 \leq j \leq m$, we have $\hat{Z}_{j}^{(N)} \leq \sum_{k=1}^{m} \hat{Z}_{k}^{(N)}$, and using (A.2.6),

$$
\sum_{j=1}^{m} \hat{Z}_{j}^{(N)} \leq m(\log N)^{\frac{(\tau-2-\eta)-m-2}{(\tau-2-\eta)^{-1}-1}}=K_{N}
$$

Using Lemma A.1.4(i) with $\hat{H}^{(N)}=G^{(N)}, x=l$ and $y=v_{N}=(l \log N)^{\frac{1}{\tau-2-\eta}}$, where A.1.28 follows from (A.2.2), we obtain that

$$
\begin{gathered}
\mathbb{P}\left(H_{j+1}^{c} \cap H_{j}\right) \leq \sum_{l=1}^{K_{N}} \mathbb{P}\left(\hat{Z}_{j+1}^{(N)} \geq v_{N} \mid \hat{Z}_{j}^{(N)}=l\right) \mathbb{P}\left(\hat{Z}_{j}^{(N)}=l\right) \leq \max _{1 \leq l \leq K_{N}} \mathbb{P}\left(\hat{S}_{l}^{(N)} \geq v_{N}\right) \\
\leq \max _{1 \leq l \leq x_{o}} \mathbb{P}\left(\hat{S}_{l}^{(N)} \geq v_{N}\right)+b^{\prime} \max _{x_{o} \leq l \leq K_{N}} l\left[1+2 N^{-h}\right]\left[1-G\left(v_{N}\right)\right] .
\end{gathered}
$$

The case where $l \leq x_{o}$ can, by (A.2.2), be bounded as

$$
\begin{aligned}
& x_{o} \mathbb{P}\left(\sum_{1}^{l} \hat{X}_{j}^{(N)} \geq v_{N}\right) \leq x_{o} \mathbb{P}\left(\bigcup_{j=1}^{l}\left\{\hat{X}_{j}^{(N)} \geq v_{N} / l\right\}\right) \leq x_{o} \sum_{j=1}^{l} \mathbb{P}\left(\hat{X}_{j}^{(N)} \geq v_{N} / l\right) \\
& \quad \leq c_{\eta} l x_{o}\left(v_{N} / l\right)^{2-\tau+\eta}=\frac{c_{\eta} x_{o}}{l^{2-\tau+\eta}}(\log N)^{-1} \leq C(\log N)^{-1} .
\end{aligned}
$$

Furthermore by A.1.2(b)),

$$
\max _{x_{o} \leq l \leq K_{N}} l\left[1-G\left(v_{N}\right)\right] \leq \max _{x_{o} \leq l \leq K_{N}} l v_{N}^{1-\tau+\eta}<(\log N)^{-1} .
$$

This completes the proof of Proposition 3.1 


\section{A.3 Some further preparations}

Before we can prove Propositions 3.2 and 3.3 , we state a lemma that was proved in [21].

We introduce some notation. Suppose we have $L$ objects divided into $N$ groups of sizes $d_{1}, \ldots, d_{N}$, so that $L=\sum_{i=1}^{N} d_{i}$. Suppose we draw an object at random. This gives a distribution $g^{(\vec{d})}$, i.e.,

$$
g_{n}^{(\vec{d})}=\frac{1}{L} \sum_{i=1}^{N} d_{i} \mathbf{1}_{\left\{d_{i}=n+1\right\}}, \quad n=0,1, \ldots
$$

Clearly, $g^{(N)}=g^{(\vec{D})}$, where $\vec{D}=\left(D_{1}, \ldots, D_{N}\right)$. We further write

$$
G^{(\vec{d})}(x)=\sum_{n=0}^{x} g_{n}^{(\vec{d})} .
$$

We next label $M$ of the $L$ objects, and suppose that the distribution $G_{M}^{(\vec{d})}(x)$ is obtained in a similar way from drawing conditionally on drawing an unlabelled object. More precisely, we remove the labelled objects from all objects thus creating new $d_{1}^{\prime}, \ldots, d_{N}^{\prime}$, and we let $G_{M}^{(\vec{d})}(x)=G^{\left(\vec{d}^{\prime}\right)}(x)$. Even though this is not indicated, the law $G_{M}^{(\vec{d})}$ depends on what objects have been labelled.

Lemma A.3.1 below shows that the law $G_{M}^{(\vec{d})}$ can be stochastically bounded above and below by two specific ways of labeling objects. Before we can state the lemma, we need to describe those specific labellings.

For a vector $\vec{d}$, we denote by $d_{(1)} \leq d_{(2)} \leq \ldots \leq d_{(N)}$ the ordered coordinates. Then the laws $\bar{G}_{M}^{(\vec{d})}$ and $\underline{G}_{M}^{(\vec{d})}$, respectively, are defined by successively decreasing $d_{(N)}$ and $d_{(1)}$, respectively, by one. Thus,

$$
\begin{aligned}
& \bar{G}_{1}^{(\vec{d})}(x)=\frac{1}{L-1} \sum_{i=1}^{N-1} d_{(i)} \mathbf{1}_{\left\{d_{(i)} \leq x+1\right\}}+\frac{d_{(N)}-1}{L-1} \mathbf{1}_{\left\{d_{(N)}-1 \leq x+1\right\}}, \\
& \underline{G}_{1}^{(\vec{d})}(x)=\frac{1}{L-1} \sum_{i=2}^{N} d_{(i)} \mathbf{1}_{\left\{d_{(i)} \leq x+1\right\}}+\frac{d_{(1)}-1}{L-1} \mathbf{1}_{\left\{d_{(1)}-1 \leq x+1\right\}} .
\end{aligned}
$$

For $\bar{G}_{M}^{(\vec{d})}$ and $\underline{G}_{M}^{(\vec{d})}$, respectively, we perform the above change $M$ times, and after each repetition we reorder the groups. Here we note that when $d_{(N)}=1$ (in which case $d_{i}=1$, for all $i$ ), and for $\bar{G}_{1}^{(\vec{d})}$ we decrease $d_{(N)}$ by one, that we only keep the groups with $d_{i}=1$. The same rule applies when $d_{(1)}=1$ and for $\underline{G}_{1}^{(\vec{d})}$ we decrease $d_{(1)}$ by one. Thus, in these cases, the number of groups of objects, indicated by $N$, is decreased by 1 . Applying the above procedure to $\vec{d}=\left(D_{1}, \ldots, D_{N}\right)$ we obtain that, for all $x \geq 1$,

$$
\begin{aligned}
& \bar{G}_{M}^{(N)}(x) \equiv \bar{G}_{M}^{(\vec{D})}(x) \leq \frac{1}{L_{N}-M} \sum_{i=1}^{N} D_{i} \mathbf{1}_{\left\{D_{i} \leq x+1\right\}}=\frac{L_{N}}{L_{N}-M} G^{(N)}(x), \\
& \underline{G}_{M}^{(N)}(x) \equiv \underline{G}_{M}^{(\vec{D})}(x) \geq \frac{1}{L_{N}-M}\left[\sum_{i=1}^{N} D_{i} \mathbf{1}_{\left\{D_{i} \leq x+1\right\}}-M\right]=\frac{1}{L_{N}-M}\left[L_{N} G^{(N)}(x)-M\right],
\end{aligned}
$$

where equality is achieved precisely when $D_{(N)} \geq x+M$, and $\#\left\{i: D_{i}=1\right\} \geq M$, respectively.

Finally, for two distribution functions $F, G$, we write that $F \preceq G$ when $F(x) \geq G(x)$ for all $x$. Similarly, we write that $X \preceq Y$ when for the distribution functions $F_{X}, F_{Y}$ we have that $F_{X} \preceq F_{Y}$.

We next prove stochastic bounds on the distribution $G_{M}^{(\vec{d})}(x)$ that are uniform in the choice of the $M$ labelled objects. The proof of Lemma A.3.1 can be found in 21. 
Lemma A.3.1 For all choices of $M$ labelled objects

$$
\underline{G}_{M}^{(\vec{d})} \preceq G_{M}^{(\vec{d})} \preceq \bar{G}_{M}^{(\vec{d})} .
$$

Moreover, when $X_{1}, \ldots, X_{j}$ are draws from $G_{M_{1}}^{(\vec{d})}, \ldots, G_{M_{l}}^{(\overrightarrow{d)})}$, where the only dependence between the $X_{i}$ resides in the labelled objects, then

$$
\sum_{i=1}^{j} \underline{X}_{i} \preceq \sum_{i=1}^{j} X_{i} \preceq \sum_{i=1}^{j} \bar{X}_{i}
$$

where $\left\{\underline{X}_{i}\right\}_{i=1}^{j}$ and $\left\{\bar{X}_{i}\right\}_{i=1}^{j}$, respectively, are i.i.d. copies of $\underline{X}$ and $\bar{X}$ with laws $\underline{G}_{M}^{(N)}$ and $\bar{G}_{M}^{(N)}$ for $M=\max _{1 \leq i \leq l} M_{i}$, respectively.

We will apply Lemma A.3.1 to $G^{(\vec{D})}=G^{(N)}$.

\section{A.3.1 The inductive step}

Our key result, which will yield the proofs of Proposition 3.2 and 3.3. is Proposition A.3.2 below. This proposition will be proved in Section A.4 For its formulation we need some more notation.

As before we simplify notation and write further on $Z_{k}^{(N)}$ instead of $Z_{k}^{(i, N)}$. Similarly, we write $\mathcal{Z}_{k}$ instead of $\mathcal{Z}_{k}^{(i)}$ and $\mathcal{T}_{m}^{(N)}(\varepsilon)$ instead of $\mathcal{T}_{m}^{(i, N)}(\varepsilon)$. Recall that we have defined previously

$$
\kappa=\frac{1}{\tau-2}>1 \quad \text { and } \quad \alpha=\frac{1-\varepsilon^{5}}{\tau-1} .
$$

In the sequel we work with $Y_{k}^{(N)}>\varepsilon$, for $k$ large enough, i.e., we work with $Z_{k}^{(N)}>e^{\varepsilon \kappa^{k}}>1$, due to definition (3.3). Hence, we can treat these definitions as

$$
Y_{k}^{(N)}=\kappa^{-k} \log \left(Z_{k}^{(N)}\right) \quad \text { and } \quad Y_{k}=\kappa^{-k} \log \left(\mathcal{Z}_{k}\right) .
$$

With $\gamma$ defined in the Assumption 1.1, and $0<\varepsilon<3-\tau$, we take $m_{\varepsilon}$ sufficiently large to have

$$
\sum_{k=m_{\varepsilon}}^{\infty}(\tau-2+\varepsilon)^{k(1-\gamma)} \leq \varepsilon^{3} \quad \text { and } \quad \sum_{k=m_{\varepsilon}}^{\infty} k^{-2} \leq \varepsilon / 2 .
$$

For any $m_{\varepsilon} \leq m<k$, we denote

$$
M_{k}^{(N)}=\sum_{j=1}^{k} Z_{j}^{(N)}, \quad \text { and } \quad M_{k}=\sum_{j=1}^{k} \mathcal{Z}_{j} .
$$

As defined in Section 3 of [21] we speak of free stubs at level $l$, as the free stubs connected to nodes at distance $l-1$ from the root; the total number of free stubs, obtained immediately after pairing of all stubs of level $l-1$ equals $Z_{l}^{(N)}$. For any $l \geq 1$ and $1 \leq x \leq Z_{l-1}^{(N)}$, let $Z_{x, l}^{(N)}$ denote the number of constructed free stubs at level $l$ after pairing of the first $x$ stubs of $Z_{l-1}^{(N)}$. Note that for $x=Z_{l-1}^{(N)}$, we obtain $Z_{x, l}^{(N)}=Z_{l}^{(N)}$. For general $x$, the quantity $Z_{x, l}^{(N)}$ is loosely speaking the sum of the number of children of the first $x$ stubs at level $l-1$, and according to the coupling at fixed times (Proposition 3.1) this number is for fixed $l$, whp equal to the number of children of the first $x$ individuals in generation $l-1$ of the $\mathrm{BP}\left\{\mathcal{Z}_{k}\right\}_{k \geq 1}$.

We introduce the event $\hat{F}_{m, k}(\varepsilon)$,

$$
\begin{aligned}
& \left\{k \in \mathcal{T}_{m}^{(N)}(\varepsilon)\right\} \\
\hat{F}_{m, k}(\varepsilon)= & \cap\left\{\forall m<l \leq k-1:\left|Y_{l}^{(N)}-Y_{m}^{(N)}\right| \leq \varepsilon^{3}\right\} \\
& \cap\left\{\varepsilon \leq Y_{m}^{(N)} \leq \varepsilon^{-1}\right\} \\
& \cap\left\{M_{m}^{(N)} \leq 2 Z_{m}^{(N)}\right\} .
\end{aligned}
$$


In the proof of Proposition $A .3 .2$ we compare the quantity $Z_{x, l}^{(N)}$ to the sum $\sum_{i=1}^{x} X_{i, l-1}^{(N)}$ for part (a) and to $\max _{1 \leq i \leq x} X_{i, l-1}^{(N)}$ for part (b). We then couple $X_{i, l-1}^{(N)}$ to $\bar{X}_{i, l-1}^{(N)}$ for part (a) and to $\underline{X}_{i, l-1}^{(N)}$ for part (b). Among other things, the event $\hat{F}_{m, k}(\varepsilon)$ ensures that these couplings hold.

Proposition A.3.2 (Inductive step) Let $F$ satisfy Assumption 1.1. For $\varepsilon>0$ sufficiently small and $c_{\gamma}$ sufficiently large, there exist a constant $b=b(\tau, \varepsilon)>0$ such that, for $x=Z_{l-1}^{(N)} \wedge N^{\frac{(1-\varepsilon / 2)}{\kappa(\tau-1)}}$,

$$
\begin{aligned}
& \mathbb{P}\left(\hat{F}_{m, l}(\varepsilon) \cap\left\{Z_{x, l}^{(N)} \geq\left(l^{3} x\right)^{\kappa+c_{\gamma} \gamma(x)}\right\}\right) \leq b l^{-3}, \\
& \mathbb{P}\left(\hat{F}_{m, l}(\varepsilon) \cap\left\{Z_{x, l}^{(N)} \leq\left(\frac{x}{l^{3}}\right)^{\kappa-c_{\gamma} \gamma(x)}\right\}\right) \leq b l^{-3} .
\end{aligned}
$$

The proof of Proposition A.3.2 is quite technical and is given in Section A.4. In this section we give a short overview of the proof. For $l \geq 1$, let $S P G_{l}$ denote the shortest path graph containing all nodes on distance $l-1$, and including all stubs at level $l$, i.e., the moment we have $Z_{l}^{(N)}$ free stubs at level $l$. For $i \in\{1, \ldots, x\}$, let $X_{i, l-1}^{(N)}$ denote the number of brother stubs of a stub attached to $i^{\text {th }}$ stub of $S P G_{l-1}$ (see Figure 4).

a)

(1-2)-th level

(1-1)-th level

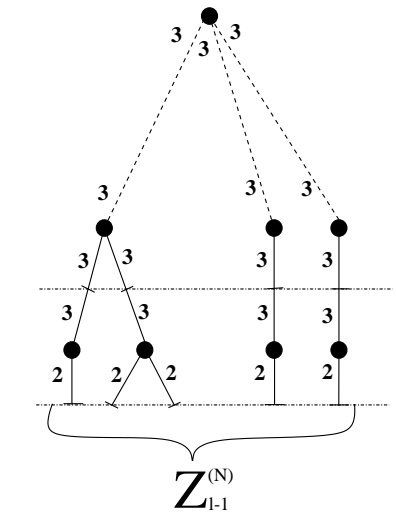

c)

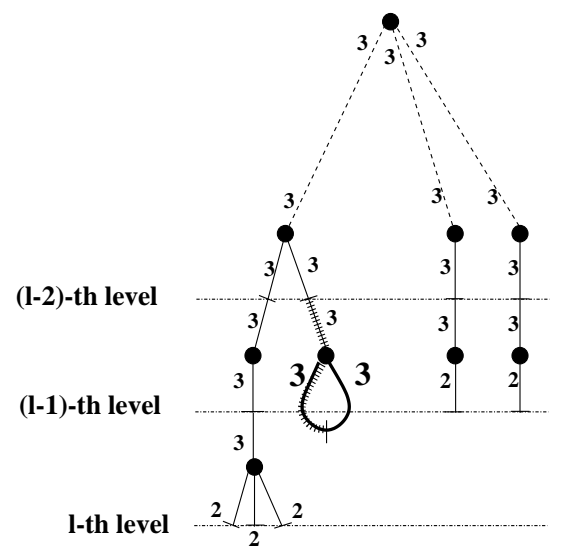

b)

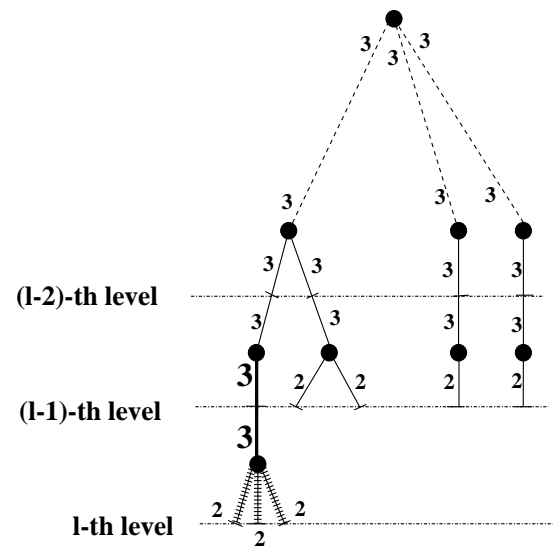

d)

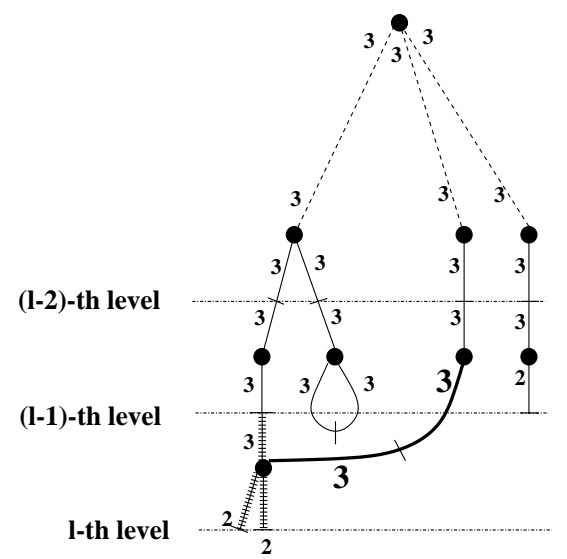

Figure 4: The building of the $l^{\text {th }}$ level of $S P G$. The last paired stubs are marked by thick lines, the brother stubs by dashed lines. In $a)$ the $(l-1)^{\text {st }}$ level is completed, in $\left.b\right)$ the pairing with a new node is described, in $\left.c\right)$ the pairing within the $(l-1)^{\text {st }}$ level is described, and in $\left.d\right)$ the pairing with already existing node at $l^{\text {th }}$ level is described. 
Because $Z_{x, l}^{(N)}$ is the number of free stubs at level $l$ after the pairing of the first $x$ stubs, one would expect that

$$
Z_{x, l}^{(N)} \sim \sum_{i=1}^{x} X_{i, l-1}^{(N)}
$$

where $\sim$ denotes that we have an uncontrolled error term. Indeed, the intuition behind (A.3.13) is that loops or cykels should be rare for small $l$. Furthermore, when $M_{l-1}^{(N)}$ is much smaller than $N$, then the law of $X_{i, l-1}^{(N)}$ should be quite close to the law $G^{(N)}$, which, in turn, by Lemma A.1.3 is close to $G$. If $X_{i, l-1}^{(N)}$ would have distribution $G(x)$, then we could use the theory of sums of random variables with infinite expectation, as well as extreme value theory, to obtain the inequalities of Proposition A.3.2

In order to make the above estimates rigorous, we use upper and lower bounds. We note that the right-hand side of (A.3.13) is a valid upper bound for $Z_{x, l}^{(N)}$. We show below that $X_{i, l-1}^{(N)}$ have the same law, and we wish to apply Lemma A.1.4(i). For this, we need to control the law $X_{i, l-1}^{(N)}$, for which we use Lemma A.3.1 to bound each $X_{i, l-1}^{(N)}$ from above by a random variable with law $\bar{G}_{M}^{(N)}$. This coupling makes sense only on the good event where $\bar{G}_{M}^{(N)}$ is sufficiently close to $G$.

For the lower bound, we have to do more work. The basic idea from the theory of sums of random variables with infinite mean is that the sum has the same order as the maximal summand. Therefore, we bound from below

$$
Z_{x, l}^{(N)} \geq \underline{Z}_{x, l}^{(N)}-x
$$

where

$$
\underline{Z}_{x, l}^{(N)}=\max _{1 \leq i \leq x} X_{i, l-1}^{(N)} .
$$

However, this lower bound is only valid when the chosen stub is not part of the shortest path graph up to that point. We show in Lemma A.3.4 below that the chosen stub has label 1 when $\underline{Z}_{x, l}^{(N)}>2 M_{l-1}^{(N)}$. In this case, A.3.14 follows since the $x-1$ remaining stubs can 'eat up' at most $x-1 \leq x$ stubs. To proceed with the lower bound, we bound $\left(X_{1, l-1}^{(N)}, \ldots, X_{x, l-1}^{(N)}\right)$ stochastically from below, using Lemma A.3.1 by an i.i.d. sequence of random variables with laws $\underline{G}_{M}^{(N)}$, where $M$ is chosen appropriately and serves as an upper bound on the number of stubs with label 3. Again on the good event, $\underline{G}_{M}^{(N)}$ is sufficiently close to $G$. Therefore, we are now faced with the problem of studying the maximum of a number of random variables with a law close to $G$. Here we can use Lemma A.1.4(ii), and we conclude in the proof of Proposition A.3.2 (a) that $\underline{Z}_{x, l}^{(N)}$ is to leading order equal to $x^{\kappa}$, when $x=Z_{l-1}^{(N)} \wedge N^{\frac{1-\varepsilon / 2}{\kappa(\tau-1)}}$. For this choice of $x$, we also see that $\underline{Z}_{x, l}^{(N)}$ is of bigger order than $M_{l-2}^{(N)}$, so that the basic assumption in the above heuristic is satisfied. This completes the overview of the proof.

We now state and prove the Lemmas A.3.3, A.3.4 The proof of Proposition A.3.2 then follows in Section A.4. We define the good event mentioned above by

$$
F_{\varepsilon, M}=\bigcap_{x=1}^{N^{\alpha}}\left\{\left[1-2 N^{-h}\right][1-G(x)] \leq 1-\bar{G}_{M}^{(N)}(x) \leq 1-\underline{G}_{M}^{(N)}(x) \leq\left[1+2 N^{-h}\right][1-G(x)]\right\} .
$$

The following lemma says that for $M \leq N^{\alpha}$ the probability of the good event is close to one.

Lemma A.3.3 Let F satisfy Assumption [1.1. Then, for $\varepsilon>0$ sufficiently small,

$$
\mathbb{P}\left(F_{\varepsilon, N^{\alpha}}^{c}\right) \leq N^{-h}, \quad \text { for large } N .
$$

Proof. Due to Lemma A.1.3 it suffices to show that for $\varepsilon$ small enough, and $N$ sufficiently we have

$$
F_{\varepsilon, N^{\alpha}}^{c} \subset F_{\varepsilon}^{c} .
$$


We will prove the equivalent statement that

$$
F_{\varepsilon} \subset F_{\varepsilon, N^{\alpha}}
$$

It follows from (A.3.5) and A.3.6 that for every $M$ and $x$

$$
1-\underline{G}_{M}^{(N)}(x) \leq 1-G^{(N)}(x) \leq 1-\bar{G}_{M}^{(N)}(x),
$$

and, in particular, that for $M \leq N^{\alpha}$,

$$
\left[1-\bar{G}_{M}^{(N)}(x)\right]-\left[1-\underline{G}_{M}^{(N)}(x)\right] \leq \frac{M}{L_{N}-M} \leq C N^{\alpha-1} .
$$

Then we use A.1.2(b)) to obtain that for all $x \leq N^{\alpha}, \varepsilon$ small enough, and $N$ sufficiently large,

$$
\begin{aligned}
& C N^{\alpha-1} \leq N^{\alpha-1+h}=N^{\frac{1-\varepsilon^{5}}{\tau-1}-1+\varepsilon^{6}}<N^{-2 \varepsilon^{6}} N^{\frac{1-\varepsilon^{5}}{\tau-1}\left(2-\tau-\varepsilon^{6}\right)} \\
& =N^{-2 h} N^{\alpha(2-\tau-h)} \leq N^{-2 h} x^{2-\tau-h} \leq N^{-h}[1-G(x)] .
\end{aligned}
$$

Therefore, for $M \leq N^{\alpha}$ and with the above choices of $\varepsilon, \alpha$ and $h$, we have on $F_{\varepsilon}$,

$$
\begin{aligned}
& {\left[1-\underline{G}_{M}^{(N)}(x)\right] \leq 1-G^{(N)}(x)+\left[1-\bar{G}_{M}^{(N)}(x)\right]-\left[1-\underline{G}_{M}^{(N)}(x)\right] \leq\left[1+2 N^{-h}\right][1-G(x)],} \\
& {\left[1-\underline{G}_{M}^{(N)}(x)\right] \geq 1-G^{(N)}(x)-\left[1-\bar{G}_{M}^{(N)}(x)\right]+\left[1-\underline{G}_{M}^{(N)}(x)\right] \geq\left[1-2 N^{-h}\right][1-G(x)],}
\end{aligned}
$$

i.e. we have (A.3.16), so that indeed $F_{\varepsilon} \subset F_{\varepsilon, N^{\alpha}}$.

For the coupling of $X_{i, l-1}^{(N)}$ with the random variables with laws $\underline{G}_{M}^{(N)}(x)$ and $\bar{G}_{M}^{(N)}(x)$ we need the following lemma.

Lemma A.3.4 For any $l \geq 1$ there are at most $2 M_{l}^{(N)}$ stubs with label 3 in $S P G_{l+1}$, while the number of stubs with label 2 is equal to $Z_{l+1}^{(N)}$.

Proof. The proof is by induction on $l$. There are $Z_{1}^{(N)}$ free stubs in $S P G_{1}$. Some of these stubs will be paired with stubs with label 2 or 3 , others will be paired to stubs with label 1 (see Figure 4). This gives us at most $2 Z_{1}^{(N)}$ stubs with label 3 in $S P G_{2}$. This initializes the induction. We next advance the induction. Suppose that for some $l \geq 1$ there are at most $2 M_{l}^{(N)}$ stubs with label 3 in $S P G_{l+1}$. There are $Z_{l+1}^{(N)}$ free stubs (with label 2) in $S P G_{l+1}$. Some of these stubs will be paired with stubs with label 2 or 3 , others will be linked with stubs with label 1 (again see Figure 4). This gives us at most $2 Z_{l+1}^{(N)}$ new stubs with label 3 in $S P G_{l+2}$. Hence the total number of these stubs is at most $2 M_{l}^{(N)}+2 Z_{l+1}^{(N)}=2 M_{l+1}^{(N)}$. This advances the induction hypothesis, and proves the claim.

\section{A.4 The proof of Proposition A.3.2}

We state and prove some consequences of the event $\hat{F}_{m, k}(\varepsilon)$, defined in (A.3.12). We refer to the remark, following definition (A.3.12), to explain where we use these consequences.

Lemma A.4.1 The event $\hat{F}_{m, k}(\varepsilon)$ implies, for sufficiently large $N$, the following bounds:

(a) $\quad M_{k-1}^{(N)}<N^{\frac{1-3 \varepsilon^{4} / 4}{\kappa(\tau-1)}}$

(b) for any $\delta>0, N^{-\delta} \leq k^{-3}$,

(c) $\quad \kappa^{k-1}\left(\varepsilon-\varepsilon^{3}\right) \leq \log \left(Z_{k-1}^{(N)}\right) \leq \kappa^{k-1}\left(\varepsilon^{-1}+\varepsilon^{3}\right)$, for $k-1 \geq m$,

$$
M_{k-1}^{(N)} \leq 2 Z_{k-1}^{(N)} \quad \text { for } \quad k-1 \geq m \text {. }
$$


Proof. Assume that A.3.12(a)-(d)) holds. We start by showing A.4.1(b)), which is evident if we show the following claim:

$$
k \leq \frac{\log \left(\frac{1-\varepsilon^{2}}{\varepsilon(\tau-1)} \log N\right)}{\log \kappa},
$$

for $N$ large enough. In order to prove (A.4.2), we note that if $k \in \mathcal{T}_{m}^{(N)}(\varepsilon)$ then, due to definition (3.5),

$$
\kappa^{k-m} \leq \frac{1-\varepsilon^{2}}{\tau-1} \frac{\log N}{\log \left(Z_{m}^{(N)}\right)}<\frac{1-\varepsilon^{2}}{\varepsilon(\tau-1)} \kappa^{-m} \log N,
$$

where the latter inequality follows from $Y_{m}^{(N)}>\varepsilon$ and (A.3.9). Multiplying by $\kappa^{m}$ and taking logarithms on both sides yields (A.4.2).

We now turn to A.4.1(a)). Since

$$
M_{k-1}^{(N)}=\sum_{l=1}^{k-1} Z_{l}^{(N)} \leq k \max _{1 \leq l \leq k-1} Z_{l}^{(N)},
$$

the inequality A.4.1(a)) follows when we show that for any $l \leq k-1$,

$$
Z_{l}^{(N)} \leq N^{\frac{1-\varepsilon^{4}}{\kappa(\tau-1)}}
$$

Observe that for $l<m$ we have, due to $\underline{A .3 .12}(\mathrm{c}))$ and $(\mathrm{A.3.12}(\mathrm{d}))$, for any $\varepsilon>0$ and $m$ fixed and by taking $N$ sufficiently large,

$$
Z_{l}^{(N)} \leq M_{m}^{(N)} \leq 2 Z_{m}^{(N)} \leq 2 e^{\kappa^{m} \varepsilon^{-1}}<N^{\frac{1-\varepsilon^{4}}{\kappa(\tau-1)}} .
$$

Consider $m \leq l \leq k-1$. Due to A.3.9), inequality A.4.4 is equivalent to

$$
\kappa^{l+1} Y_{l}^{(N)} \leq \frac{1-\varepsilon^{4}}{\tau-1} \log N
$$

To obtain A.4.6 we will need two inequalities. Firstly, A.3.12(a)) and $l+1 \leq k$ imply that

$$
\kappa^{l+1} Y_{m}^{(N)} \leq \frac{1-\varepsilon^{2}}{\tau-1} \log N .
$$

Secondly, (A.4.7) and (A.3.12(c)) imply that

$$
\kappa^{l+1} \leq \frac{1-\varepsilon^{2}}{\varepsilon(\tau-1)} \log N .
$$

Given (A.4.7) and A.3.12(b)), we obtain, when $Y_{m}^{(N)} \geq \varepsilon$, and for $m \leq l \leq k-1$,

$$
\begin{aligned}
\kappa^{l+1} Y_{l}^{(N)} & \leq \kappa^{l+1}\left(Y_{m}^{(N)}+\varepsilon^{3}\right) \leq \kappa^{l+1} Y_{m}^{(N)}\left(1+\varepsilon^{2}\right) \\
& \leq \frac{\left(1-\varepsilon^{2}\right)\left(1+\varepsilon^{2}\right)}{\tau-1} \log N=\frac{1-\varepsilon^{4}}{\tau-1} \log N .
\end{aligned}
$$

Hence we have (A.4.6) or equivalently (A.4.4) for $m \leq l \leq k-1$.

The bound in A.4.1(c)) is an immediate consequence of (A.3.9) and (A.3.12(b,c)) that imply for $k-1>m$,

$$
\varepsilon-\varepsilon^{3} \leq Y_{k-1}^{(N)} \leq \varepsilon^{-1}+\varepsilon^{3} .
$$

We complete the proof by establishing A.4.1(d)). We use induction to prove that for all $l \geq m$, the bound $M_{l}^{(N)} \leq 2 Z_{l}^{(N)}$ holds. The initialization of the induction hypothesis for $l=m$ follows from A.3.12(d)). So assume that for some $m \leq l<k-1$ the inequality $M_{l}^{(N)} \leq 2 Z_{l}^{(N)}$ holds, then

$$
M_{l+1}^{(N)}=Z_{l+1}^{(N)}+M_{l}^{(N)} \leq Z_{l+1}^{(N)}+2 Z_{l}^{(N)},
$$


so that it suffices to bound $2 Z_{l}^{(N)}$ by $Z_{l+1}^{(N)}$. We note that $\hat{F}_{m, k}(\varepsilon)$ implies that

$$
\left|Y_{l+1}^{(N)}-Y_{l}^{(N)}\right| \leq\left|Y_{l+1}^{(N)}-Y_{m}^{(N)}\right|+\left|Y_{l}^{(N)}-Y_{m}^{(N)}\right| \leq 2 \varepsilon^{3} \leq 3 \varepsilon^{2} Y_{l+1}^{(N)} .
$$

Therefore,

$$
2 Z_{l}^{(N)}=2 e^{\kappa^{l} Y_{l}^{(N)}} \leq 2 e^{\left(1+3 \varepsilon^{2}\right) \kappa^{l} Y_{l+1}^{(N)}}=2\left(Z_{l+1}^{(N)}\right)^{\left(1+3 \varepsilon^{2}\right) \kappa^{-1}} \leq Z_{l+1}^{(N)},
$$

when $\varepsilon>0$ is so small that $\omega=\left(1+3 \varepsilon^{2}\right) \kappa^{-1}<1$ and where we take $m$ large enough to ensure that for $l \geq m$, the lower bound $Z_{l+1}^{(N)}=\exp \left\{\kappa^{l+1} Y_{l+1}^{(N)}\right\}>\exp \left\{\kappa^{l+1} \varepsilon\right\}>2^{\frac{1}{1-\omega}}$ is satified.

Proof of Proposition $\underline{\mathbf{A . 3 . 2}}(\mathbf{a})$. Recall that $\alpha=\frac{1-\varepsilon^{5}}{\tau-1}$. We write

$$
\begin{aligned}
\mathbb{P}\left(\hat{F}_{m, l}(\varepsilon) \cap\left\{Z_{x, l} \geq\left(l^{3} x\right)^{\kappa+c_{\gamma} \gamma(x)}\right\}\right) & \leq \mathbb{P}_{N^{\alpha}}\left(\hat{F}_{m, l}(\varepsilon) \cap\left\{Z_{x, l} \geq\left(l^{3} x\right)^{\kappa+c_{\gamma} \gamma(x)}\right\}\right)+\mathbb{P}\left(F_{\varepsilon, N^{\alpha}}^{c}\right) \\
& \leq \mathbb{P}_{N^{\alpha}}\left(\hat{F}_{m, l}(\varepsilon) \cap\left\{Z_{x, l} \geq\left(l^{3} x\right)^{\kappa+c_{\gamma} \gamma(x)}\right\}\right)+l^{-3}
\end{aligned}
$$

where $\mathbb{P}_{M}$ is the conditional probability given that $F_{\varepsilon, M}$ holds, and where we have used Lemma A.3.3 with $N^{-h}<l^{-3}$. It remains to bound the first term on the right-hand side of (A.4.13). For this bound we aim to use Lemma A.1.4 Clearly we have

$$
Z_{x, l}^{(N)} \leq \sum_{i=1}^{x} X_{i, l-1}^{(N)},
$$

because loops and cycles can occur (in Figure 4 only the case $b$ ) contributes to $Z_{x, l}^{(N)}$, the cases $c$ ) and $d$ ) do not contribute). Since the free stubs of $S P G_{l-1}$ are exchangeable, each free stub will choose any stub with label unequal to 3 with the same probability. Therefore, all $X_{i, l-1}^{(N)}$ have the same law which we denote by $H^{(N)}$. Then we observe that due to (A.3.8), $X_{i, l-1}^{(N)}$ can be coupled with $\bar{X}_{i, l-1}^{(N)}$ having law $\bar{G}_{M}^{(N)}$, where $M$ is equal to the number of stubs with label 3 at the moment we generate $X_{i, l-1}^{(N)}$, which is at most the number of stubs with label 3 in $S P G_{l}$ plus 1 . The last number is due to Lemma A.3.4 at most $2 M_{l-1}^{(N)}+1$. By Lemma A.4.1 (a), we have that

$$
2 M_{l-1}^{(N)}+1 \leq 2 N^{\frac{1-3 \varepsilon^{4} / 4}{\kappa(\tau-1)}}+1 \leq N^{\frac{1-\varepsilon^{5}}{\tau-1}}=N^{\alpha},
$$

and hence, due to (A.3.8), we can take as the largest possible number $M=N^{\alpha}$. We now verify whether we can apply Lemma A.1.4(i). Observe that $x \leq N^{\frac{1-\varepsilon / 2}{\kappa(\tau-1)}}$ so that for $\mathrm{N}$ large and each $c_{\gamma}$, we have

$$
y=\left(l^{3} x\right)^{\kappa+c_{\gamma} \gamma(x)}<N^{\alpha},
$$

since by (A.4.2), we can bound $l$ by a double logarithm. Hence (A.1.28) holds, because we condition on $F_{\varepsilon, N^{\alpha}}$. We therefore can apply Lemma A.1.4 (i), with $\hat{S}_{x}^{(N)}=\sum_{i=1}^{x} \bar{X}_{i, l-1}^{(N)}, \hat{H}^{(N)}=\bar{G}_{N^{\alpha}}^{(N)}$, and obtain also using the upper bound in (A.1.1),

$$
\begin{gathered}
\mathbb{P}_{N^{\alpha}}\left(\hat{F}_{m, l}(\varepsilon) \cap\left\{Z_{x, l} \geq\left(l^{3} x\right)^{\kappa+c_{\gamma} \gamma(x)}\right\}\right) \leq b^{\prime} x\left[1+2 N^{-h}\right][1-G(y)] \\
\leq 2 b^{\prime} x y^{-\kappa^{-1}+K_{\tau} \gamma(y)}=2 b^{\prime} x\left(l^{3} x\right)^{\left(-\kappa^{-1}+K_{\tau} \gamma(y)\right)\left(\kappa+c_{\gamma} \gamma(x)\right)} \leq b l^{-3}
\end{gathered}
$$

if we show that

$$
c_{\gamma} \gamma(x)\left(-\kappa^{-1}+K_{\tau} \gamma(y)\right)+\kappa K_{\tau} \gamma(y)<0 .
$$

Inequality A.4.18 holds, because $\gamma(y)=(\log y)^{\gamma-1}, \gamma \in[0,1)$, can be made arbitrarily small by taking $y$ large, which follows from (A.4.1(c)): $l^{3} x \geq l^{3} \exp \left\{\kappa^{m} \varepsilon / 2\right\}$, and because $m$ can be taken large. 
Proof of Proposition A.3.2(b). Similarly to A.4.13), we have

$$
\mathbb{P}\left(\hat{F}_{m, l}(\varepsilon) \cap\left\{Z_{x, l}^{(N)} \leq\left(\frac{x}{l^{3}}\right)^{\kappa-c_{\gamma} \gamma(x)}\right\}\right) \leq \mathbb{P}_{N^{\alpha}}\left(\hat{F}_{m, l}(\varepsilon) \cap\left\{Z_{x, l}^{(N)} \leq\left(\frac{x}{l^{3}}\right)^{\kappa-c_{\gamma} \gamma(x)}\right\}\right)+l^{-3},
$$

and it remains to bound the first term on the right-hand side of (A.4.19). Recall that

$$
\underline{Z}_{x, l}^{(N)}=\max _{1 \leq i \leq x} X_{i, l-1}^{(N)},
$$

where, for $1 \leq i \leq x, X_{i, l-1}^{(N)}$ is the number of brother stubs of a stub attached to the $i^{\text {th }}$ free stub of $S P G_{l-1}$. Suppose we can bound the first term on the right-hand side of (A.4.19) by $b l^{-3}$, when $Z_{x, l}^{(N)}$ is replaced by $\underline{Z}_{x, l}^{(N)}$ after adding an extra factor 2 , e.g., suppose that

$$
\mathbb{P}_{N^{\alpha}}\left(\hat{F}_{m, l}(\varepsilon) \cap\left\{\underline{Z}_{x, l}^{(N)} \leq 2\left(\frac{x}{l^{3}}\right)^{\kappa-c_{\gamma} \gamma(x)}\right\}\right) \leq b l^{-3} .
$$

Then we bound

$$
\begin{aligned}
& \mathbb{P}_{N^{\alpha}}\left(\hat{F}_{m, l}(\varepsilon) \cap\left\{Z_{x, l}^{(N)} \leq\left(\frac{x}{l^{3}}\right)^{\kappa-c_{\gamma} \gamma(x)}\right\}\right) \\
& \leq \mathbb{P}_{N^{\alpha}}\left(\hat{F}_{m, l}(\varepsilon) \cap\left\{\underline{Z}_{x, l}^{(N)} \leq 2\left(\frac{x}{l^{3}}\right)^{\kappa-c_{\gamma} \gamma(x)}\right\}\right) \\
&+\mathbb{P}_{N^{\alpha}}\left(\hat{F}_{m, l}(\varepsilon) \cap\left\{Z_{x, l}^{(N)} \leq\left(\frac{x}{l^{3}}\right)^{\kappa-c_{\gamma} \gamma(x)}\right\} \cap\left\{\underline{Z}_{x, l}^{(N)}>2\left(\frac{x}{l^{3}}\right)^{\kappa-c_{\gamma} \gamma(x)}\right\}\right) .
\end{aligned}
$$

By assumption, the first term is bounded by $b l^{-3}$, and we must bound the second term. We will prove that the second term in (A.4.20) is equal to 0 .

For $x$ sufficiently large we obtain from $l \leq C \log x, \kappa>1$, and $\gamma(x) \rightarrow 0$,

$$
2\left(\frac{x}{l^{3}}\right)^{\kappa-c_{\gamma} \gamma(x)}>6 x
$$

Hence for $x=Z_{l-1}^{(N)}>\left(\varepsilon-\varepsilon^{3}\right) \kappa^{l-1}$, it follows from Lemma A.4.1 (d), that $\underline{Z}_{x, l}^{(N)}>2\left(\frac{x}{l^{3}}\right)^{\kappa-c_{\gamma} \gamma(x)}$ induces

$$
\underline{Z}_{x, l}^{(N)}>6 Z_{l-1}^{(N)} \geq 2 M_{l-1}^{(N)}+2 Z_{l-1}^{(N)} .
$$

On the other hand, when $x=N^{\frac{(1-\varepsilon / 2)}{\kappa(\tau-1)}}<Z_{l-1}^{(N)}$, then, by Lemma A.4.1(a), and where we use again $l \leq C \log x, \kappa>1$, and $\gamma(x) \rightarrow 0$,

$$
\begin{aligned}
\underline{Z}_{x, l}^{(N)} & \geq 2\left(\frac{x}{l^{3}}\right)^{\kappa-c_{\gamma} \gamma(x)}=2\left(\frac{N^{\frac{1-\varepsilon / 2}{\kappa(\tau-1)}}}{l^{3}}\right)^{\kappa-c_{\gamma} \gamma(x)} \\
& >2 N^{\frac{1-3 \varepsilon^{4} / 4}{\kappa(\tau-1)}}+2 N^{\frac{1-\varepsilon / 2}{\kappa(\tau-1)}}>2 M_{l-1}^{(N)}+2 x .
\end{aligned}
$$

We conclude that in both cases we have that $\underline{Z}_{x, l}^{(N)} \geq 2 M_{l-1}^{(N)}+2 x \geq 2 M_{l-2}^{(N)}+2 x$. We claim that the event $\underline{Z}_{x, l}^{(N)}>2 M_{l-2}^{(N)}+2 x$ implies that

$$
Z_{x, l}^{(N)} \geq \underline{Z}_{x, l}^{(N)}-x
$$

Indeed, let $i_{0} \in\{1, \ldots, N\}$ be the node such that

$$
D_{i_{0}}=\underline{Z}_{x, l}^{(N)}+1
$$

and suppose that $i_{0} \in S P G_{l-1}$. Then $D_{i_{0}}$ is at most the total number of stubs with labels 2 and 3, i.e., at most $2 M_{l-2}^{(N)}+2 x$. Hence $\underline{Z}_{x, l}^{(N)}<D_{i_{0}} \leq 2 M_{l-2}^{(N)}+2 x$, and this is a contradiction 
with the assumption that $\underline{Z}_{x, l}^{(N)}>2 M_{l-2}^{(N)}+2 x$. Since by definition $i_{0} \in S P G_{l}$, we conclude that $i_{0} \in S P G_{l} \backslash S P G_{l-1}$, which is equivalent to saying that the chosen stub with $\underline{Z}_{x, l}^{(N)}$ brother stubs had label 1. Then, on $\underline{Z}_{x, l}^{(N)}>2 M_{l-2}^{(N)}+2 x$, we have A.4.24). Indeed, the one stub from level $l-1$ connected to $i_{0}$ gives us $\underline{Z}_{x, l}^{(N)}$ free stubs at level $l$ and the other $x-1$ stubs from level $l-1$ can 'eat up' at most $x$ stubs.

We conclude from the above that

$$
\begin{aligned}
& \mathbb{P}_{N^{\alpha}}\left(\hat{F}_{m, l}(\varepsilon) \cap\left\{Z_{x, l}^{(N)} \leq\left(\frac{x}{l^{3}}\right)^{\kappa-c_{\gamma} \gamma(x)}\right\} \cap\left\{\underline{Z}_{x, l}^{(N)}>2\left(\frac{x}{l^{3}}\right)^{\kappa-c_{\gamma} \gamma(x)}\right\}\right) \\
& \quad \leq \mathbb{P}_{N^{\alpha}}\left(\hat{F}_{m, l}(\varepsilon) \cap\left\{Z_{x, l}^{(N)} \leq\left(\frac{x}{l^{3}}\right)^{\kappa-c_{\gamma} \gamma(x)}\right\} \cap\left\{Z_{x, l}^{(N)}>2\left(\frac{x}{l^{3}}\right)^{\kappa-c_{\gamma} \gamma(x)}-x\right\}\right)=0,
\end{aligned}
$$

since A.4.21) implies that

$$
2\left(\frac{x}{l^{3}}\right)^{\kappa-c_{\gamma} \gamma(x)}-x \geq\left(\frac{x}{l^{3}}\right)^{\kappa-c_{\gamma} \gamma(x)} .
$$

Below we prove in two steps that there exists $b$ such that

$$
\mathbb{P}_{N^{\alpha}}\left(\hat{F}_{m, l}(\varepsilon) \cap\left\{\underline{Z}_{x, l}^{(N)} \leq 2\left(\frac{x}{l^{3}}\right)^{\kappa-c_{\gamma} \gamma(x)}\right\}\right) \leq b l^{-3} .
$$

First we couple $\left\{X_{i, l-1}^{(N)}\right\}_{i=1}^{x}$ with a sequence of i.i.d. random variables $\left\{\underline{X}_{i, l-1}^{(N)}\right\}_{i=1}^{x}$ with law $\underline{G}_{N^{\alpha}}^{(N)}$, such that

$$
X_{i, l-1}^{(N)} \geq \underline{X}_{i, l-1}^{(N)}, \quad i=1,2, \ldots, x,
$$

and hence

$$
\underline{Z}_{x, l}^{(N)} \geq V_{x}^{(N)} \stackrel{\text { def }}{=} \max _{1 \leq i \leq x} \underline{X}_{i, l-1}^{(N)} .
$$

Then we apply Lemma A.1.4(ii) with $\hat{X}_{i}^{(N)}=\underline{X}_{i, l-1}^{(N)}$ and $y=2\left(x / l^{3}\right)^{\kappa-c_{\gamma} \gamma(x)}$.

We prove the fact that we can couple $\left\{X_{i, l-1}^{(N)}\right\}_{i=1}^{x}$ with a sequence of i.i.d. random variables $\left\{\underline{X}_{i, l-1}^{(N)}\right\}_{i=1}^{x}$ with law $\underline{G}_{N^{\alpha}}^{(N)}$ by induction on $x$. For $x=1$, the claim follows from Lemma A.3.1 with $l=1$. Observe that for the $x^{\text {th }}$ stub at level $l-1$, we sample a uniform stub from the stubs with labels 1 and 2. Hence, due to A.3.8), and conditionally on $\left\{X_{i, l-1}^{(N)}\right\}_{i=1}^{x-1}$, we can bound $X_{x, l-1}^{(N)}$ from below by $\underline{X}_{x, l-1}^{(N)}$, which has law $\bar{G}_{M}^{(N)}$, where $M$ is equal to the number of stubs with label 3 at the moment we generate $X_{x, l-1}^{(N)}$. This number is bounded from above by $2 M_{l-2}^{(N)}+2 x \leq N^{\alpha}$ by A.4.1(a)) and the fact that $x \leq N^{\frac{(1-\varepsilon / 2)}{\kappa(\tau-1)}} \leq \frac{1}{4} N^{\alpha}$. Indeed, the maximal possible value for $M$ corresponds to the moment we sample $X_{x, l-1}^{(N)}$, i.e., $M$ is at most the number of stubs with label 3 in $S P G_{l-1}$ plus $2 x$ for the pairing at most $x$ free stubs of $S P G_{l-1}$. Hence, due to Lemma A.3.4 $M$ is at most $2 M_{l-2}^{(N)}+2 x \leq N^{\alpha}$, and due to A.3.8 we can take $M=N^{\alpha}$. Therefore, conditionally on $\left\{X_{i, l-1}^{(N)}\right\}_{i=1}^{x-1}$, we can bound $X_{x, l-1}^{(N)}$ from below by $\underline{X}_{x, l-1}^{(N)}$, which has law $\bar{G}_{M}^{(N)}$, and this conditional coupling is equivalent to fact that each component of $\left\{X_{i, l-1}^{(N)}\right\}_{i=1}^{x}$ can be bounded from below by the components of $\left\{\underline{X}_{i, l-1}^{(N)}\right\}_{i=1}^{x}$, where $\left\{\underline{X}_{i, l-1}^{(N)}\right\}_{i=1}^{x}$ are i.i.d. copies with law $\bar{G}_{N^{\alpha}}^{(N)}$.

We finally restrict to $x=Z_{l-1}^{(N)} \wedge N^{\frac{1-\varepsilon / 2}{\kappa(\tau-1)}}$. Note that $y=2\left(x / l^{3}\right)^{\kappa-c_{\gamma} \gamma(x)} \leq N^{\alpha}$, so that $F_{\alpha, N^{\alpha}}$ holds, which in turn implies condition (A.1.30). We can therefore apply Lemma A.1.4(ii) with $\hat{X}_{i}^{(N)}=\underline{X}_{i, l-1}^{(N)}, i=1,2, \ldots, x, \hat{H}^{(N)}=\underline{G}_{N^{\alpha}}^{(N)}$, and $y=2\left(x / l^{3}\right)^{\kappa-c_{\gamma} \gamma(x)}$ to obtain from (A.4.28),

$$
\begin{aligned}
& \mathbb{P}_{N^{\alpha}}\left(\hat{F}_{m, l}(\varepsilon) \cap\left\{\underline{Z}_{x, l}^{(N)} \leq 2\left(\frac{x}{l^{3}}\right)^{\kappa-c_{\gamma} \gamma(x)}\right\}\right) \\
& \quad \leq \mathbb{P}\left(\max _{1 \leq i \leq x} \underline{X}_{i, l-1}^{(N)} \leq y\right) \leq\left(1-\left[1-2 N^{-h}\right][1-G(y)]\right)^{x} .
\end{aligned}
$$

From the lower bound of (A.1.1),

$$
[1-G(y)] \geq y^{-\kappa^{-1}-K_{\tau} \gamma(y)}=2^{\kappa-c_{\gamma} \gamma(x)}\left(x / l^{3}\right)^{\left(-\kappa^{-1}-K_{\tau} \gamma(y)\right)\left(\kappa-c_{\gamma} \gamma(x)\right)} \geq \frac{l^{3}}{x}
$$


because $x / l^{3}>1$ and

$$
\kappa^{-1} c_{\gamma} \gamma(x)-\kappa K_{\tau} \gamma(y)+c_{\gamma} K_{\tau} \gamma(x) \gamma(y) \geq c_{\gamma} \kappa^{-1} \gamma(x)-\kappa K_{\tau} \gamma(y) \geq 0
$$

by choosing $c_{\gamma}$ large and using $\gamma(x) \geq \gamma(y)$. Combining A.4.29 and A.4.30 and taking $1-$ $2 N^{-h}>\frac{1}{2}$, we conclude that

$$
\left(1-\left[1-2 N^{-h}\right][1-G(y)]\right)^{x} \leq\left(1-\frac{l^{3}}{2 x}\right)^{x} \leq e^{-l^{3} / 2} \leq l^{-3},
$$

because $l>m$ and $m$ can be chosen large. This yields A.4.26 with $b=1$.

In the proof of Proposition 3.2, in Section A.5, we often use a corollary of Proposition A.3.2 that we formulate and prove below.

Corollary A.4.2 Let $F$ satisfy Assumption 1.1, For any $\varepsilon>0$ sufficiently small, there exists an integer $m$ such that such that for any $k>m$,

$$
\mathbb{P}\left(\hat{F}_{m, k}(\varepsilon) \cap\left\{\left|Y_{k}^{(N)}-Y_{k-1}^{(N)}\right|>(\tau-2+\varepsilon)^{k(1-\gamma)}\right\}\right) \leq k^{-2},
$$

for sufficiently large $N$.

Proof. We use that part $(a)$ and part $(b)$ of Proposition A.3.2 together imply:

$$
\mathbb{P}\left(\hat{F}_{m, k}(\varepsilon) \cap\left\{\left|\log \left(Z_{k}^{(N)}\right)-\kappa \log \left(Z_{k-1}^{(N)}\right)\right| \geq \kappa \log \left(k^{3}\right)+c_{\gamma} \gamma\left(Z_{k-1}^{(N)}\right) \log \left(k^{3} Z_{k-1}^{(N)}\right)\right\}\right) \leq 2 b k^{-3} .
$$

Indeed applying Proposition A.3.2 with $l=k$ and $x=Z_{k-1}^{(N)}$, and hence $Z_{x, k}=Z_{k}$, yields:

$$
\begin{aligned}
& \mathbb{P}\left(\hat{F}_{m, k}(\varepsilon) \cap\left\{Z_{k}^{(N)} \geq\left(k^{3} x\right)^{\kappa+c_{\gamma} \gamma(x)}\right\}\right) \leq b k^{-3}, \\
& \mathbb{P}\left(\hat{F}_{m, k}(\varepsilon) \cap\left\{Z_{k}^{(N)} \leq\left(x / k^{3}\right)^{\kappa-c_{\gamma} \gamma(x)}\right\}\right) \leq b k^{-3},
\end{aligned}
$$

and from the identities

$$
\begin{aligned}
& \left\{Z_{k}^{(N)} \geq\left(k^{3} x\right)^{\kappa+c_{\gamma} \gamma(x)}\right\}=\left\{\log \left(Z_{k}^{(N)}\right)-\kappa \log \left(Z_{k-1}^{(N)}\right) \geq \log \left(\left(k^{3} x\right)^{\kappa+c_{\gamma} \gamma(x)}\right)-\kappa \log x\right\}, \\
& \left\{Z_{k}^{(N)} \leq\left(x / k^{3}\right)^{\kappa-c_{\gamma} \gamma(x)}\right\}=\left\{\log \left(Z_{k}^{(N)}\right)-\kappa \log \left(Z_{k-1}^{(N)}\right) \leq \log \left(\left(x / k^{3}\right)^{\kappa+c_{\gamma} \gamma(x)}\right)-\kappa \log x\right\},
\end{aligned}
$$

we obtain (A.4.33).

Applying (A.4.33) and (A.3.9), we arrive at

$$
\begin{aligned}
& \mathbb{P}\left(\hat{F}_{m, k}(\varepsilon) \cap\left\{\left|Y_{k}^{(N)}-Y_{k-1}^{(N)}\right|>(\tau-2+\varepsilon)^{k(1-\gamma)}\right\}\right) \\
& \quad \leq \mathbb{P}\left(\hat{F}_{m, k}(\varepsilon) \cap\left\{\kappa^{-k}\left[\kappa \log \left(k^{3}\right)+c_{\gamma} \gamma\left(Z_{k-1}^{(N)}\right) \log \left(k^{3} Z_{k-1}^{(N)}\right)\right]>(\tau-2+\varepsilon)^{k(1-\gamma)}\right\}\right)+2 b k^{-3} .
\end{aligned}
$$

Observe that, due to Lemma A.4.1(c), and since $\gamma(x)=(\log x)^{\gamma-1}$, where $0 \leq \gamma<1$, we have on $\hat{F}_{m, k}(\varepsilon)$,

$$
\begin{aligned}
\kappa^{-k} & {\left[\kappa \log \left(k^{3}\right)+c_{\gamma} \gamma\left(Z_{k-1}^{(N)}\right) \log \left(k^{3} Z_{k-1}^{(N)}\right)\right] } \\
& =\kappa^{-k}\left[\kappa \log \left(k^{3}\right)+c_{\gamma}\left(\log \left(Z_{k-1}^{(N)}\right)\right)^{\gamma-1}\left(\log \left(k^{3}\right)+\log \left(Z_{k-1}^{(N)}\right)\right)\right] \\
& \leq \kappa^{-k}\left[\kappa \log \left(k^{3}\right)+c_{\gamma} \log \left(k^{3}\right)+c_{\gamma}\left(\log \left(Z_{k-1}^{(N)}\right)\right)^{\gamma}\right] \\
& \leq \kappa^{-k}\left[\left(c_{\gamma}+\kappa\right) \log \left(k^{3}\right)+c_{\gamma}\left(\kappa^{k-1}\left(\varepsilon^{-1}+\varepsilon^{3}\right)\right)^{\gamma}\right] \\
& \leq \kappa^{-k(1-\gamma)}\left[\kappa^{-k \gamma}\left(c_{\gamma}+\kappa\right) \log \left(k^{3}\right)+c_{\gamma}\left(\kappa^{-1}\left(\varepsilon^{-1}+\varepsilon^{3}\right)\right)^{\gamma}\right] \leq(\tau-2+\varepsilon)^{k(1-\gamma)},
\end{aligned}
$$


because, for $k$ large, and since $\kappa^{-1}=\tau-2$,

$$
\left(\frac{\tau-2}{\tau-2+\varepsilon}\right)^{k(1-\gamma)}\left[\kappa^{-k \gamma}\left(c_{\gamma}+\kappa\right) \log \left(k^{3}\right)\right] \leq \frac{1}{2}, \quad\left(\frac{\tau-2}{\tau-2+\varepsilon}\right)^{k(1-\gamma)} c_{\gamma}\left(\kappa^{-1}\left(\varepsilon^{-1}+\varepsilon^{3}\right)\right)^{\gamma} \leq \frac{1}{2} .
$$

We conclude that the first term on the right-hand side of (A.4.36) is 0 , for sufficiently large $k$, and the second term is bounded by $2 b k^{-3} \leq k^{-2}$, and hence the statement of the corollary follows.

\section{A.5 Proof of Proposition 3.2 and Proposition 3.3}

Proof of Proposition [3.2(a). We have to show that

$$
\mathbb{P}\left(\varepsilon \leq Y_{m}^{(i, N)} \leq \varepsilon^{-1}, \max _{k \in \mathcal{T}_{m}^{(i, N)}(\varepsilon)}\left|Y_{k}^{(i, N)}-Y_{m}^{(i, N)}\right|>\varepsilon^{3}\right)=o_{N, m, \varepsilon}(1) .
$$

Fix $\varepsilon>0$, such that $\tau-2+\varepsilon<1$. Then, take $m=m_{\varepsilon}$, such that A.3.10 holds, and increase $m$, if necessary, until (A.4.32) holds.

We use the inclusion that (recall the definition of $\mathcal{T}_{m}^{(N)}(\varepsilon)$ given in (3.5)),

$$
\left\{\max _{k \in \mathcal{T}_{m}^{(N)}(\varepsilon)}\left|Y_{k}^{(N)}-Y_{m}^{(N)}\right|>\varepsilon^{3}\right\} \subset\left\{\sum_{k \in \mathcal{T}_{m}^{(N)}(\varepsilon)}\left|Y_{k}^{(N)}-Y_{k-1}^{(N)}\right|>\sum_{k \geq m}(\tau-2+\varepsilon)^{k(1-\gamma)}\right\} .
$$

If the event on the right-hand side of (A.5.1) holds, then there must be a $k \in \mathcal{T}_{m}^{(N)}(\varepsilon)$ such that $\left|Y_{k}^{(N)}-Y_{k-1}^{(N)}\right|>(\tau-2+\varepsilon)^{k(1-\gamma)}$, and therefore

$$
\left\{\max _{k \in \mathcal{T}_{m}^{(N)}(\varepsilon)}\left|Y_{k}^{(N)}-Y_{m}^{(N)}\right|>\varepsilon^{3}\right\} \subset \bigcup_{k \in \mathcal{T}_{m}^{(N)}(\varepsilon)} F_{m, k-1} \cap F_{m, k}^{c}
$$

where we denote

$$
F_{m, k}=F_{m, k}(\varepsilon)=\bigcap_{j=m+1}^{k}\left\{\left|Y_{j}^{(N)}-Y_{j-1}^{(N)}\right| \leq(\tau-2+\varepsilon)^{j(1-\gamma)}\right\} .
$$

Since (A.3.10) implies that on $F_{m, k-1}$ we have $\left|Y_{j}^{(N)}-Y_{m}^{(N)}\right| \leq \varepsilon^{3}, m<j \leq k-1$, we find,

$$
F_{m, k-1} \cap F_{m, k}^{c} \subset\left\{\left|Y_{l}^{(N)}-Y_{m}^{(N)}\right| \leq \varepsilon^{3}, \forall l: m<l \leq k-1\right\} \cap\left\{\left|Y_{k}^{(N)}-Y_{k-1}^{(N)}\right|>(\tau-2+\varepsilon)^{k(1-\gamma)}\right\} .
$$

Take $N$ sufficiently large such that, by Proposition 3.1

$$
\begin{aligned}
\mathbb{P}\left(M_{m}^{(N)}>2 Z_{m}^{(N)}, \varepsilon \leq Y_{m}^{(N)} \leq \varepsilon^{-1}\right) & \leq \mathbb{P}\left(\exists l \leq m: Y_{l}^{(N)} \neq Y_{l}\right)+\mathbb{P}\left(M_{m}>2 \mathcal{Z}_{m}, \varepsilon \leq Y_{m} \leq \varepsilon^{-1}\right) \\
& \leq \mathbb{P}\left(M_{m}>2 \mathcal{Z}_{m}, \varepsilon \leq Y_{m} \leq \varepsilon^{-1}\right)+\varepsilon / 4
\end{aligned}
$$

Next, we use that

$$
\lim _{m \rightarrow \infty} \mathbb{P}\left(M_{m}>2 \mathcal{Z}_{m}, \varepsilon \leq Y_{m} \leq \varepsilon^{-1}\right)=0,
$$

since $Y_{l}=(\tau-2)^{l} \log \mathcal{Z}_{l}$ converges a.s., so that when $Y_{m} \geq \varepsilon$ and $m$ is large, $M_{m-1}$ is much smaller than $Z_{m}$, so that $M_{m}=M_{m-1}+\mathcal{Z}_{m}>2 \mathcal{Z}_{m}$ has small probability, as $m$ is large. 
Then we use (A.5.1 - A.5.6), together with A.3.12, to derive that

$$
\begin{aligned}
& \mathbb{P}\left(\varepsilon \leq Y_{m}^{(N)} \leq \varepsilon^{-1}, \max _{k \in \mathcal{T}_{m}^{(N)}(\varepsilon)}\left|Y_{k}^{(N)}-Y_{m}^{(N)}\right|>\varepsilon^{3}\right) \\
& \quad \leq \mathbb{P}\left(\varepsilon \leq Y_{m}^{(N)} \leq \varepsilon^{-1}, \max _{k \in \mathcal{T}_{m}^{(N)}(\varepsilon)}\left|Y_{k}^{(N)}-Y_{m}^{(N)}\right|>\varepsilon^{3}, Y_{l}^{(N)}=Y_{l}, \forall l \leq m\right)+\mathbb{P}\left(\exists l \leq m: Y_{l}^{(N)} \neq Y_{l}\right) \\
& \quad \leq \sum_{k>m} \mathbb{P}\left(F_{m, k-1} \cap F_{m, k}^{c} \cap\left\{k \in \mathcal{T}_{m}^{(N)}(\varepsilon)\right\} \cap\left\{\varepsilon \leq Y_{m}^{(N)} \leq \varepsilon^{-1}\right\} \cap\left\{Y_{l}^{(N)}=Y_{l}, \forall l \leq m\right\}\right)+\frac{\varepsilon}{2} \\
& \quad \leq \sum_{k>m} \mathbb{P}\left(\hat{F}_{m, k}(\varepsilon) \cap\left\{\left|Y_{k}^{(N)}-Y_{k-1}^{(N)}\right|>(\tau-2+\varepsilon)^{k(1-\gamma)}\right\}\right)+\varepsilon<3 \varepsilon / 2,
\end{aligned}
$$

by Corollary A.4.2

Proof of Proposition [3.2(b). We first show (3.8), then (3.7). Due to Proposition [3.2(a), and using that $\left\{Y_{m}^{(N)} \leq \varepsilon^{-1}\right\}$, we find

$$
Y_{k}^{(N)} \leq Y_{m}^{(N)}+\varepsilon^{3} \leq Y_{m}^{(N)}\left(1+\varepsilon^{2}\right)
$$

apart from an event with probability $o_{N, m, \varepsilon}(1)$, for all $k \in \mathcal{T}_{m}^{(N)}$. By (A.3.9) and because $k \in \mathcal{T}_{m}^{(N)}$, this is equivalent to

$$
Z_{k}^{(N)} \leq\left(Z_{m}^{(N)}\right)^{\kappa^{k-m}\left(1+\varepsilon^{2}\right)} \leq N^{\frac{1-\varepsilon^{2}}{\tau-1}\left(1+\varepsilon^{2}\right)}=N^{\frac{1-\varepsilon^{4}}{\tau-1}} .
$$

We next show (3.7). Observe that $k \in \mathcal{T}_{m}^{(N)}$ implies that either $k-1 \in \mathcal{T}_{m}^{(N)}$, or $k-1=m$. Hence, from $k \in \mathcal{T}_{m}^{(N)}$ and Proposition 3.2 (a), we obtain, apart from an event with probability $o_{N, m, \varepsilon}(1)$,

$$
Y_{k-1}^{(N)} \geq Y_{m}^{(N)}-\varepsilon^{3} \geq \varepsilon-\varepsilon^{3} \geq \frac{\varepsilon}{2},
$$

for $\varepsilon>0$ sufficiently small, and

$$
Y_{k}^{(N)}=Y_{k}^{(N)}-Y_{m}^{(N)}+Y_{m}^{(N)}-Y_{k-1}^{(N)}+Y_{k-1}^{(N)} \geq Y_{k-1}^{(N)}-2 \varepsilon^{3} \geq Y_{k-1}^{(N)}\left(1-4 \varepsilon^{2}\right),
$$

By (A.3.9) this is equivalent to

$$
Z_{k}^{(N)} \geq\left(Z_{k-1}^{(N)}\right)^{\kappa\left(1-4 \varepsilon^{2}\right)} \geq Z_{k-1}^{(N)}
$$

when $\varepsilon>0$ is so small that $\kappa\left(1-4 \varepsilon^{2}\right) \geq 1$, since $\tau \in(2,3)$, and $\kappa=(\tau-2)^{-1}$.

Proof of Proposition [3.3. We must show that

$$
\mathbb{P}\left(k \in \partial \mathcal{T}_{m}^{(N)}(\varepsilon), \varepsilon \leq Y_{m}^{(N)} \leq \varepsilon^{-1}, Z_{k+1}^{(N)} \leq N^{\frac{1-\varepsilon}{\tau-1}}\right)=o_{N, m, \varepsilon}(1),
$$

where

$$
\left\{k \in \partial \mathcal{T}_{m}^{(N)}\right\}=\left\{k \in \mathcal{T}_{m}^{(N)}\right\} \cap\left\{k+1 \notin \mathcal{T}_{m}^{(N)}\right\} .
$$

In the proof, we will make repeated use of Propositions 3.2 and 3.1, whose proofs are now complete. According to the definition of $\hat{F}_{m, k}(\varepsilon)$ in (A.3.12),

$$
\begin{aligned}
& \mathbb{P}\left(\left\{k \in \partial \mathcal{T}_{m}^{(N)}(\varepsilon)\right\} \cap\left\{\varepsilon \leq Y_{m}^{(N)} \leq \varepsilon^{-1}\right\} \cap \hat{F}_{m, k}(\varepsilon)^{c}\right) \\
& \quad \leq \mathbb{P}\left(\varepsilon \leq Y_{m}^{(N)} \leq \varepsilon^{-1}, \max _{l \in \mathcal{T}_{m}^{(N)}(\varepsilon)}\left|Y_{l}^{(N)}-Y_{m}^{(N)}\right|>\varepsilon^{3}\right)+\mathbb{P}\left(\varepsilon \leq Y_{m}^{(N)} \leq \varepsilon^{-1}, M_{m}^{(N)}>2 Z_{m}^{(N)}\right) .
\end{aligned}
$$


In turn Propositions 3.2(a) and 3.1, as well as A.5.5 A.5.6 imply that both probabilities on the right-hand side of (A.5.12) are $o_{\varepsilon}(1)$, as first $N \rightarrow \infty$, and then $m \rightarrow \infty$. Therefore, it suffices to show

$$
\begin{aligned}
& \mathbb{P}\left(\left\{k \in \partial \mathcal{T}_{m}^{(N)}(\varepsilon), \varepsilon \leq Y_{m}^{(N)} \leq \varepsilon^{-1}, Z_{k+1}^{(N)} \leq N^{\frac{1-\varepsilon}{\tau-1}}\right\} \cap \hat{F}_{m, k}(\varepsilon)\right) \\
& \quad=\mathbb{P}\left(\left\{k+1 \notin \mathcal{T}_{m}^{(N)}(\varepsilon), Z_{k+1}^{(N)} \leq N^{\frac{1-\varepsilon}{\tau-1}}\right\} \cap \hat{F}_{m, k}(\varepsilon)\right)=o_{N, m, \varepsilon}(1)
\end{aligned}
$$

Let $x=N^{\frac{1-\varepsilon / 2}{\kappa(\tau-1)}}$, and define the event $I_{N, k}=I_{N, k}(a) \cap I_{N, k}(b) \cap I_{N, k}(c) \cap I_{N, k}(d)$, where

$$
\begin{aligned}
I_{N, k}(a) & =\left\{M_{k-1}^{(N)}<N^{\frac{1-3 \varepsilon^{4} / 4}{\kappa(\tau-1)}}\right\} \\
I_{N, k}(b) & =\left\{x \leq Z_{k}^{(N)}\right\} \\
I_{N, k}(c) & =\left\{Z_{k}^{(N)} \leq N^{\frac{1-\varepsilon^{4}}{\tau-1}}\right\} \\
I_{N, k}(d) & =\left\{Z_{k+1}^{(N)} \geq Z_{x, k+1}^{(N)}-Z_{k}^{(N)}\right\} .
\end{aligned}
$$

We split

$$
\begin{aligned}
\mathbb{P}(\{k+ & \left.\left.1 \notin \mathcal{T}_{m}^{(N)}(\varepsilon), Z_{k+1}^{(N)} \leq N^{\frac{1-\varepsilon}{\tau-1}}\right\} \cap \hat{F}_{m, k}(\varepsilon)\right) \\
= & \mathbb{P}\left(\left\{k+1 \notin \mathcal{T}_{m}^{(N)}(\varepsilon), Z_{k+1}^{(N)} \leq N^{\frac{1-\varepsilon}{\tau-1}}\right\} \cap \hat{F}_{m, k}(\varepsilon) \cap I_{N, k}\right) \\
& +\mathbb{P}\left(\left\{k+1 \notin \mathcal{T}_{m}^{(N)}(\varepsilon), Z_{k+1}^{(N)} \leq N^{\frac{1-\varepsilon}{\tau-1}}\right\} \cap \hat{F}_{m, k}(\varepsilon) \cap I_{N, k}^{c}\right) .
\end{aligned}
$$

We claim that both probabilities are small, which would complete the proof. We start to show that

$$
\mathbb{P}\left(\left\{k+1 \notin \mathcal{T}_{m}^{(N)}(\varepsilon), Z_{k+1}^{(N)} \leq N^{\frac{1-\varepsilon}{\tau-1}}\right\} \cap \hat{F}_{m, k}(\varepsilon) \cap I_{N, k}\right)=o_{N, m, \varepsilon}(1) .
$$

Indeed, by Lemma 5.1 and (3.8),

$$
\begin{aligned}
\mathbb{P}(\{k & \left.\left.\left.+1 \notin \mathcal{T}_{m}^{(N)}(\varepsilon)\right\} \cap Z_{k}^{(N)} \geq N^{\frac{1-\varepsilon}{\tau-1}}\right\} \cap \hat{F}_{m, k}(\varepsilon) \cap I_{N, k}\right) \\
& \leq \mathbb{P}\left(\left\{k \in \mathcal{T}_{m}^{(N)}(\varepsilon)\right\} \cap\left\{\varepsilon \leq Y_{m}^{(N)} \leq \varepsilon^{-1}\right\} \cap\left\{Z_{k}^{(N)} \in\left[N^{\frac{1-\varepsilon}{\tau-1}}, N^{\frac{1-\varepsilon^{4}}{\tau-1}}\right]\right\}\right)+o_{N, m, \varepsilon}(1)=o_{N, m, \varepsilon}(1),
\end{aligned}
$$

where $u=(\tau-1)^{-1}$. Therefore, we are left to deal with the case where $Z_{k}^{(N)} \leq N^{\frac{1-\varepsilon}{\tau-1}}$. For this, and assuming $I_{N, k}$, we can use Proposition A.3.2(b) with $x=N^{\frac{1-\varepsilon / 2}{\kappa(\tau-1)}} \leq Z_{k}^{(N)}$ by $I_{N, k}(b)$, and $l=k+1$ to obtain that, whp,

$$
Z_{k+1}^{(N)} \geq Z_{x, k+1}^{(N)}-Z_{k}^{(N)} \geq x^{\kappa(1-\varepsilon / 2)}-N^{\frac{1-\varepsilon}{\tau-1}}=N^{\frac{(1-\varepsilon / 2)^{2}}{\tau-1}}-N^{\frac{1-\varepsilon}{\tau-1}}>N^{\frac{1-\varepsilon}{\tau-1}}
$$

where we have used that when $k \in \mathcal{T}_{m}^{(N)}(\varepsilon)$ and $Y_{m}^{(N)}>\varepsilon$, then we have $k \leq c \log \log N$, for some $c=c(\tau, \varepsilon)$, and hence, for $N$ large enough,

$$
(k+1)^{3\left(\kappa-c_{\gamma} \gamma(x)\right)} x^{c_{\gamma} \gamma(x)} \leq(k+1)^{3 \kappa} x^{c_{\gamma} \gamma(x)} \leq x^{\varepsilon \kappa / 2} .
$$

This proves A.5.18.

For the second probability on the right-hand side of (A.5.17) it suffices to prove that

$$
\mathbb{P}\left(\left\{k+1 \notin \mathcal{T}_{m}^{(N)}(\varepsilon)\right\} \cap \hat{F}_{m, k}(\varepsilon) \cap I_{N, k}^{c}\right)=o_{N, m, \varepsilon}(1) .
$$

In order to prove (A.5.21), we prove that A.5.21 holds with $I_{N, k}^{c}$ replaced by each one of the four events $I_{N, k}^{c}(a), \ldots, I_{N, k}^{c}(d)$. For the intersection with the event $I_{N, k}^{c}(a)$, we apply Lemma A.4.1(a), which states that $\hat{F}_{m, k}(\varepsilon) \cap I_{N, k}^{c}(a)$ is an empty set. 
It follows from (3.5) that if $k+1 \notin \mathcal{T}_{m}^{(N)}(\varepsilon)$, then

$$
\kappa^{k+1} Y_{m}^{(N)}>\frac{1-\varepsilon^{2}}{\tau-1} \log N
$$

If $\hat{F}_{m, k}(\varepsilon)$ holds then by definition (A.3.12), and Corollary A.4.2 whp,

$$
Y_{k}^{(N)} \geq Y_{k-1}^{(N)} \geq Y_{m}^{(N)}-\varepsilon^{3} \geq Y_{m}^{(N)}\left(1-\varepsilon^{2}\right) .
$$

Hence, if $\hat{F}_{m, k}(\varepsilon)$ holds and $k+1 \notin \mathcal{T}_{m}^{(N)}(\varepsilon)$, then, by A.5.22 A.5.23), whp,

$$
\kappa \log \left(Z_{k}^{(N)}\right)=\kappa^{k+1} Y_{k}^{(N)} \geq\left(1-\varepsilon^{2}\right) \kappa^{k+1} Y_{m}^{(N)} \geq \frac{\left(1-\varepsilon^{2}\right)^{2}}{\tau-1} \log N
$$

so that, whp,

$$
Z_{k}^{(N)} \geq x=N^{\frac{1-\varepsilon / 2}{\kappa(\tau-1)}}
$$

for small enough $\varepsilon>0$ and sufficiently large $N$, i.e., we have

$$
\mathbb{P}\left(\left\{k+1 \notin \mathcal{T}_{m}^{(N)}(\varepsilon)\right\} \cap \hat{F}_{m, k}(\varepsilon) \cap I_{N, k}^{c}(b)\right)=o_{N, m, \varepsilon}(1) .
$$

From Proposition 3.2 (b) it is immediate that

$$
\mathbb{P}\left(\left\{k+1 \notin \mathcal{T}_{m}^{(N)}(\varepsilon)\right\} \cap \hat{F}_{m, k}(\varepsilon) \cap I_{N, k}^{c}(c)\right)=o_{N, m, \varepsilon}(1) .
$$

Finally, recall that $Z_{x, k+1}^{(N)}$ is the number of constructed free stubs at level $k+1$ after pairing of the first $x$ stubs at level $k$. After the pairing of the remaining $Z_{k}^{(N)}-x$ stubs at level $k$ they can 'eat up' at most $Z_{k}^{(N)}-x \leq Z_{k}^{(N)}$ stubs, so that $I_{N, k}(d)$ holds with probability 1 , and hence the event in (A.5.21) intersected with $I_{N, k}^{c}(d)$ has probability equal to 0.

This completes the proof of A.5.21 and hence the Proposition.

\section{Acknowledgement}

We thank Eric Cator for useful discussions on uniform continuity used in the proof of Lemma 5.1. The work of RvdH and DZ was supported in part by Netherlands Organisation for Scientific Research (NWO). The work of all authors was performed in part at the Mittag-Leffler Institute. The work of RvdH was performed in part also at Microsoft Research.

\section{References}

[1] W. Aiello, F. Chung and L. Lu. A random graph model for power law graphs. Experiment. Math. 10(1), 53-66, 2001.

[2] W. Aiello, F. Chung and L. Lu. Random evolution of massive graphs. Handbook of Massive Data Sets, J. Abello, P.M. Pardalos and M.G.C. Resende, eds., Kluwer Academic, Dordrecht, 97-122, 2002.

[3] R. Albert and A.-L. Barabási. Emergence of scaling in random networks. Science, 286, 509-512, 1999.

[4] R. Albert and A.-L. Barabási. Statistical mechanics of complex networks. Rev. Mod. Phys., 74, 47-97, 2002.

[5] N. Alon and J. Spencer. The Probabilistic Method. $2^{\text {nd }}$ Edition. Wiley, New York, 2000.

[6] B. Bollobás. Random Graphs, 2nd edition. Academic Press, New York, 2001. 
[7] B. Bollobás, C. Borgs, J.T. Chayes and O. Riordan. Directed scale-free graphs. Proceedings of the Fourteenth Annual ACM-SIAM Symposium on Discrete Algorithms (Baltimore, MD, 2003), 132-139, ACM, New York, 2003.

[8] B. Bollobás and O. Riordan. The diameter of scale-free random graphs. Preprint Dept. of Math. Sciences, University of Memphis, 2003.

[9] B. Bollobás and O. Riordan. Mathematical results on scale-free random graphs. Handbook of graphs and networks, 1-34, Wiley-VCH, Weinheim, 2003.

[10] B. Bollobás and O. Riordan. Coupling scale-free and classical random graphs. Preprint, 2003.

[11] B. Bollobás, O. Riordan, J. Spencer and G. Tusnády. The degree sequence of a scale-free random graph process. Random Structures Algorithms, 18, 279-290, 2001.

[12] F. Chung and L. Lu. The average distances in random graphs with given expected degrees. PNAS, 99(25), 15879-15882, 2002.

[13] F. Chung and L. Lu. Connected components in random graphs with given expected degree sequences. Annals of Combinatorics, 6, 125-145, 2002.

[14] R. Cohen and S. Havlin. Scale-free networks are ultrasmall. Phys. Rev. Lett., 90, 058701, 1-4, 2003.

[15] C. Cooper and A.A. Frieze. A general model of web graphs. Random Structures Algorithms, $22(3), 311-335,2003$.

[16] P.L. Davies. The simple branching process: A note on convergence when the mean is infinite. J. Appl. Probab. 15, 466-480, 1978.

[17] S.N. Dorogovtsev, A.V. Goltsev and J.F.F. Mendes. Pseudofractal scale-free web. Phys. Rev. E 65, 066122, 2002.

[18] S.N. Dorogovtsev, J.F.F. Mendes and A.N. Samuhkin. Metric structure of random networks. Nucl. Phys. B. 653, 307, 2003.

[19] C. Faloutsos, P. Faloutsos and M. Faloutsos. On power-law relationships of the internet topology. Computer Communications Rev., 29, 251-262, 1999.

[20] W. Feller. An Introduction to Probability Theory and Its Applications. Volume II, 2nd edition, Wiley, New York, 1971.

[21] R. van der Hofstad, G. Hooghiemstra and P. Van Mieghem. Random graphs with finite variance degrees. Preprint 2003, accepted for publication in Random Structures Algorithms.

[22] R. van der Hofstad, G. Hooghiemstra and D. Znamenski. Distances in random graphs with infinite mean degrees. Preprint, submitted to Extremes, 2004.

[23] R. van der Hofstad, G. Hooghiemstra and D. Znamenski. Random graphs with arbitrary i.i.d. degrees. Preprint Mittag-Leffler Institute, 2004.

[24] S. Janson. On concentration of probability. Contemporary Combinatorics, ed. B. Bollobás, Bolyai Soc. Math. Stud. 10, 289-301, János Bolyai Mathematical Society, Budapest, 2002.

[25] S. Janson, T. Luczak and A. Rucinski. Random Graphs. Wiley, New York, 2000.

[26] R. Kumar, P. Raghavan, S. Rajagopalan, D. Sivakumar, A. Tomkins and E. Upfal. Stochastic Models for the Web Graph, 42st Annual IEEE Symposium on Foundations of Computer Science, 57-65, 2000. 
[27] M. Molloy and B. Reed. A critical point for random graphs with a given degree sequence, Random Structures Algorithms, 6, 161-179, 1995.

[28] M. Molloy and B. Reed. The size of the giant component of a random graph with a given degree sequence, Combin. Probab. Comput., 7, 295-305, 1998.

[29] M.E.J. Newman. The structure and function of complex networks. SIAM Rev. 45(2), 167-256, 2003.

[30] M.E.J. Newman, S.H. Strogatz, and D.J. Watts. Random graphs with arbitrary degree distribution and their application, Phys. Rev. E, 64, 026118, 2001.

[31] I. Norros and H. Reittu. On a conditionally Poissonean graph process. Preprint 2004.

[32] H. Reittu and I. Norros. On the power law random graph model of massive data networks, Performance Evalution, 55 (1-2), 3-23, 2004.

[33] H. -J. Schuh and A. D. Barbour. On the asymptotic behaviour of branching processes with infinite mean, Adv. Appl. Probab., 9, 681-723, 1977.

[34] E. Seneta. The simple branching processes with infinite mean. J. Appl. Prob., 10, 206-212, 1973.

[35] H Tangmunarunkit, R. Govindan, S. Jamin, S. Shenker, and W. Willinger. Network topology generators: Degree-based vs. structural. ACM Sigcomm'02, Pittsburgh, Pennsylvania,USA, 19-23, 2002.

[36] D. J. Watts. Small Worlds, The Dynamics of Networks between Order and Randomness. Princeton University Press, Princeton, New Jersey, 1999.

Corresponding author:

G. Hooghiemstra

EEMCS, Delft University of Technology

P.O.Box 5031

2600GA Delft

The Netherlands

E-mail: G.Hooghiemstra@ewi.tudelft.nl 